\title{
Microwave-Assisted Electrostatically Enhanced Phenol-Catalyzed Synthesis of Oxazolidinones
}

Ali Rostami, ${ }^{*}{ }^{[a]}$ Amirhossein Ebrahimi, ${ }^{[a]}$ Nader Sakhaee, ${ }^{[b]}$, Farhad Golmohammadi, ${ }^{[a]}$ Ahmed Al-Harrasi ${ }^{*}[a]$

[a] Natural and Medical Sciences Research Center (NMSRC), University of Nizwa, 616, Nizwa, Sultanate of Oman, Email: arostami@unizwa.edu.om, aharrasi@unizwa.edu.om

${ }^{[b]}$ Roger Adams Laboratory, University of Illinois, 600 South Mathews Avenue, Urbana, Illinois 61801, United States

\section{Table of Contents}

I. ${ }^{1} \mathrm{H}$ NMR analysis for the conversion of a model reaction $(\mathbf{1 b}, \mathbf{2 a}$, and $\mathbf{6 a}$ )------------------S2

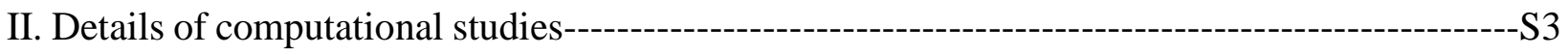

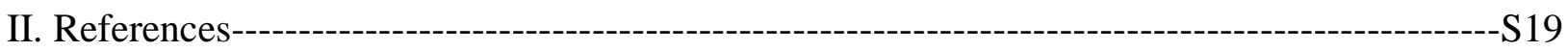

IV. ${ }^{1} \mathrm{H}$ NMR, ${ }^{13} \mathrm{C}$ NMR selected spectra and HPLC traces---------------------------------S20 


\section{I. $\quad{ }^{1} \mathrm{H}$ NMR analysis for the conversion of a model reaction (1b, 2a, and 6a)}<smiles>O=C(Nc1ccccc1)Nc1ccc(F)cc1</smiles>

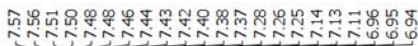
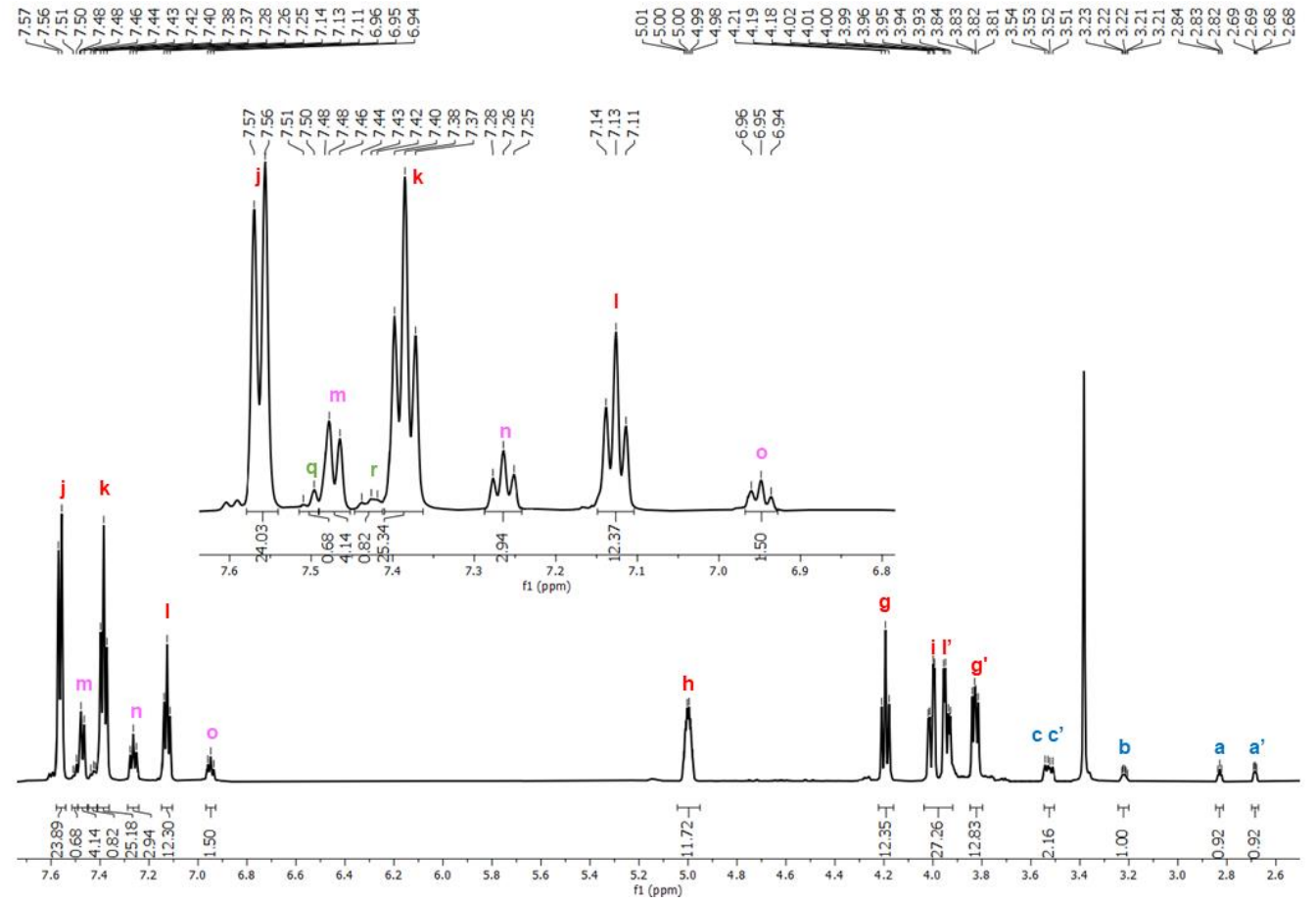

Figure S1. Conditions: epoxide $1 \mathbf{b}(5.0 \mathrm{mmol})$, isocyanate $2(5.0 \mathrm{mmol}), \mathbf{6 a}(5 \mathrm{~mol} \%), 100{ }^{\circ} \mathrm{C}, 20 \mathrm{mins}$, reaction performed as neat; The selectivity was assessed by ${ }^{1} \mathrm{H}$ NMR spectroscopy $\left(\mathrm{CDCl}_{3}\right)$ of the crude reaction mixture. 


\section{Details of computational studies}

All the calculations were performed with the M06-2X/def2-TZVP level using Gaussian g16 package $^{1}$. The thermal corrections as well as zero points are listed along with relative and absolute energies in table S1. In order to assess the minimum energy conformer of $\mathbf{6 a}$, the Spartan software (Spartan 18. Version 14.5$)^{2}$ was employed and the most stable conformation was utilized for the DFT calculations. The propylene oxide and phenyl isocyanate in the presence of catalyst $6 \mathbf{6}$ was used as a model for the calculation. Absolute Energies of isolated reactants in comparison to the energies of complexed reactants were used to define relative energies. Geometry coordinates for all the transition states and intermediates along the reaction coordinate are given. The frequency calculations were carried out for all optimized structures to ensure the absence of any imaginary for the ground state molecules. The intrinsic reaction coordinate (IRC) were carried out in addition to the presence of one imaginary frequency, for the transition state structure. 


\section{i) Conformational screening of $6 \mathrm{a}$}
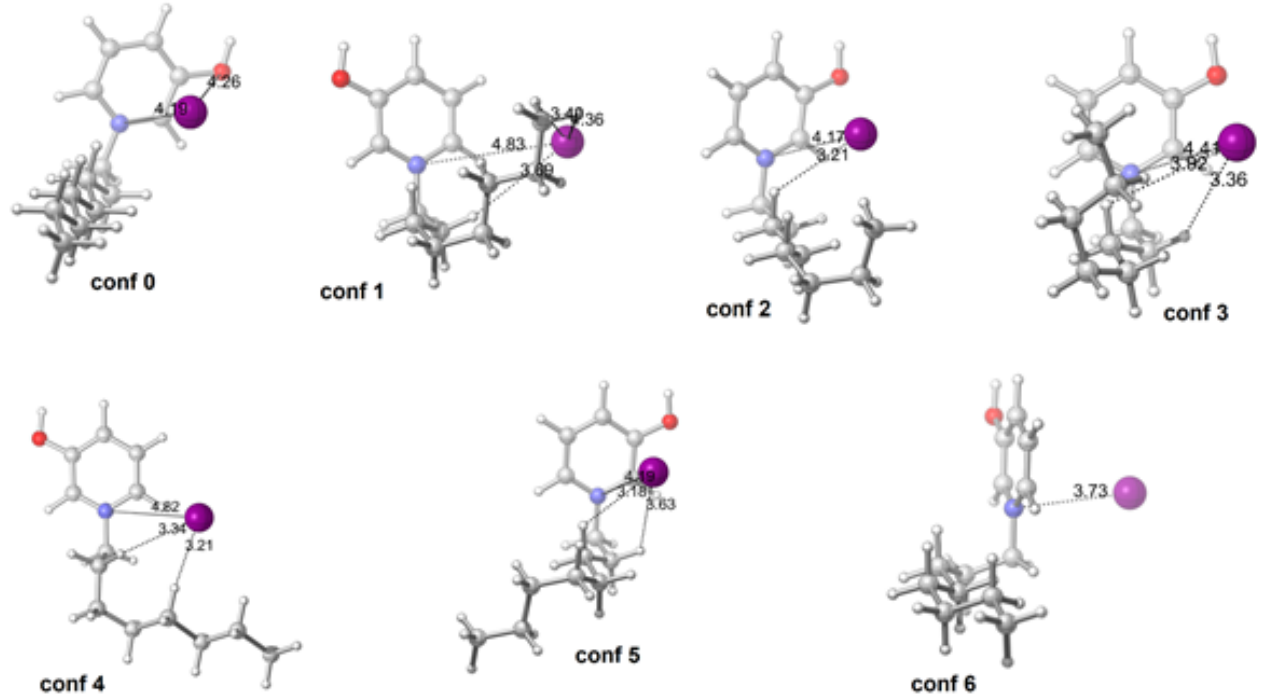

\begin{tabular}{cc} 
Conformer & E rel (kcal) \\
Conf 0 & 0.0 \\
Conf 1 & 3.1 \\
Conf 2 & 3.3 \\
Conf 3 & 4.0 \\
Conf 4 & 4.3 \\
Conf 5 & 4.7 \\
Conf 6 & 5.8 \\
\hline
\end{tabular}

Figure S2. Conformational screening of 6a (Spartan 18. Version 14.5, semi empirical, RM1) 


\section{(ii) Energies of all TS and intermediates:}

$\begin{array}{lccccc}\text { compound } & \begin{array}{c}\text { Absolute } \\ \text { energies (Hartree) }\end{array} & \begin{array}{c}\text { Relative } \\ \text { energies }(\mathrm{kcal})\end{array} & \text { ZPE (Hartree ) } & \text { TC (Hartree ) } & \boldsymbol{i} \text {-frequency }\left(\mathrm{cm}^{-1}\right) \\ \text { TS1-B } & -1750.622327 & 3.81 & 0.298564823 & 0.016320949 & 1092 \\ \text { INT2-B } & -1750.666582 & -23.96 & 0.300000762 & 0.015848651 & - \\ \text { INT1-A } & -1750.64765 & -12.08 & 0.380200041 & 0.018244422 & - \\ \text { TS2-B } & -1750.594184 & 21.47 & 0.32002392 & 0.021120108 & 1308 \\ \text { TS1-A } & -1750.609467 & 11.88 & 0.401563916 & 0.022952168 & 1218 \\ \text { INT2-A } & -1750.662726 & -21.54 & 0.408381769 & 0.021409582 & - \\ \text { Isolated reactants } & -1750.628399 & 0 & - & - & - \\ \text { Isolated products } & -1750.665865 & -23.51 & - & - & - \\ \text { TS2-A } & -1750.637929 & -5.98 & 0.298919047 & 0.016549481 & 815\end{array}$

Table S1: energies of all TS and intermediates, along with zero point energies and thermal corrections. 
(iii) Selected geometries from the main path (as discussed in the manuscript).
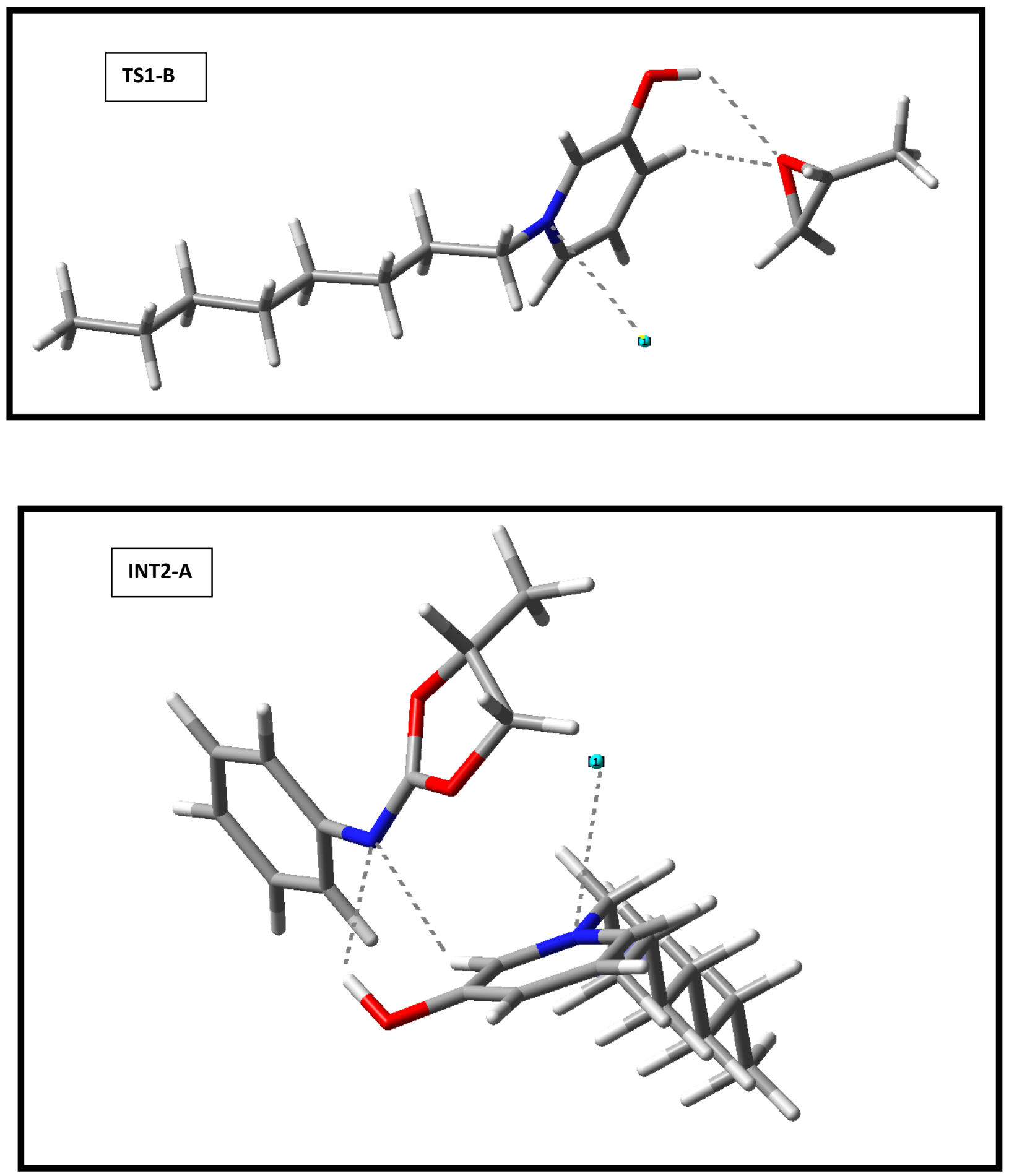

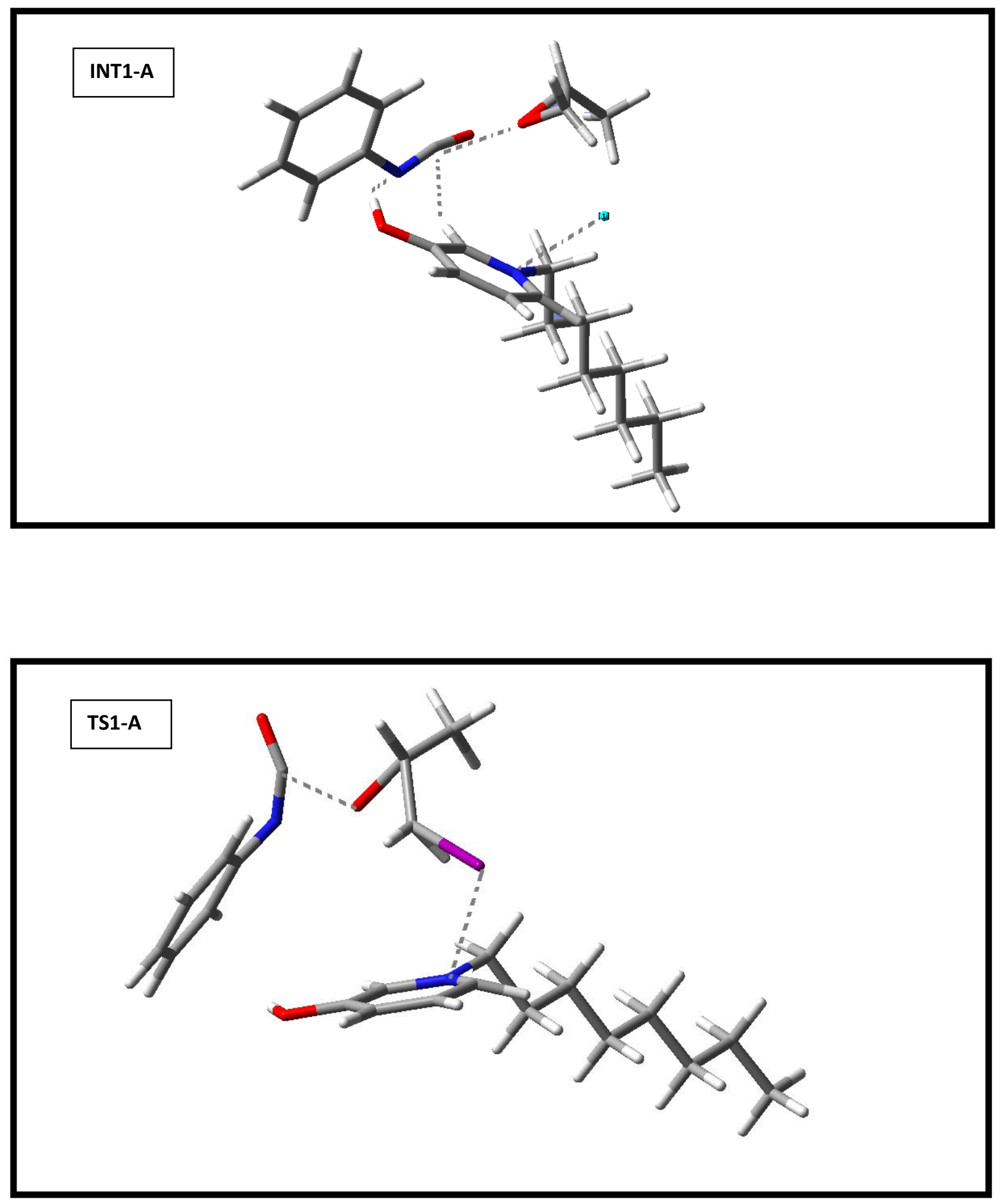

S7 
iv) Geometry coordinates for all TS and intermediates.

TS1-B:

$\begin{array}{lrrr}\mathrm{H} & 1.794664 & 1.151466 & -2.250025 \\ \mathrm{C} & 0.920315 & 0.915955 & -1.632477 \\ \mathrm{C} & -1.212039 & 0.182804 & -0.000185 \\ \mathrm{C} & 0.666426 & -0.453130 & -1.344755 \\ \mathrm{C} & 0.134633 & 1.897241 & -1.098721 \\ \mathrm{C} & -0.955229 & 1.511884 & -0.272833 \\ \mathrm{~N} & -0.402220 & -0.790692 & -0.564355 \\ \mathrm{H} & 1.217310 & -1.253483 & -1.871433 \\ \mathrm{H} & 0.340801 & 2.957796 & -1.282292 \\ \mathrm{C} & -0.583323 & -2.219786 & -0.181140 \\ \mathrm{H} & 0.417219 & -2.627513 & 0.120755 \\ \mathrm{H} & -1.208600 & -2.294663 & 0.738671 \\ \mathrm{C} & -1.207987 & -3.003456 & -1.339607 \\ \mathrm{H} & -0.586386 & -2.924652 & -2.256445 \\ \mathrm{H} & -2.189945 & -2.569765 & -1.624448 \\ \mathrm{C} & -1.366834 & -4.467578 & -0.935061 \\ \mathrm{H} & -0.385961 & -4.900852 & -0.644589 \\ \mathrm{H} & -1.995675 & -4.555751 & -0.023727 \\ \mathrm{C} & -1.978296 & -5.268678 & -2.072610 \\ \mathrm{H} & -1.355234 & -5.251335 & -2.978485 \\ \mathrm{H} & -2.971261 & -4.894975 & -2.361365 \\ \mathrm{O} & -1.837590 & 2.402518 & 0.238589 \\ \mathrm{I} & 2.543099 & -0.644296 & 0.435491 \\ \mathrm{C} & 2.241872 & 3.101098 & 1.486220 \\ \mathrm{H} & 2.371708 & 2.012609 & 1.350140\end{array}$




$\begin{array}{lrrr}\mathrm{H} & 3.108160 & 3.652627 & 1.094629 \\ \mathrm{C} & 1.368484 & 3.673216 & 2.562328 \\ \mathrm{H} & 0.812612 & 3.002411 & 3.246408 \\ \mathrm{O} & 0.957944 & 3.647966 & 1.200925 \\ \mathrm{C} & 1.655315 & 5.036810 & 3.124996 \\ \mathrm{H} & 0.734362 & 5.542196 & 3.449179 \\ \mathrm{H} & 2.147240 & 5.706780 & 2.405140 \\ \mathrm{H} & 2.315083 & 4.960771 & 4.000960 \\ \mathrm{H} & -2.046136 & -0.127879 & 0.644907 \\ \mathrm{H} & -1.357630 & 3.218896 & 0.435834 \\ \mathrm{C} & -2.157307 & -6.741282 & -1.693991 \\ \mathrm{H} & -1.176382 & -7.191131 & -1.502576 \\ \mathrm{H} & -2.774947 & -6.813989 & -0.791522 \\ \mathrm{C} & -2.843789 & -7.483930 & -2.843415 \\ \mathrm{H} & -2.226150 & -7.411224 & -3.745884 \\ \mathrm{H} & -3.824714 & -7.034082 & -3.034830 \\ \mathrm{C} & -3.022801 & -8.956534 & -2.464795 \\ \mathrm{H} & -2.041877 & -9.406383 & -2.273380 \\ \mathrm{H} & -3.640441 & -9.029240 & -1.562326 \\ \mathrm{C} & -3.709284 & -9.699182 & -3.614219 \\ \mathrm{H} & -3.091645 & -9.626477 & -4.516688 \\ \mathrm{H} & -4.690209 & -9.249333 & -3.805633 \\ \mathrm{H} & -3.837435 & -10.753378 & -3.343175\end{array}$


TS1-A:

$\begin{array}{rrrr}\mathrm{C} & 1.200559 & -3.013533 & -2.829777 \\ \mathrm{C} & 1.321912 & -1.775961 & -3.389917 \\ \mathrm{C} & 1.221021 & -0.639566 & -2.537462 \\ \mathrm{C} & 0.954699 & -3.158678 & -1.434630 \\ \mathrm{C} & 0.995729 & -0.776219 & -1.184042 \\ \mathrm{O} & 1.352871 & 0.571842 & -3.164705 \\ \mathrm{H} & 1.205719 & 1.264387 & -2.497161 \\ \mathrm{O} & -4.795481 & 2.891272 & 0.703414 \\ \mathrm{~N} & 0.876007 & -2.049885 & -0.645552 \\ \mathrm{H} & 0.940370 & 0.070563 & -0.509415 \\ \mathrm{O} & -2.884090 & 0.129960 & -0.238302 \\ \mathrm{C} & -2.455053 & -1.517536 & -0.801997 \\ \mathrm{C} & -3.687304 & 2.491350 & 0.718859 \\ \mathrm{C} & 0.505016 & -2.213050 & 0.789051 \\ \mathrm{H} & 0.194867 & -1.276689 & 1.246061 \\ \mathrm{H} & -0.294277 & -2.947635 & 0.920074 \\ \mathrm{C} & 1.735486 & -2.614269 & 1.608025 \\ \mathrm{H} & 2.511431 & -1.843676 & 1.521131 \\ \mathrm{H} & 2.163226 & -3.545088 & 1.217211 \\ \mathrm{C} & 1.333964 & -2.801913 & 3.069421 \\ \mathrm{H} & 0.905429 & -1.870743 & 3.460032 \\ \mathrm{H} & 0.557323 & -3.572942 & 3.144349 \\ \mathrm{C} & 2.539547 & -3.202487 & 3.903602 \\ \mathrm{H} & 2.967477 & -4.137610 & 3.522919 \\ \mathrm{H} & 3.318061 & -2.433307 & 3.834424 \\ \mathrm{C} & -3.859411 & -1.203797 & -0.381489 \\ \mathrm{H} & -4.632415 & -1.119486 & -1.132583 \\ \mathrm{C} & -4.364215 & -1.661627 & 0.957356 \\ \mathrm{H} & -3.563158 & -1.697349 & 1.702773\end{array}$




$\begin{array}{lrrr}\mathrm{H} & -4.797730 & -2.662290 & 0.869135 \\ \mathrm{H} & -5.139339 & -0.978477 & 1.316951 \\ \mathrm{~N} & -2.596383 & 2.868529 & 0.944656 \\ \mathrm{C} & -1.834064 & 3.247371 & -0.182151 \\ \mathrm{C} & -0.132369 & 4.059592 & -2.284650 \\ \mathrm{C} & -0.793418 & 4.160728 & 0.037199 \\ \mathrm{C} & -1.992979 & 2.738807 & -1.484185 \\ \mathrm{C} & -1.151653 & 3.142908 & -2.526190 \\ \mathrm{C} & 0.048701 & 4.568598 & -1.001764 \\ \mathrm{H} & -0.637394 & 4.561566 & 1.036691 \\ \mathrm{H} & -2.769035 & 2.009430 & -1.709082 \\ \mathrm{H} & -1.296199 & 2.737661 & -3.525554 \\ \mathrm{H} & 0.842340 & 5.285406 & -0.806002 \\ \mathrm{H} & 0.516744 & 4.379559 & -3.096376 \\ \mathrm{H} & 1.502655 & -1.641000 & -4.449528 \\ \mathrm{H} & 1.288941 & -3.892118 & -3.458181 \\ \mathrm{H} & 1.263040 & -4.104872 & -0.978587 \\ \mathrm{I} & -1.573171 & -3.652329 & -1.733409 \\ \mathrm{H} & -2.169163 & -1.529279 & -1.841451 \\ \mathrm{H} & -1.695622 & -1.866469 & -0.133713 \\ \mathrm{C} & 2.124749 & -3.385530 & 5.372747 \\ \mathrm{H} & 1.343911 & -4.152943 & 5.440442 \\ \mathrm{H} & 1.694327 & -2.450405 & 5.751552 \\ \mathrm{C} & 3.315519 & -3.789893 & 6.244642 \\ \mathrm{H} & 4.096870 & -3.023140 & 6.177124 \\ \mathrm{H} & 3.746612 & -4.724773 & 5.866306 \\ \mathrm{C} & 2.899428 & -3.970849 & 7.704245 \\ \mathrm{H} & 2.120954 & -4.739199 & 7.777158 \\ \mathrm{H} & 2.471220 & -3.037528 & 8.087977 \\ \mathrm{C} & 4.079718 & -4.372708 & 8.574264\end{array}$




$\begin{array}{llll}\mathrm{H} & 4.863693 & -3.609112 & 8.548970 \\ \mathrm{H} & 4.511485 & -5.320221 & 8.236428 \\ \mathrm{H} & 3.759673 & -4.496662 & 9.613551\end{array}$

INT2-B:

$\begin{array}{lrrr}\mathrm{H} & -4.686858 & -1.912963 & -1.345276 \\ \mathrm{C} & -3.722696 & -1.404496 & -1.240328 \\ \mathrm{H} & -4.547931 & 0.568839 & -1.041807 \\ \mathrm{C} & -3.650750 & -0.054989 & -1.072705 \\ \mathrm{~N} & -1.339849 & -1.598966 & -1.166695 \\ \mathrm{C} & -2.371493 & 0.591099 & -0.928777 \\ \mathrm{C} & -2.555975 & -2.195778 & -1.282413 \\ \mathrm{C} & -1.207082 & -0.271592 & -0.989095 \\ \mathrm{O} & -2.245809 & 1.815067 & -0.757035 \\ \mathrm{H} & -2.611091 & -3.281501 & -1.410476 \\ \mathrm{O} & 0.210870 & 2.741228 & -0.405033 \\ \mathrm{C} & 0.280632 & 3.843084 & 0.431396 \\ \mathrm{C} & -0.220013 & 3.491050 & 1.826069 \\ \mathrm{H} & -0.185702 & 4.340538 & 2.523955 \\ \mathrm{H} & 1.373728 & 4.094507 & 0.465406 \\ \mathrm{C} & -0.119985 & -2.462360 & -1.194005 \\ \mathrm{H} & 0.769793 & -1.860769 & -1.495209 \\ \mathrm{H} & -0.229976 & -3.231412 & -1.993997 \\ \mathrm{C} & 0.084651 & -3.105137 & 0.182183 \\ \mathrm{H} & -0.812655 & -3.687100 & 0.482164 \\ \mathrm{H} & 0.190786 & -2.323602 & 0.965099\end{array}$




$\begin{array}{cccc}\text { C } & 1.319289 & -4.002546 & 0.151186 \\ \text { H } & 2.218714 & -3.420388 & -0.141759 \\ \text { H } & 1.218046 & -4.781673 & -0.633929 \\ \text { C } & 1.535625 & -4.651702 & 1.508426 \\ \text { H } & 0.685447 & -5.279404 & 1.812194 \\ \text { H } & 1.685402 & -3.910510 & 2.307108 \\ \text { H } & -0.207136 & 0.173335 & -0.884042 \\ \text { H } & -1.319739 & 2.401639 & -0.702900 \\ \text { I } & 1.043775 & 2.027717 & 2.545730 \\ \text { H } & -1.245829 & 3.090293 & 1.836722 \\ \text { C } & -0.524255 & 5.028084 & -0.113951 \\ \text { H } & -0.225281 & 5.272396 & -1.142912 \\ \text { H } & -0.376004 & 5.933895 & 0.489089 \\ \text { H } & -1.605147 & 4.826984 & -0.139499 \\ \text { C } & 2.783194 & -5.549344 & 1.469882 \\ \text { H } & 3.658703 & -4.949412 & 1.192685 \\ \text { H } & 2.658374 & -6.318398 & 0.697713 \\ \text { C } & 3.032256 & -6.220275 & 2.822474 \\ \text { H } & 2.157912 & -6.820799 & 3.101541 \\ \text { H } & 3.157450 & -5.452851 & 3.596022 \\ \text { C } & 4.273130 & -7.111784 & 2.780471 \\ \text { H } & 5.151093 & -6.515523 & 2.505961 \\ \text { H } & 4.151530 & -7.883494 & 2.011460 \\ \text { C } & 4.524333 & -7.780485 & 4.122576 \\ \text { H } & 5.416009 & -8.412982 & 4.069905 \\ \text { H } & 3.677263 & -8.410835 & 4.411483 \\ \text { H } & 4.682365 & -7.035279 & 4.908725\end{array}$


INT1-A:

$\begin{array}{rrrr}\mathrm{O} & 0.333079 & 1.918374 & -2.182739 \\ \mathrm{C} & -0.708191 & 1.650578 & -1.359756 \\ \mathrm{O} & 2.050380 & 2.955314 & 1.172500 \\ \mathrm{C} & 2.336377 & 1.830882 & 0.482071 \\ \mathrm{C} & 3.004693 & -0.459049 & -0.900853 \\ \mathrm{C} & 3.363304 & 1.892971 & -0.471363 \\ \mathrm{C} & 1.696894 & 0.620219 & 0.768215 \\ \mathrm{~N} & 2.038175 & -0.500605 & 0.061907 \\ \mathrm{C} & 3.697976 & 0.732772 & -1.146056 \\ \mathrm{H} & 3.867312 & 2.843582 & -0.679044 \\ \mathrm{H} & 4.480474 & 0.737580 & -1.912475 \\ \mathrm{H} & 3.227434 & -1.374415 & -1.475821 \\ \mathrm{O} & -1.752565 & 1.098772 & -2.030511 \\ \mathrm{C} & -1.390518 & 0.870798 & -3.391806 \\ \mathrm{H} & -1.905000 & 1.677507 & -3.982077 \\ \mathrm{C} & 1.267618 & -1.761884 & 0.309821 \\ \mathrm{H} & 1.460595 & -2.504267 & -0.502315 \\ \mathrm{H} & 0.176194 & -1.536965 & 0.222107 \\ \mathrm{C} & 1.642144 & -2.328089 & 1.684467 \\ \mathrm{H} & 1.438832 & -1.597270 & 2.495249 \\ \mathrm{H} & 2.733279 & -2.525130 & 1.747848 \\ \mathrm{C} & 0.855123 & -3.614334 & 1.928974 \\ \mathrm{H} & 1.063078 & -4.358213 & 1.130674 \\ \mathrm{H} & -0.236782 & -3.422864 & 1.858013 \\ \mathrm{C} & 1.199196 & -4.196196 & 3.290140 \\ \mathrm{H} & 0.955193 & -3.509046 & 4.113162 \\ \mathrm{H} & 2.267409 & -4.439152 & 3.383992 \\ \mathrm{H} & 0.880072 & 0.561021 & 1.515165\end{array}$




$\begin{array}{lrrr}\mathrm{H} & 1.086256 & 3.082566 & 1.146497 \\ \mathrm{~N} & -0.636156 & 1.964883 & -0.087491 \\ \mathrm{C} & -1.796766 & 1.822863 & 0.720440 \\ \mathrm{C} & -3.919318 & 1.690763 & 2.521277 \\ \mathrm{C} & -1.594690 & 1.321265 & 2.013105 \\ \mathrm{C} & -3.070018 & 2.255746 & 0.335614 \\ \mathrm{C} & -4.121538 & 2.185281 & 1.238418 \\ \mathrm{C} & -2.654850 & 1.261193 & 2.904660 \\ \mathrm{H} & -0.600200 & 0.974493 & 2.320512 \\ \mathrm{H} & -3.244893 & 2.652743 & -0.668524 \\ \mathrm{H} & -5.117897 & 2.521864 & 0.935965 \\ \mathrm{H} & -2.494605 & 0.871219 & 3.914044 \\ \mathrm{H} & -4.752730 & 1.638899 & 3.226532 \\ \mathrm{C} & -1.904660 & -0.483145 & -3.839061 \\ \mathrm{H} & -2.002729 & -1.214348 & -3.023005 \\ \mathrm{H} & -1.244524 & -0.930209 & -4.595609 \\ \mathrm{H} & -2.906208 & -0.400375 & -4.289676 \\ \mathrm{C} & 0.152358 & 1.135136 & -3.388517 \\ \mathrm{I} & 0.653666 & -0.726458 & -2.494770 \\ \mathrm{H} & 0.997671 & 0.631822 & -3.901652 \\ \mathrm{H} & 0.230627 & 2.014865 & -4.125336 \\ \mathrm{C} & 0.407476 & -5.493645 & 3.522380 \\ \mathrm{H} & 0.644935 & -6.215259 & 2.731077 \\ \mathrm{H} & -0.667405 & -5.284010 & 3.459333 \\ \mathrm{C} & 0.728008 & -6.108739 & 4.886428 \\ \mathrm{H} & 1.802471 & -6.319162 & 4.951505 \\ \mathrm{H} & 0.491102 & -5.388771 & 5.679212 \\ \mathrm{C} & -0.060693 & -7.398157 & 5.113739 \\ \mathrm{H} & 0.175571 & -8.123114 & 4.326136 \\ \mathrm{H} & -1.135818 & -7.192682 & 5.053845\end{array}$




$\begin{array}{lrrr}\mathrm{C} & 0.254801 & -8.012749 & 6.468084 \\ \mathrm{H} & 1.318114 & -8.260205 & 6.548506 \\ \mathrm{H} & -0.319861 & -8.933421 & 6.609642 \\ \mathrm{H} & -0.000546 & -7.324619 & 7.280249\end{array}$

INT2-A:

$\begin{array}{rrrr}\mathrm{O} & 0.333079 & 1.918374 & -2.182739 \\ \mathrm{C} & -0.708191 & 1.650578 & -1.359756 \\ \mathrm{O} & 2.050380 & 2.955314 & 1.172500 \\ \mathrm{C} & 2.336377 & 1.830882 & 0.482071 \\ \mathrm{C} & 3.004693 & -0.459049 & -0.900853 \\ \mathrm{C} & 3.363304 & 1.892971 & -0.471363 \\ \mathrm{C} & 1.696894 & 0.620219 & 0.768215 \\ \mathrm{~N} & 2.038175 & -0.500605 & 0.061907 \\ \mathrm{C} & 3.697976 & 0.732772 & -1.146056 \\ \mathrm{H} & 3.867312 & 2.843582 & -0.679044 \\ \mathrm{H} & 4.480474 & 0.737580 & -1.912475 \\ \mathrm{H} & 3.227434 & -1.374415 & -1.475821 \\ \mathrm{O} & -1.752565 & 1.098772 & -2.030511 \\ \mathrm{C} & -1.390518 & 0.870798 & -3.391806 \\ \mathrm{H} & -1.905000 & 1.677507 & -3.982077 \\ \mathrm{C} & 1.267618 & -1.761884 & 0.309821 \\ \mathrm{H} & 1.460595 & -2.504267 & -0.502315 \\ \mathrm{H} & 0.176194 & -1.536965 & 0.222107 \\ \mathrm{C} & 1.642144 & -2.328089 & 1.684467 \\ \mathrm{H} & 1.438832 & -1.597270 & 2.495249 \\ \mathrm{H} & 2.733279 & -2.525130 & 1.747848 \\ \mathrm{C} & 0.855123 & -3.614334 & 1.928974\end{array}$




$\begin{array}{lrrr}\mathrm{H} & 1.063078 & -4.358213 & 1.130674 \\ \mathrm{H} & -0.236782 & -3.422864 & 1.858013 \\ \mathrm{C} & 1.199196 & -4.196196 & 3.290140 \\ \mathrm{H} & 0.955193 & -3.509046 & 4.113162 \\ \mathrm{H} & 2.267409 & -4.439152 & 3.383992 \\ \mathrm{H} & 0.880072 & 0.561021 & 1.515165 \\ \mathrm{H} & 1.086256 & 3.082566 & 1.146497 \\ \mathrm{~N} & -0.636156 & 1.964883 & -0.087491 \\ \mathrm{C} & -1.796766 & 1.822863 & 0.720440 \\ \mathrm{C} & -3.919318 & 1.690763 & 2.521277 \\ \mathrm{C} & -1.594690 & 1.321265 & 2.013105 \\ \mathrm{C} & -3.070018 & 2.255746 & 0.335614 \\ \mathrm{C} & -4.121538 & 2.185281 & 1.238418 \\ \mathrm{C} & -2.654850 & 1.261193 & 2.904660 \\ \mathrm{H} & -0.600200 & 0.974493 & 2.320512 \\ \mathrm{H} & -3.244893 & 2.652743 & -0.668524 \\ \mathrm{H} & -5.117897 & 2.521864 & 0.935965 \\ \mathrm{H} & -2.494605 & 0.871219 & 3.914044 \\ \mathrm{H} & -4.752730 & 1.638899 & 3.226532 \\ \mathrm{C} & -1.904660 & -0.483145 & -3.839061 \\ \mathrm{H} & -2.002729 & -1.214348 & -3.023005 \\ \mathrm{H} & -1.244524 & -0.930209 & -4.595609 \\ \mathrm{H} & -2.906208 & -0.400375 & -4.289676 \\ \mathrm{C} & 0.152358 & 1.135136 & -3.388517 \\ \mathrm{I} & 0.653666 & -0.726458 & -2.494770 \\ \mathrm{H} & 0.997671 & 0.631822 & -3.901652 \\ \mathrm{H} & 0.230627 & 2.014865 & -4.125336 \\ \mathrm{C} & 0.407476 & -5.493645 & 3.522380 \\ \mathrm{H} & 0.644935 & -6.215259 & 2.731077 \\ \mathrm{H} & -0.667405 & -5.284010 & 3.459333\end{array}$




$\begin{array}{lrrr}\mathrm{C} & 0.728008 & -6.108739 & 4.886428 \\ \mathrm{H} & 1.802471 & -6.319162 & 4.951505 \\ \mathrm{H} & 0.491102 & -5.388771 & 5.679212 \\ \mathrm{C} & -0.060693 & -7.398157 & 5.113739 \\ \mathrm{H} & 0.175571 & -8.123114 & 4.326136 \\ \mathrm{H} & -1.135818 & -7.192682 & 5.053845 \\ \mathrm{C} & 0.254801 & -8.012749 & 6.468084 \\ \mathrm{H} & 1.318114 & -8.260205 & 6.548506 \\ \mathrm{H} & -0.319861 & -8.933421 & 6.609642 \\ \mathrm{H} & -0.000546 & -7.324619 & 7.280249\end{array}$




\section{References}

(1) Gaussian 16, Revision C.01, Frisch, M. J.; Trucks, G. W.; Schlegel, H. B.; Scuseria, G. E.; Robb, M. A.; Cheeseman, J. R.; Scalmani, G.; Barone, V.; Petersson, G. A.; Nakatsuji, H.; Li, X.; Caricato, M.; Marenich, A. V.; Bloino, J.; Janesko, B. G.; Gomperts, R.; Mennucci, B.; Hratchian, H. P.; Ortiz, J. V.; Izmaylov, A. F.; Sonnenberg, J. L.; Williams-Young, D.; Ding, F.; Lipparini, F.; Egidi, F.; Goings, J.; Peng, B.; Petrone, A.; Henderson, T.; Ranasinghe, D.; Zakrzewski, V. G.; Gao, J.; Rega, N.; Zheng, G.; Liang, W.; Hada, M.; Ehara, M.; Toyota, K.; Fukuda, R.; Hasegawa, J.; Ishida, M.; Nakajima, T.; Honda, Y.; Kitao, O.; Nakai, H.; Vreven, T.; Throssell, K.; Montgomery, J. A., Jr.; Peralta, J. E.; Ogliaro, F.; Bearpark, M. J.; Heyd, J. J.; Brothers, E. N.; Kudin, K. N.; Staroverov, V. N.; Keith, T. A.; Kobayashi, R.; Normand, J.; Raghavachari, K.; Rendell, A. P.; Burant, J. C.; Iyengar, S. S.; Tomasi, J.; Cossi, M.; Millam, J. M.; Klene, M.; Adamo, C.; Cammi, R.;

Ochterski, J. W.; Martin, R. L.; Morokuma, K.; Farkas, O.; Foresman, J. B.; Fox, D. J. Gaussian, Inc., Wallingford CT, 2016.

(2) Shao, Y.; Molnar, L. F.; Jung, Y.; Kussmann, J.; Ochsenfeld, C.; Brown, S. T. Phys. Chem. Chem. Phys. 2006, 8, 3172-3191. 
IV) ${ }^{1} \mathrm{H}$ NMR, ${ }^{13} \mathrm{C}$ NMR selected spectra and HPLC traces 

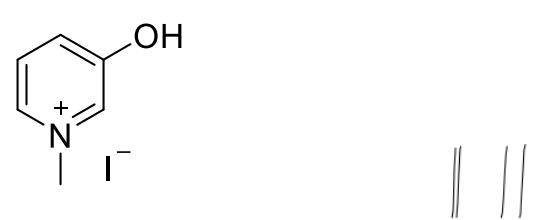

4a, ${ }^{1}$ H NMR

$600 \mathrm{MHz}, \mathrm{DMSO}-d_{6}$
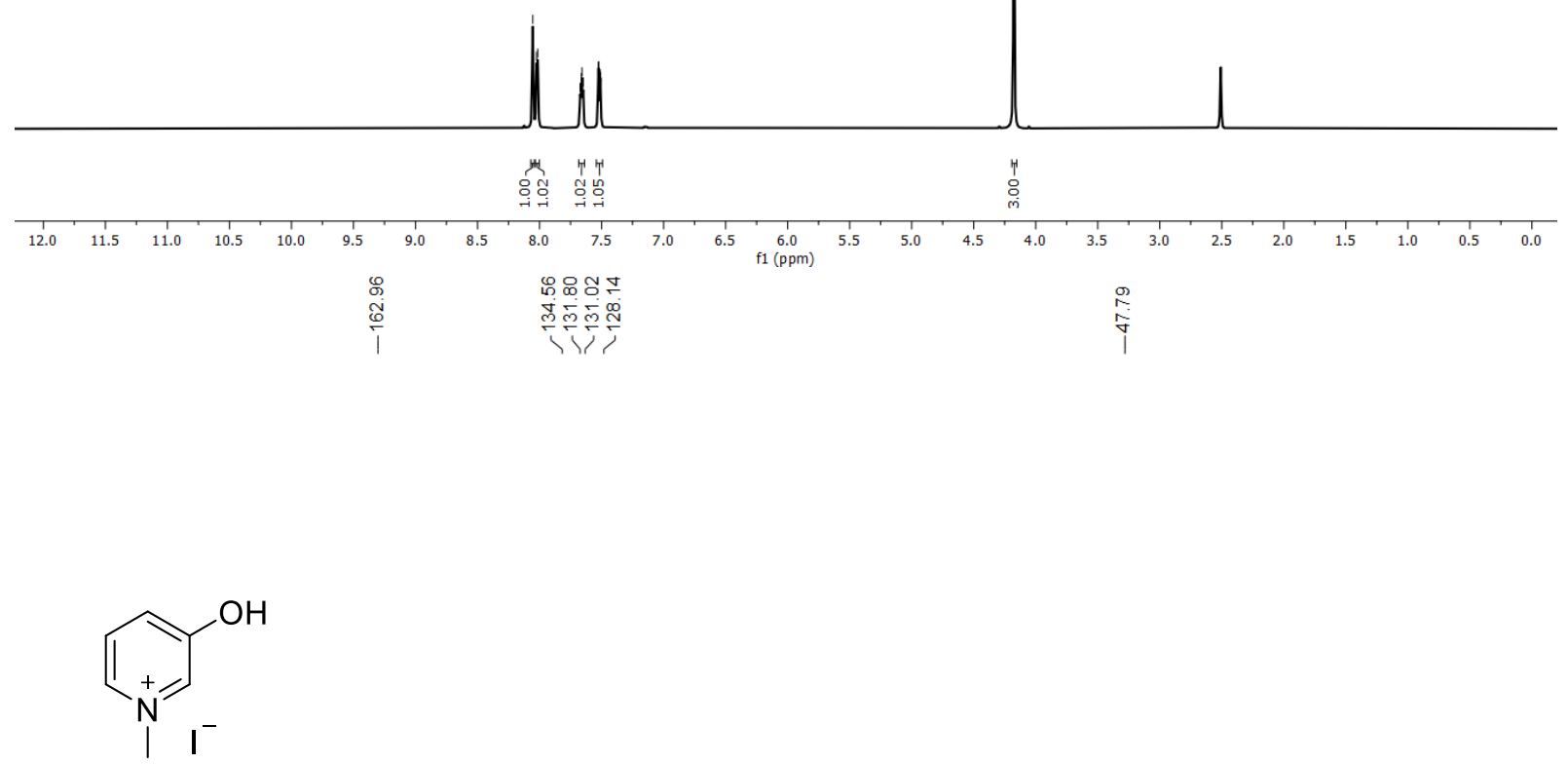

$4 a,{ }^{13} \mathrm{C}\left\{{ }^{1} \mathrm{H}\right\}$ NMR

$151 \mathrm{MHz}, \mathrm{DMSO}-d_{6}$

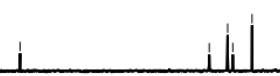

$\begin{array}{llllllllllll}210 & 200 & 190 & 180 & 170 & 160 & 150 & 140 & 130 & 120 & 110 & 100 \\ \mathrm{f} 1(\mathrm{ppm}) & 90\end{array}$ 


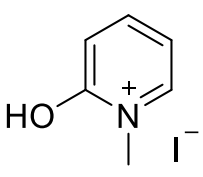

4b, ${ }^{1} \mathrm{H}$ NMR
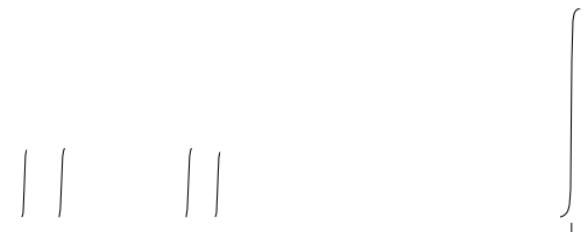

$600 \mathrm{MHz}, \mathrm{DMSO}-d_{6}$
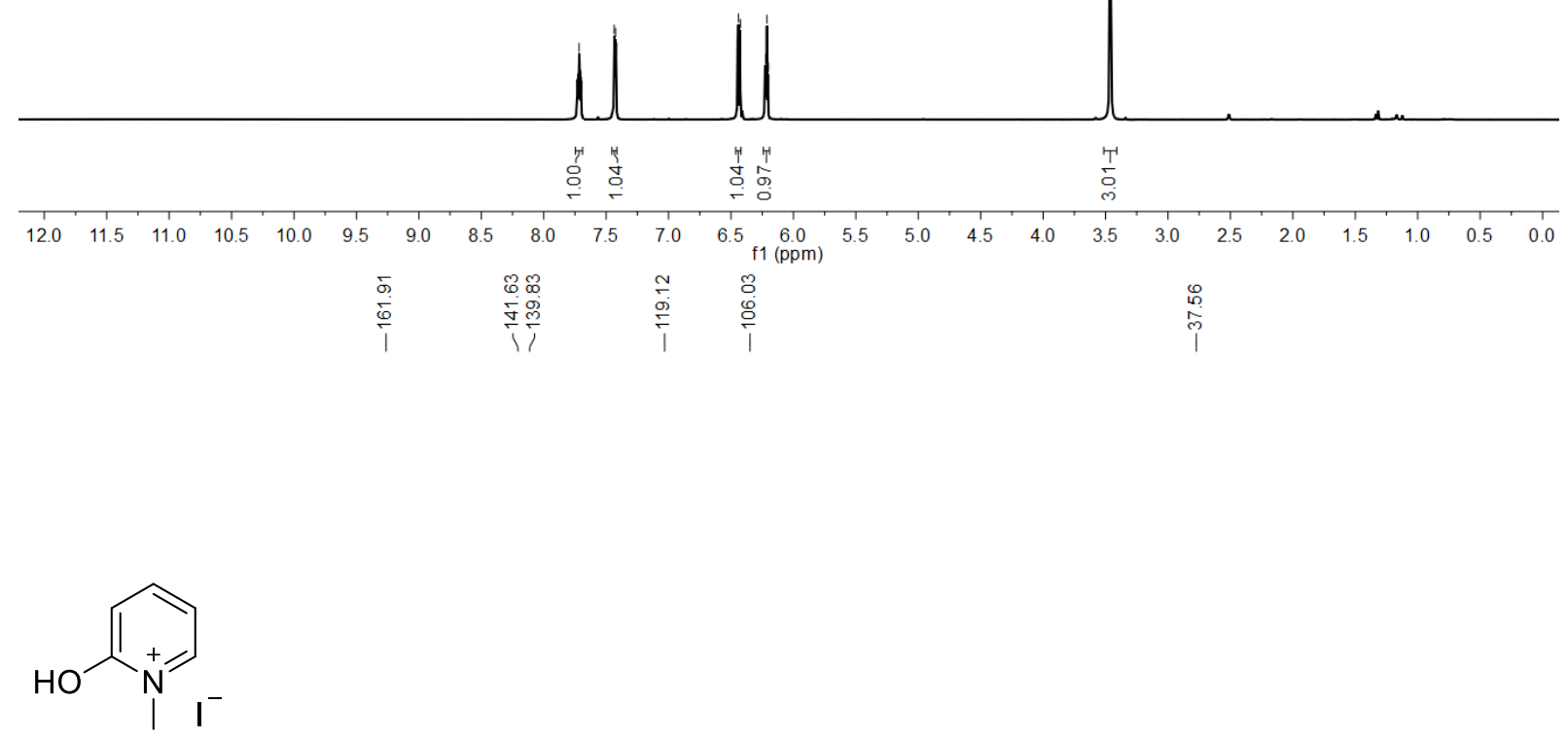

4b, ${ }^{13} \mathrm{C}\left\{{ }^{1} \mathrm{H}\right\}$ NMR

$151 \mathrm{MHz}, \mathrm{DMSO}-d_{6}$

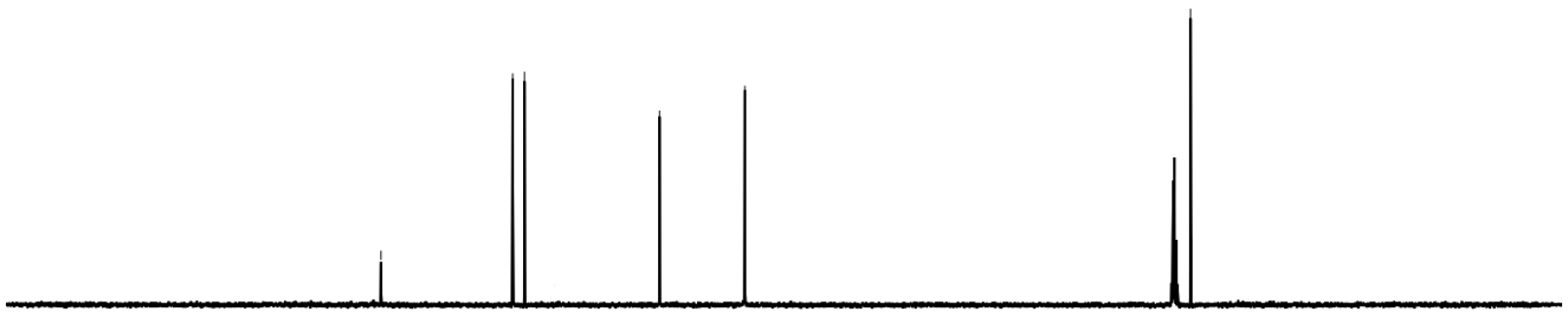

$\begin{array}{lllllllllllllllllllllllll}210 & 200 & 190 & 180 & 170 & 160 & 150 & 140 & 130 & 120 & 110 & 100 & 90 & 80 & 70 & 60 & 50 & 40 & 30 & 20 & 10 & 0 & -10 & \end{array}$ 
<smiles>C[n+]1ccc(O)cc1</smiles>

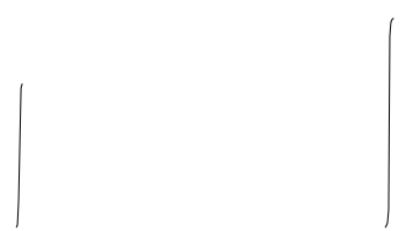

4c, ${ }^{1} \mathrm{H}$ NMR $600 \mathrm{MHz}, \mathrm{DMSO}-d_{6}$

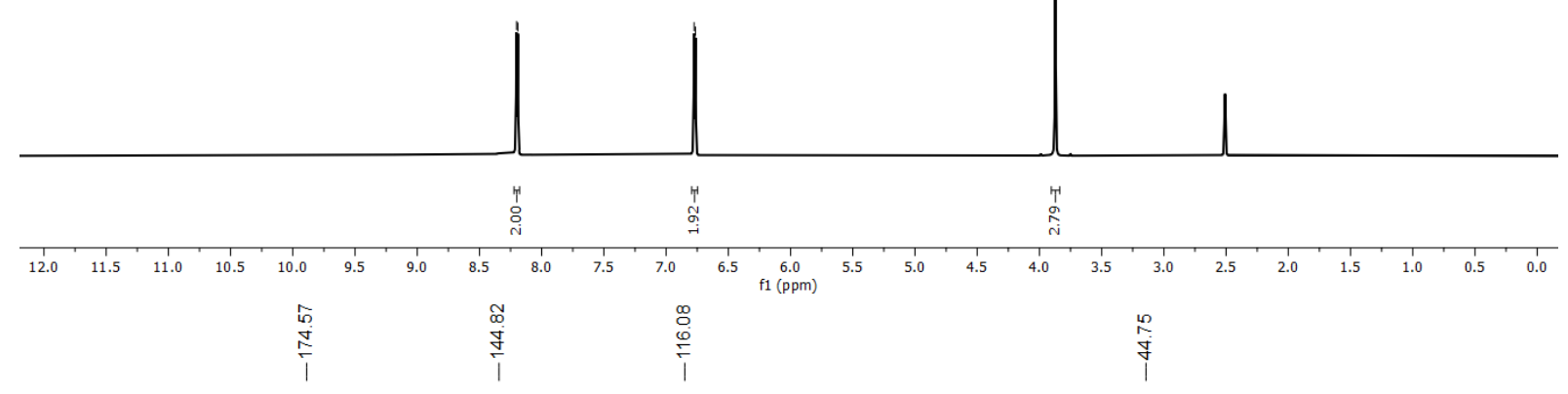<smiles>C[n+]1ccc(O)cc1</smiles>

4c, ${ }^{13} \mathrm{C}\left\{{ }^{1} \mathrm{H}\right\}$ NMR

$600 \mathrm{MHz}, \mathrm{DMSO}-d_{6}$
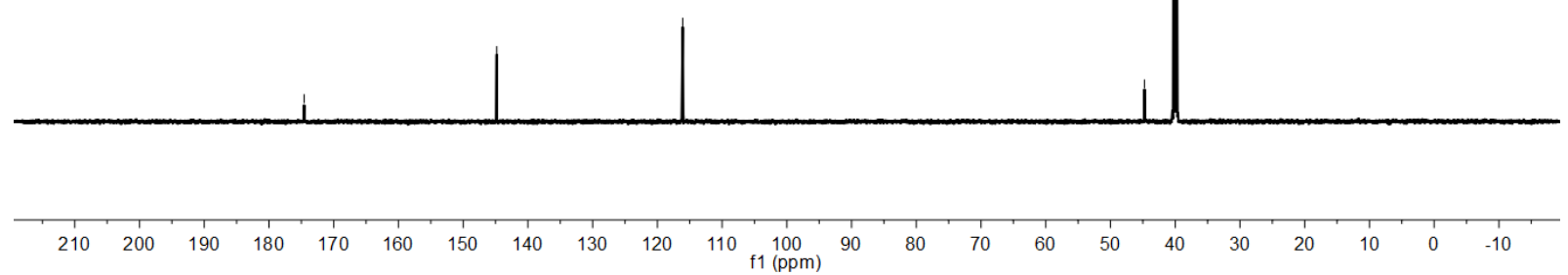


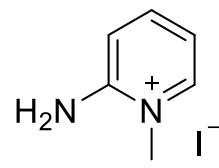

5a, ${ }^{1}$ H NMR
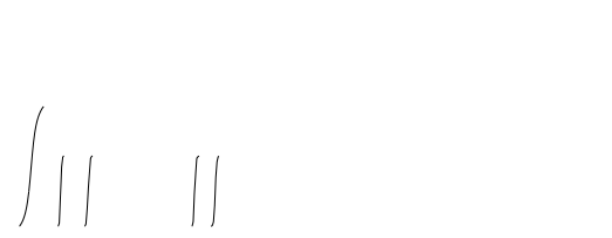

$600 \mathrm{MHz}, \mathrm{DMSO}-d_{6}$
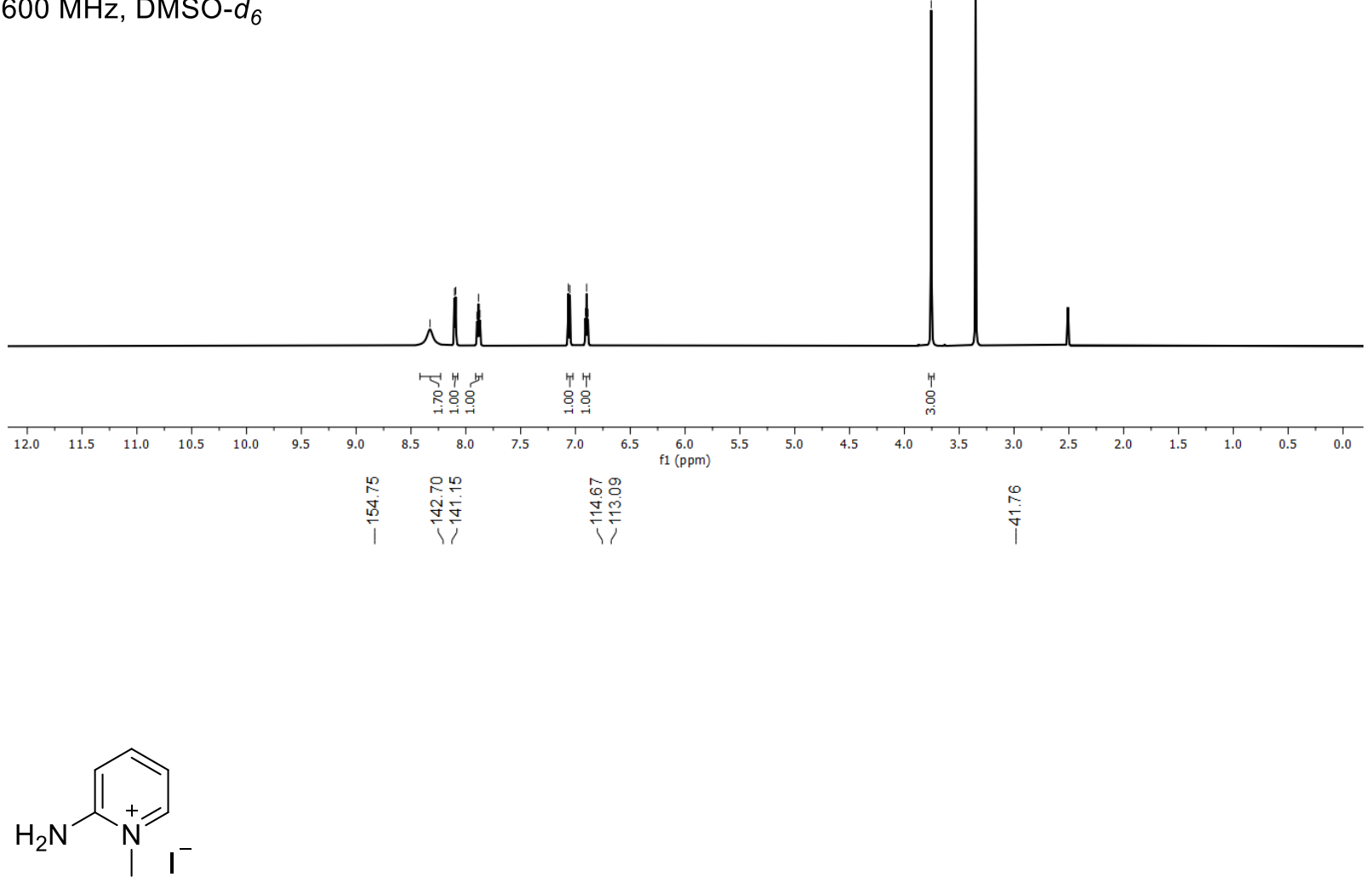

\section{5a, ${ }^{13} \mathrm{C}\left\{{ }^{1} \mathrm{H}\right\}$ NMR}

$151 \mathrm{MHz}, \mathrm{DMSO}-d_{6}$
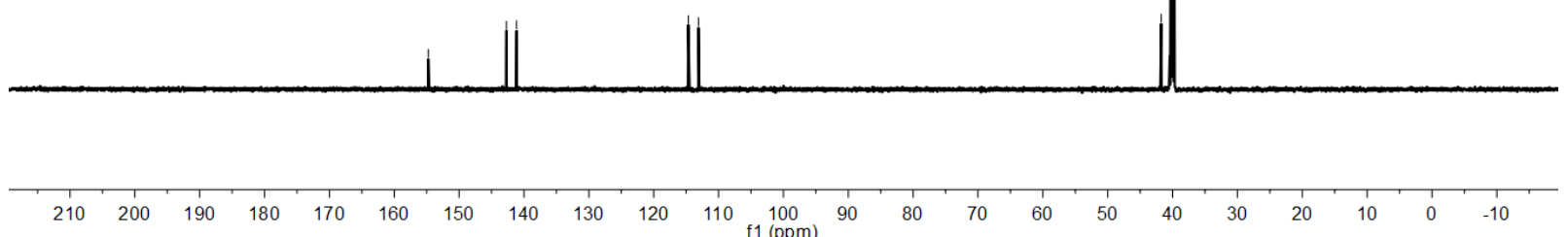


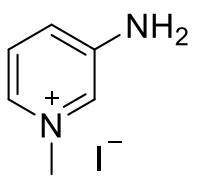

5b, ${ }^{1} \mathrm{H}$ NMR

$600 \mathrm{MHz}, \mathrm{DMSO}-d_{6}$
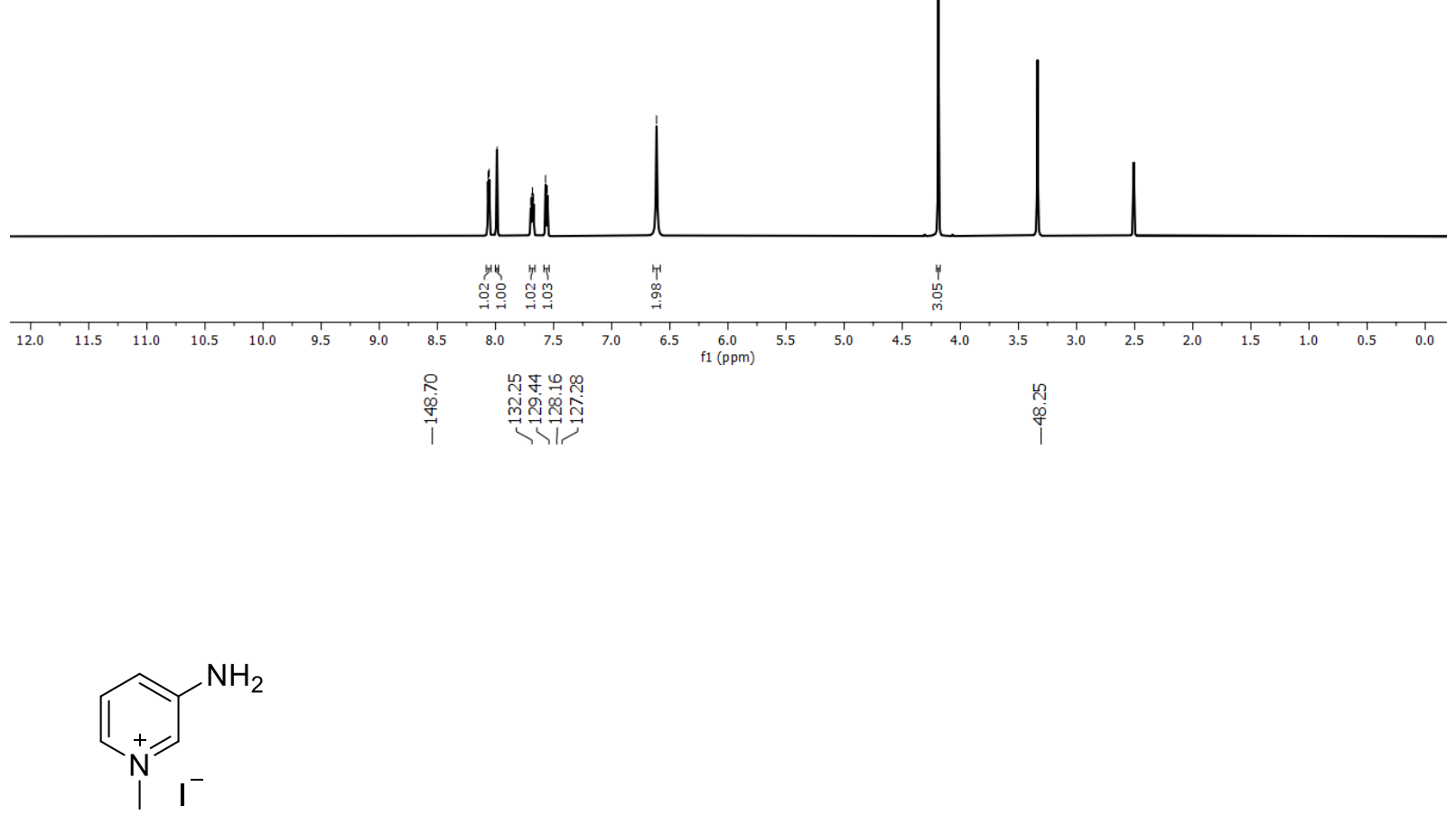

$5 b,{ }^{13} \mathrm{C}\left\{{ }^{1} \mathrm{H}\right\}$ NMR

$151 \mathrm{MHz}, \mathrm{DMSO}^{-d_{6}}$ 


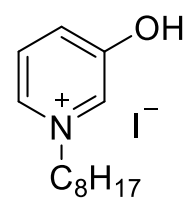

6a, ${ }^{1} \mathrm{H}$ NMR

$600 \mathrm{MHz}, \mathrm{CDCl}_{3}$

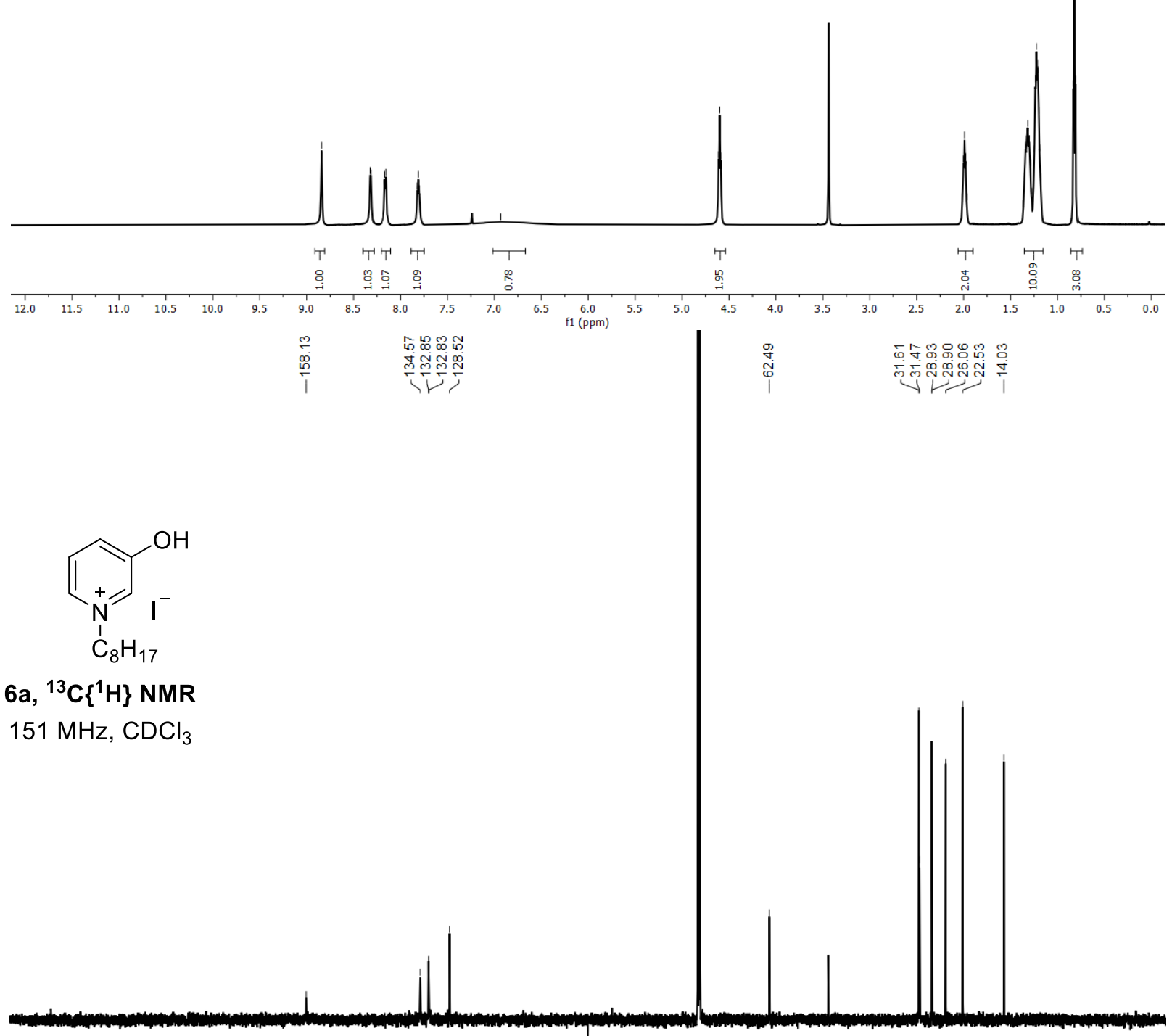

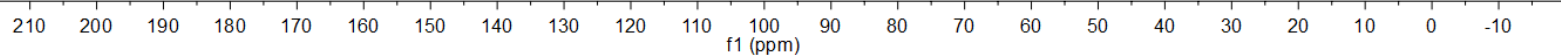



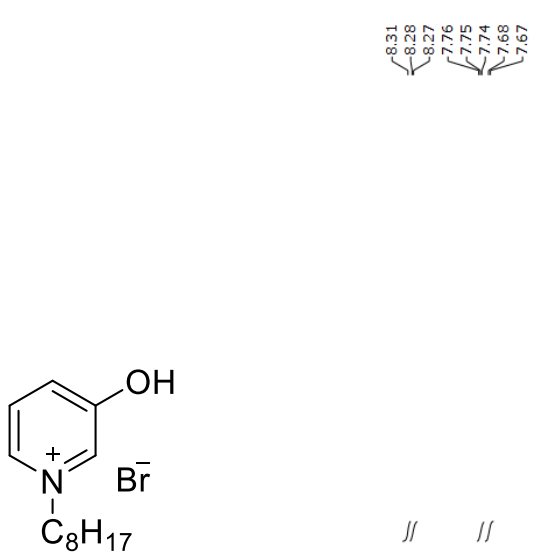

6b, ${ }^{1} \mathrm{H}$ NMR

$600 \mathrm{MHz}, \mathrm{DMSO}-d_{6}$
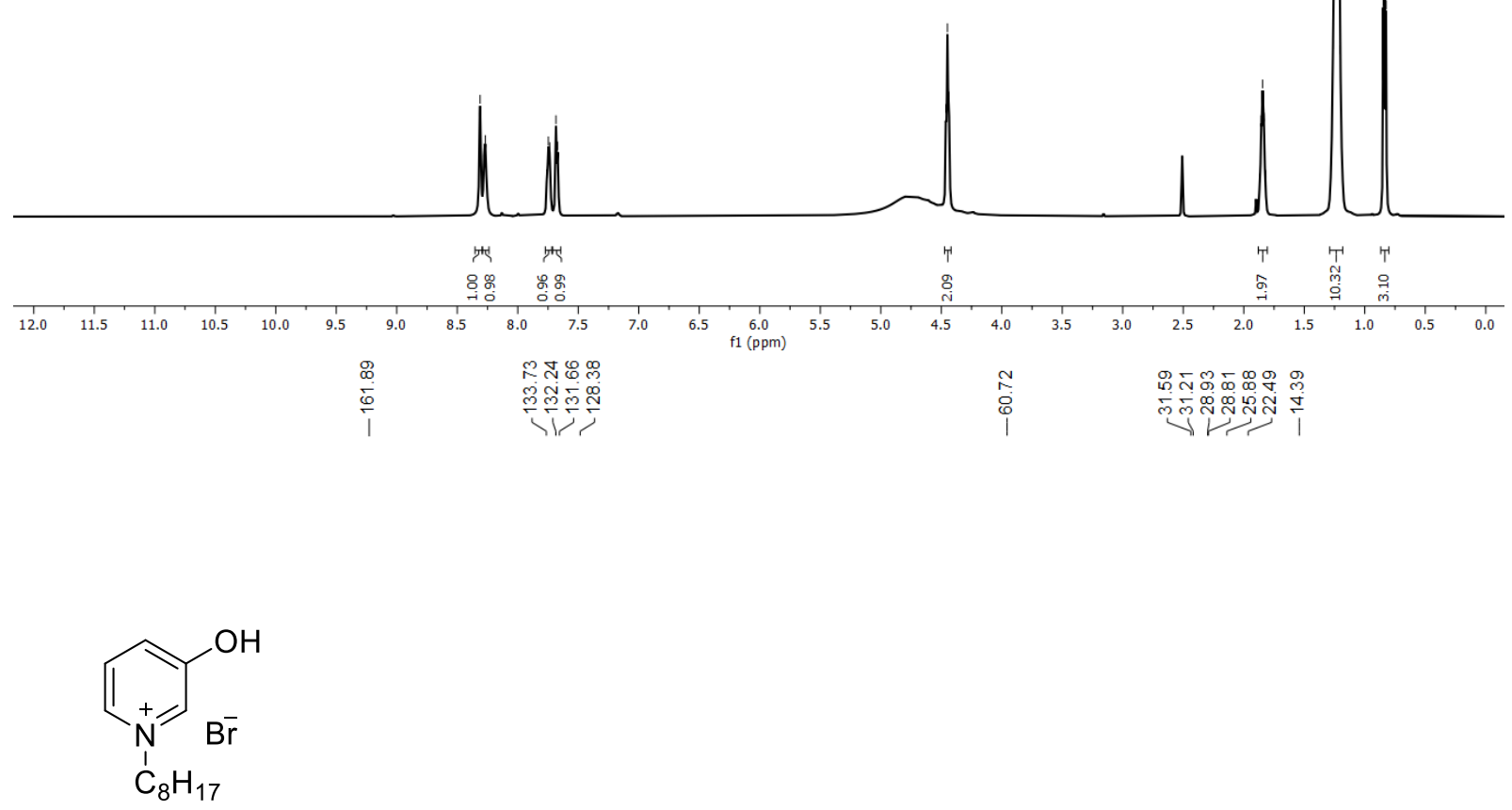

6b, ${ }^{13} \mathrm{C}\left\{{ }^{1} \mathrm{H}\right\}$ NMR

$151 \mathrm{MHz}, \mathrm{DMSO}-d_{6}$

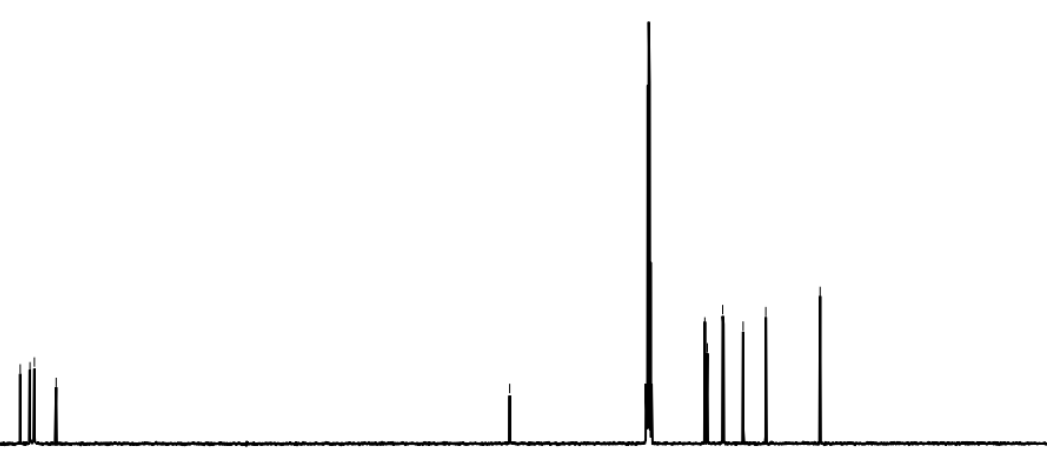

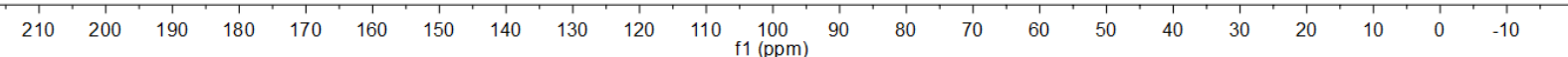




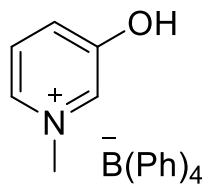

6c, ${ }^{1} \mathrm{H}$ NMR

$600 \mathrm{MHz}, \mathrm{DMSO}-d_{6}$
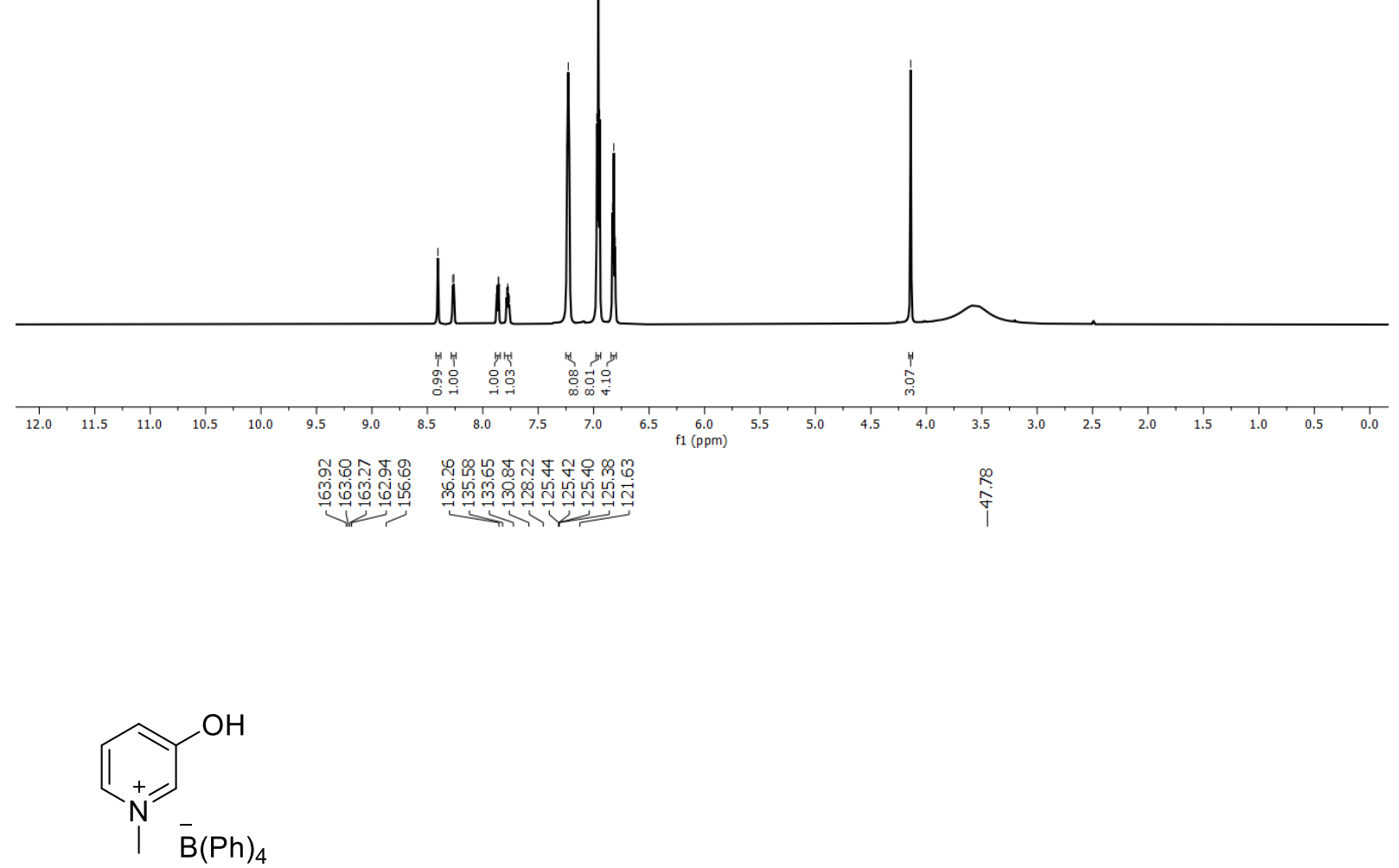

6c, ${ }^{13} \mathrm{C}\left\{{ }^{1} \mathrm{H}\right\}$ NMR

$151 \mathrm{MHz}, \mathrm{DMSO}^{-d_{6}}$

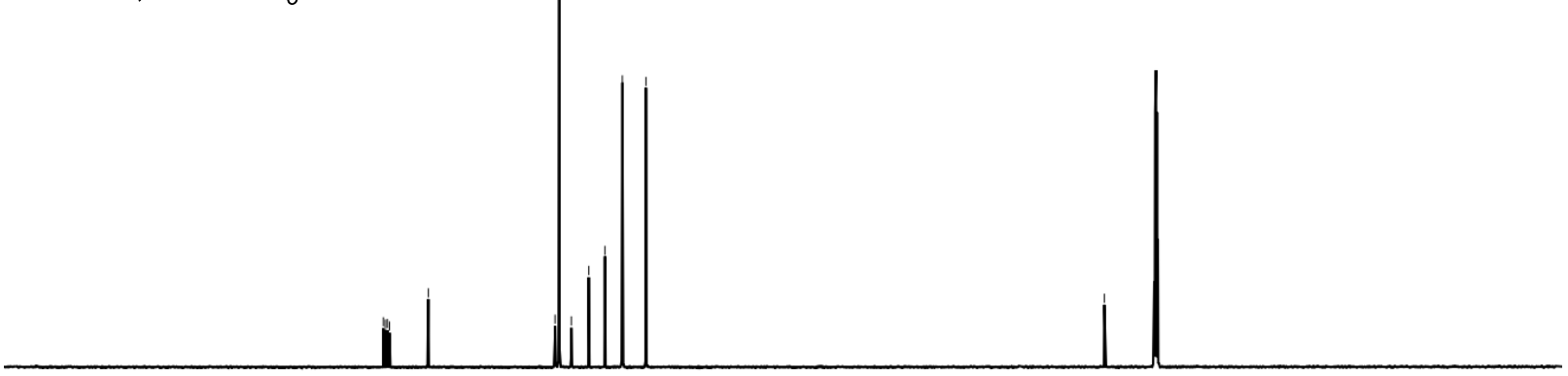

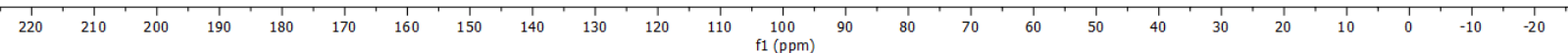




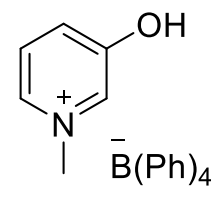

6c, ${ }^{11}$ B NMR

$193 \mathrm{MHz}, \mathrm{DMSO}-d_{6}$

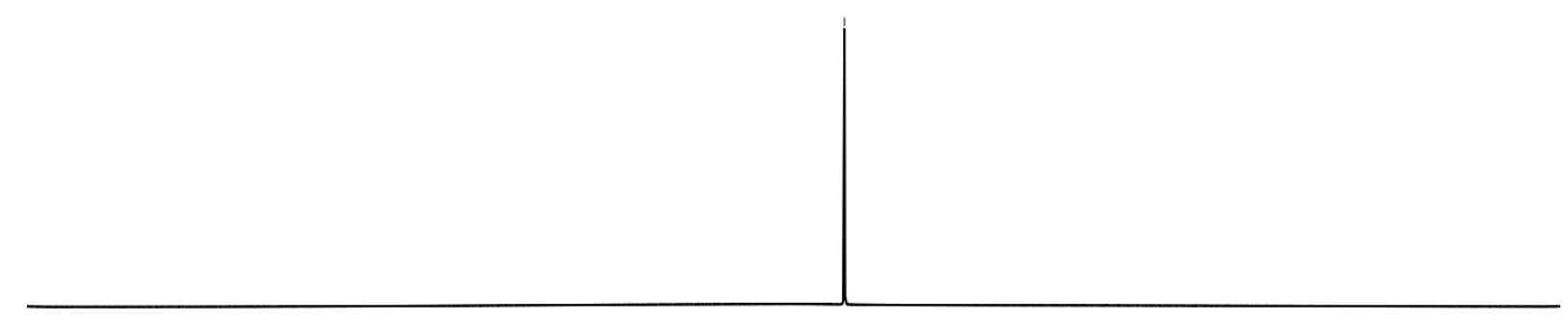

\begin{tabular}{|c|c|c|c|c|c|c|c|c|c|c|c|c|c|c|c|c|c|c|}
\hline 90 & 80 & 70 & 60 & 50 & 40 & 30 & 20 & 10 & $\begin{array}{c}0 \\
\mathrm{f} 1(\mathrm{ppm})\end{array}$ & -10 & -20 & -30 & -40 & -50 & -60 & -70 & -80 & -90 \\
\hline & & & & $\begin{array}{l}8.8 \\
\sigma i \infty \\
\underbrace{\circ}\end{array}$ & 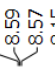 & & & & & & & ஜ্ & & & & & & \\
\hline
\end{tabular}

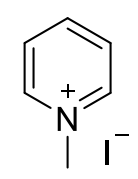

7a, ${ }^{1} \mathrm{H}$ NMR

$600 \mathrm{MHz}, \mathrm{DMSO}-d_{6}$
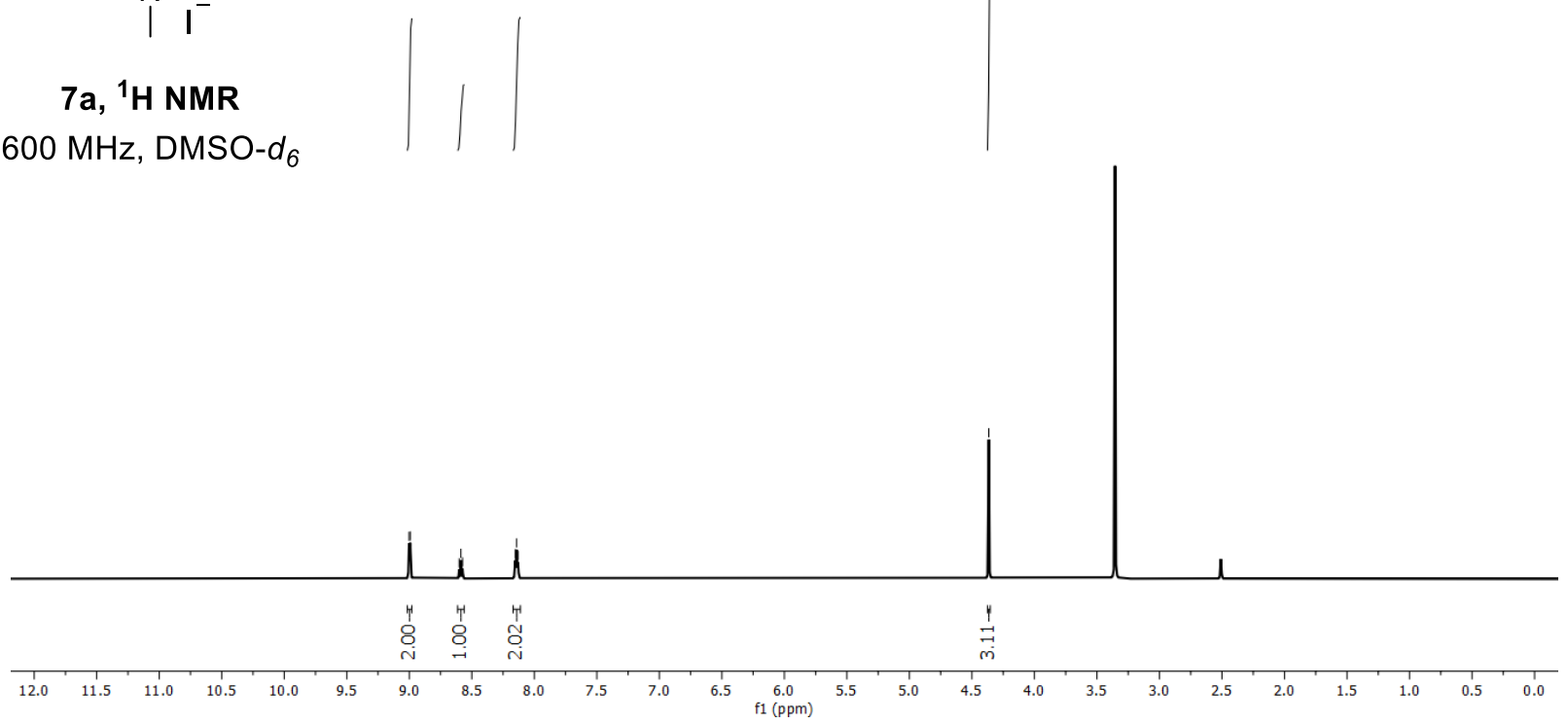


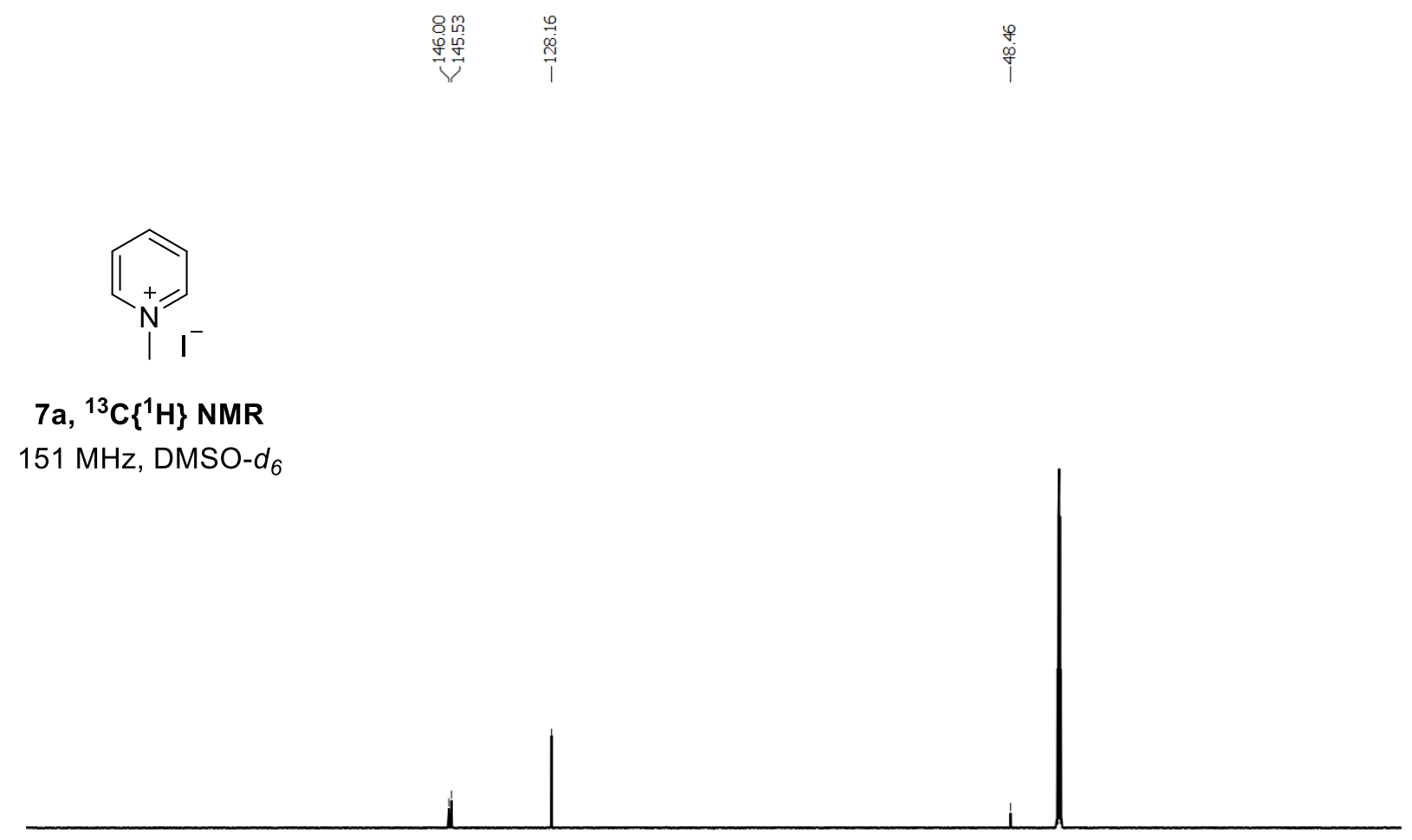

$7 \mathrm{a},{ }^{13} \mathrm{C}\left\{{ }^{1} \mathrm{H}\right\}$ NMR

$151 \mathrm{MHz}, \mathrm{DMSO}-\mathrm{d}_{6}$

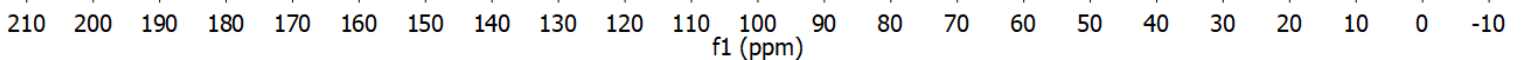

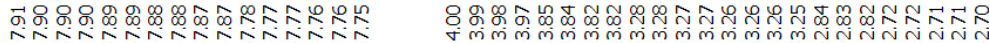

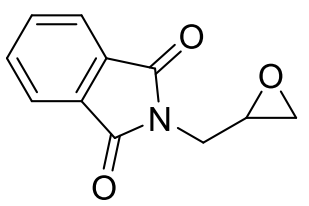

1f, ${ }^{1} \mathrm{H}$ NMR

$600 \mathrm{MHz}, \mathrm{CDCl}_{3}$
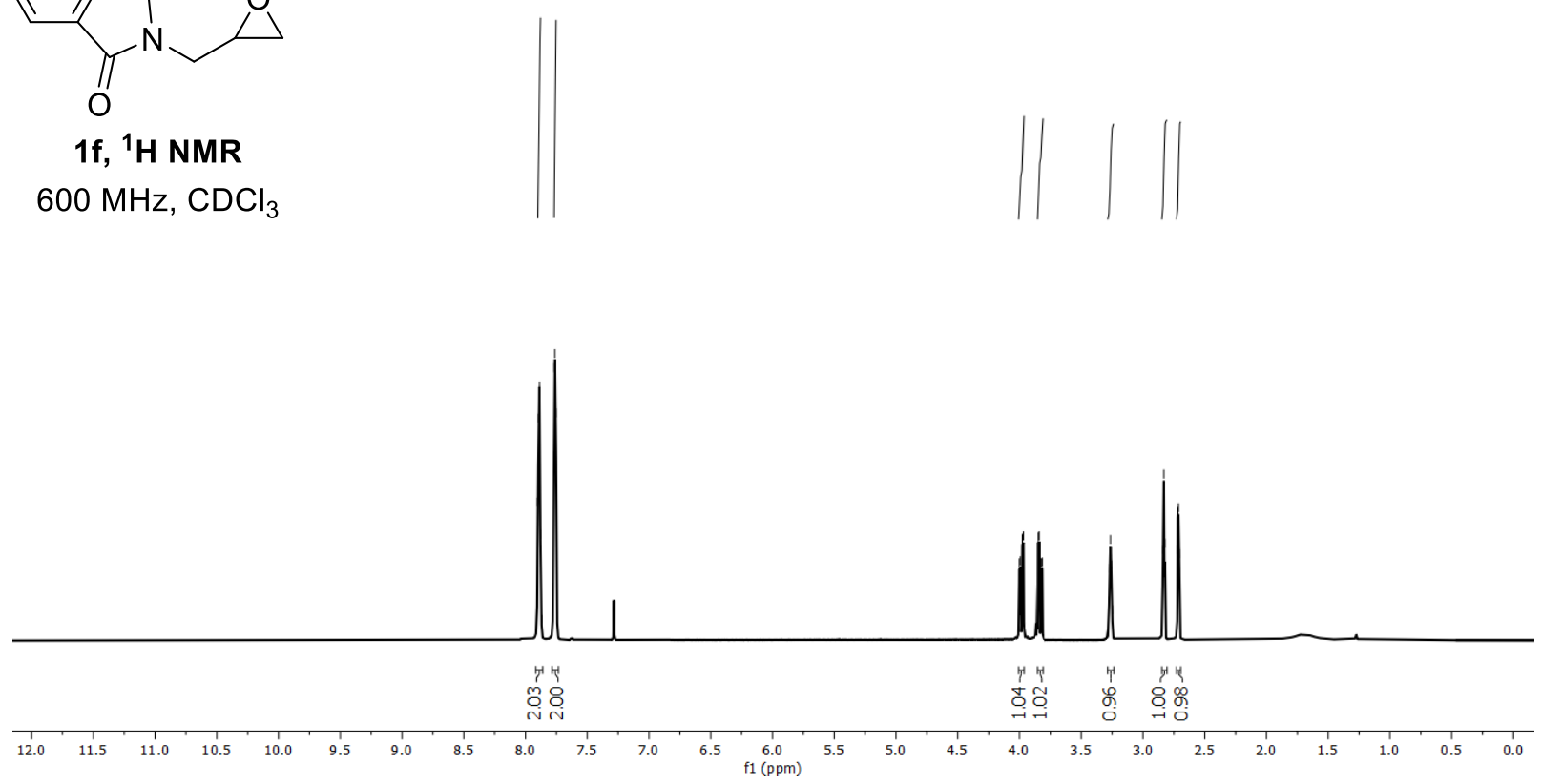

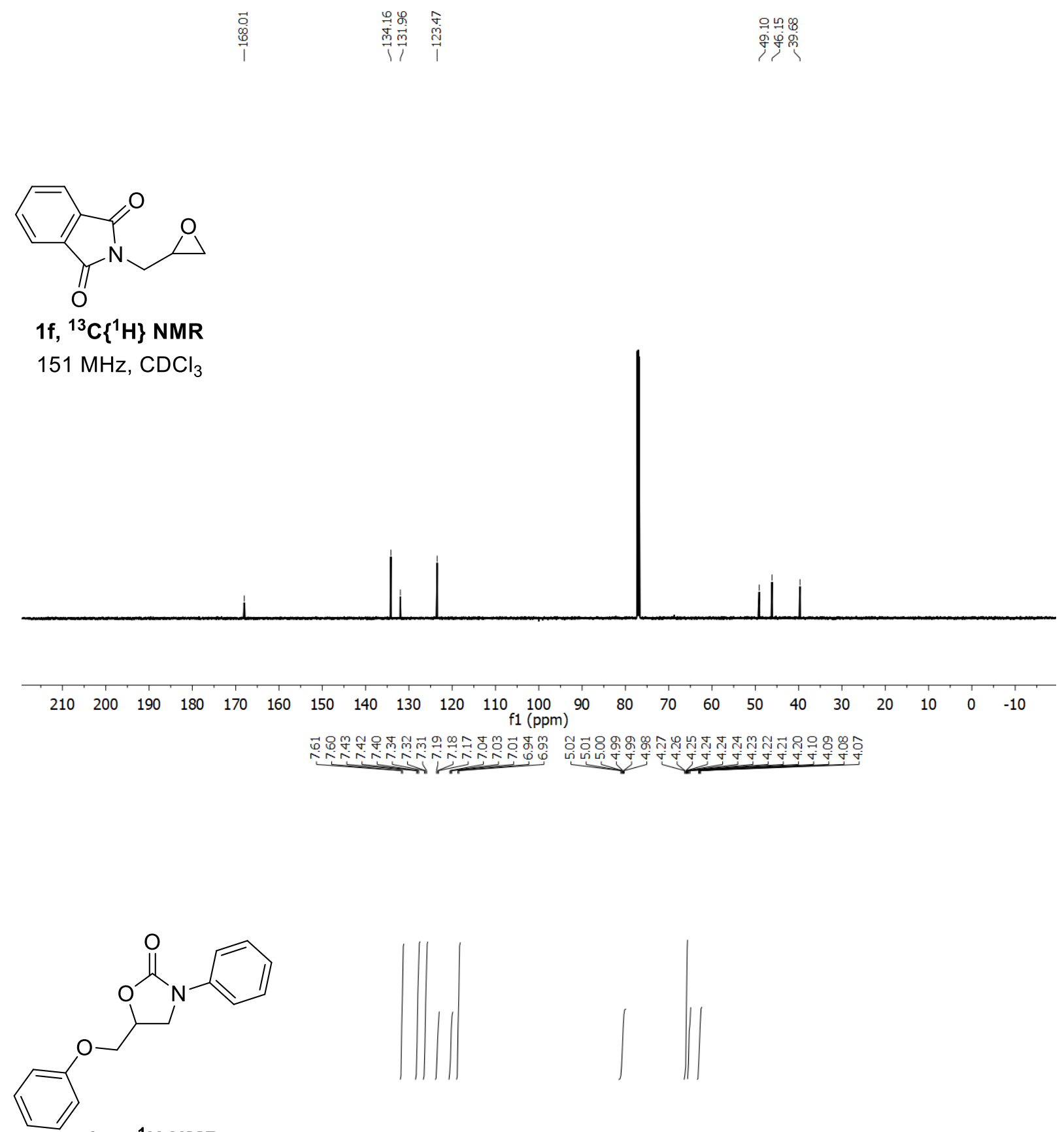

3aa, ${ }^{1} \mathrm{H}$ NMR

$600 \mathrm{MHz}, \mathrm{CDCl}_{3}$

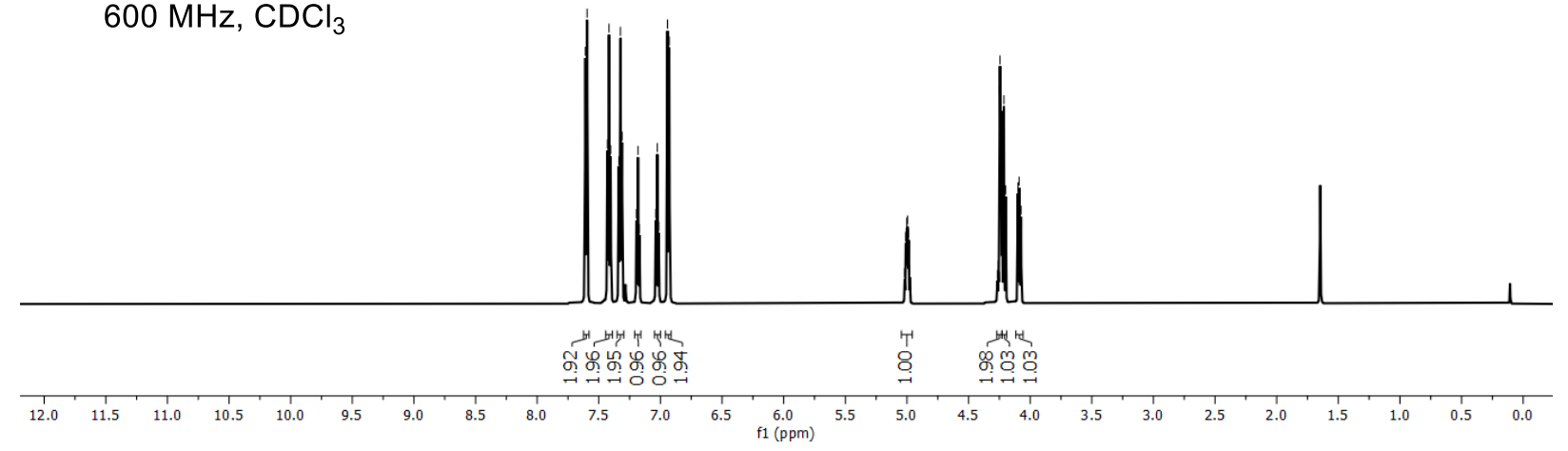



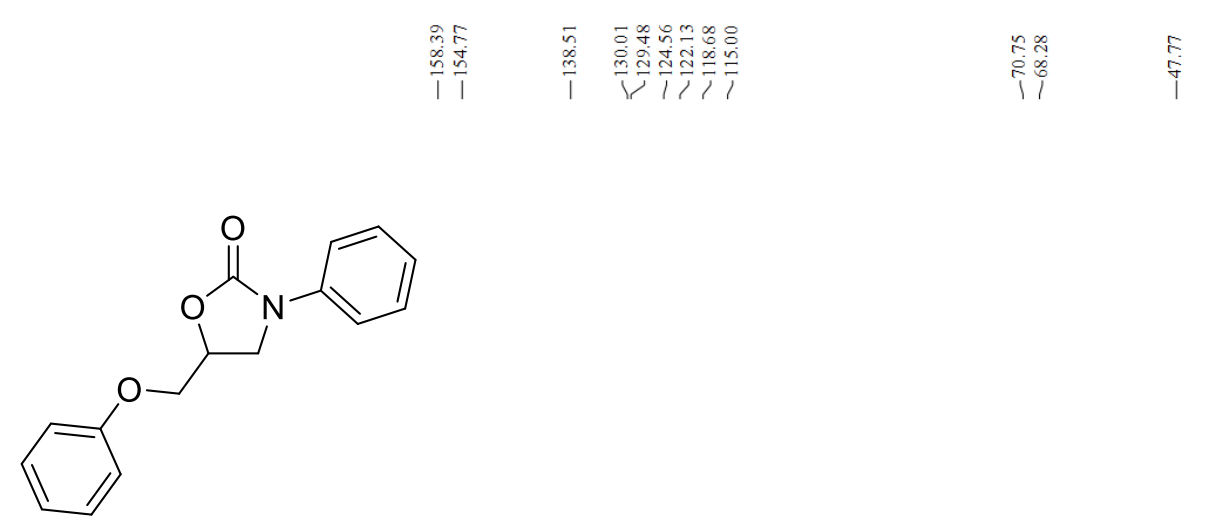

3aa, ${ }^{13} \mathrm{C}\left\{{ }^{1} \mathrm{H}\right\}$ NMR

$151 \mathrm{MHz}, \mathrm{CDCl}_{3}$
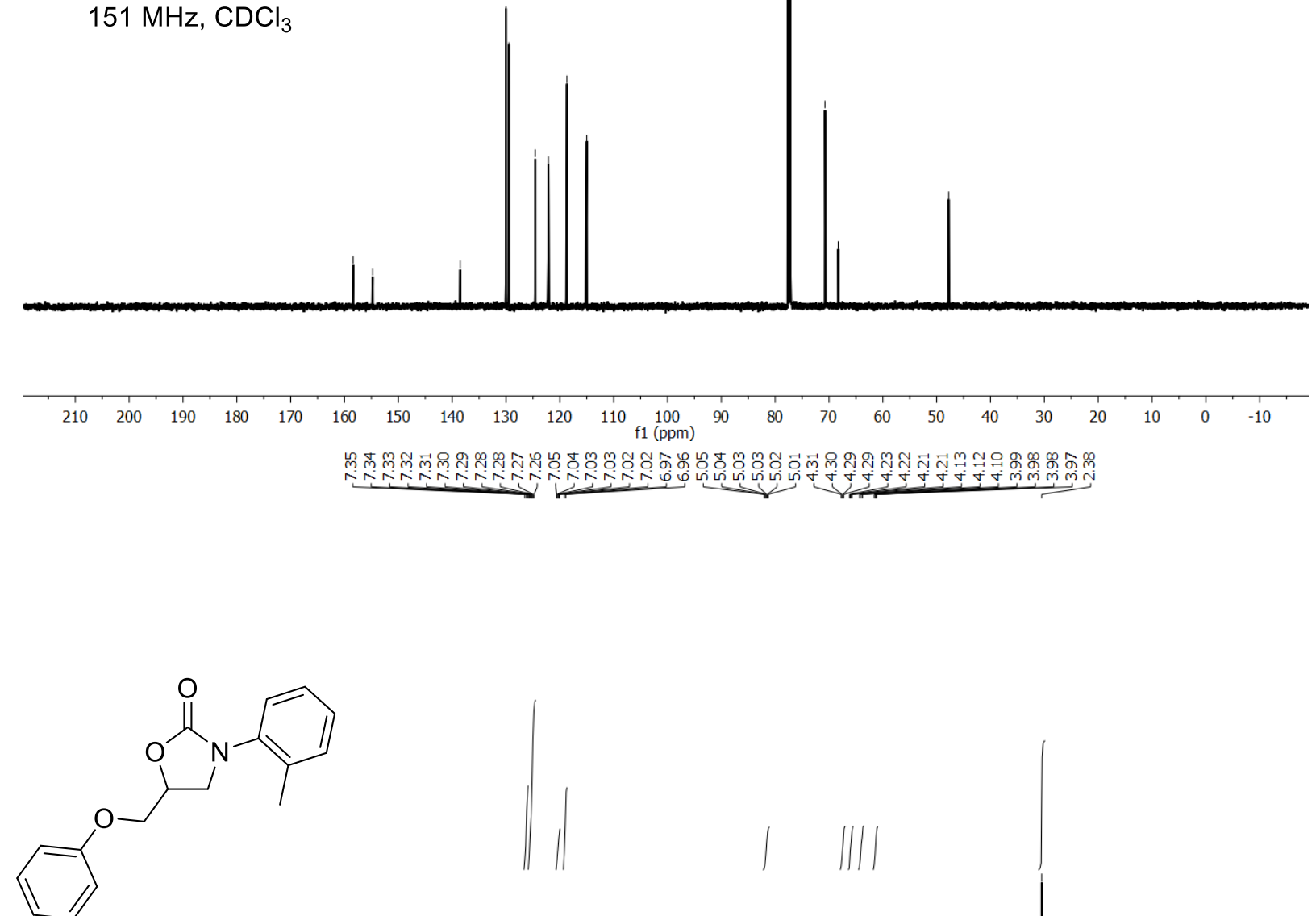

3ab, ${ }^{1} \mathbf{H}$ NMR

$600 \mathrm{MHz}, \mathrm{CDCl}_{3}$

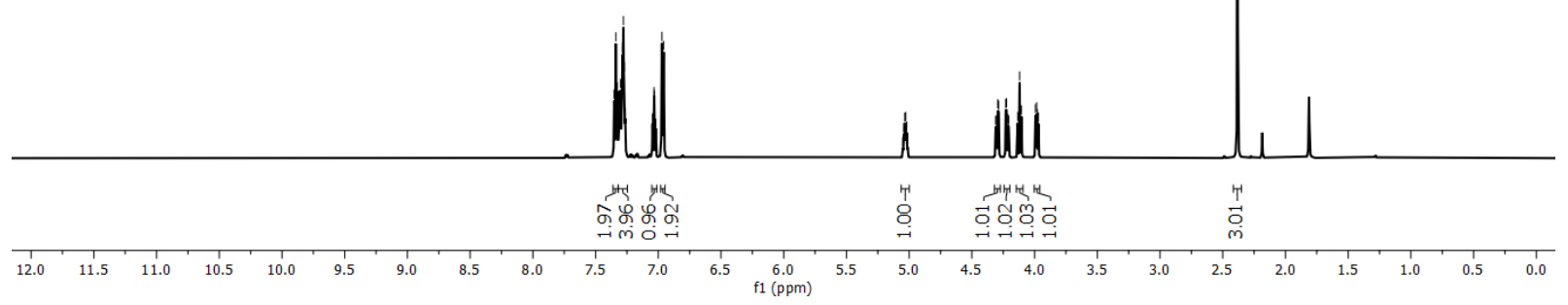




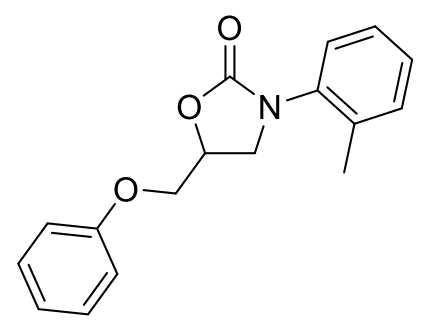

$3 a b,{ }^{13} \mathrm{C}\left\{{ }^{1} \mathrm{H}\right\}$ NMR

$151 \mathrm{MHz}, \mathrm{CDCl}_{3}$
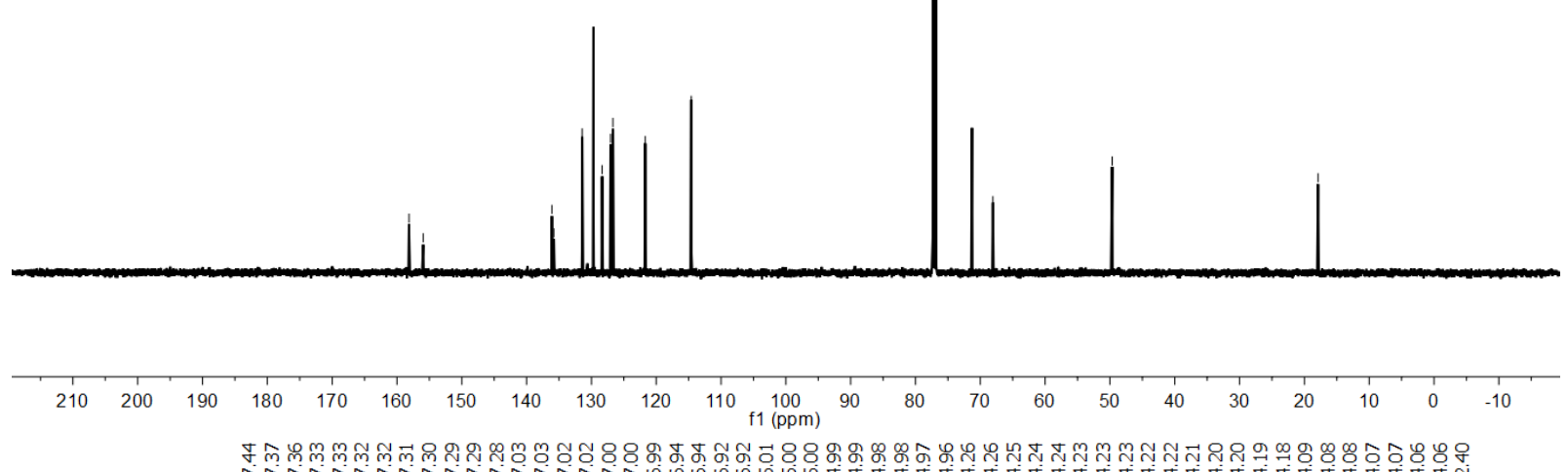

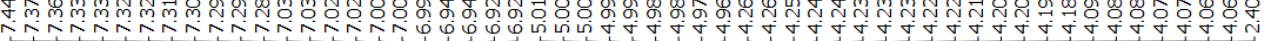
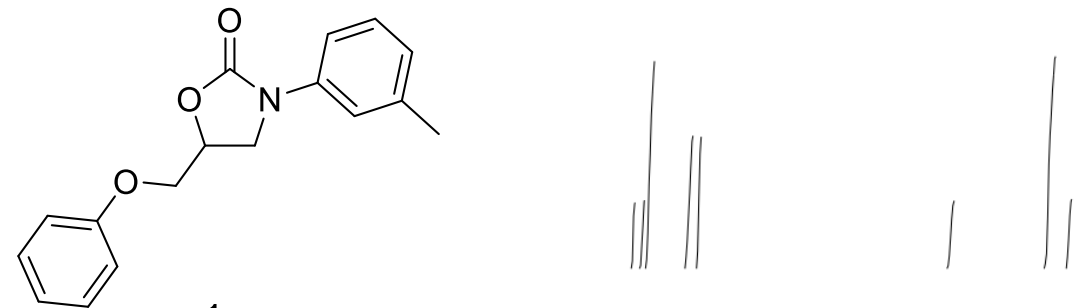

3ac, ${ }^{1} \mathrm{H}$ NMR

$600 \mathrm{MHz}, \mathrm{CDCl}_{3}$

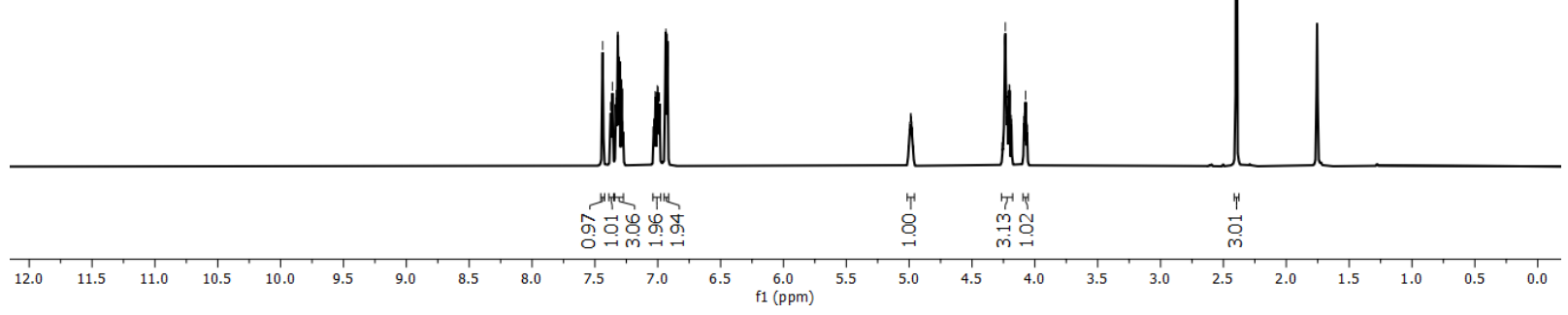



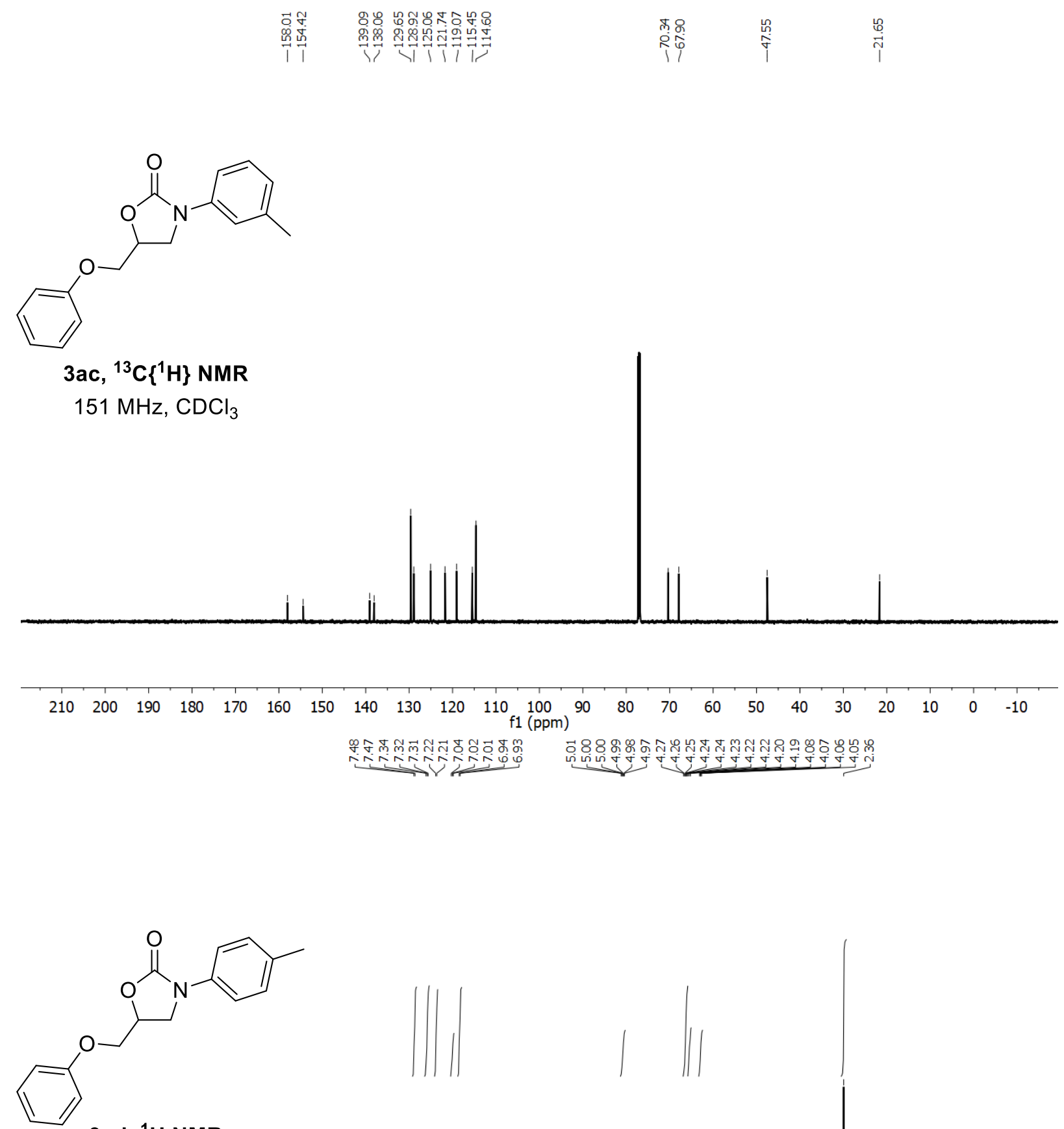

3ad, ${ }^{1} \mathrm{H}$ NMR

$600 \mathrm{MHz}, \mathrm{CDCl}_{3}$

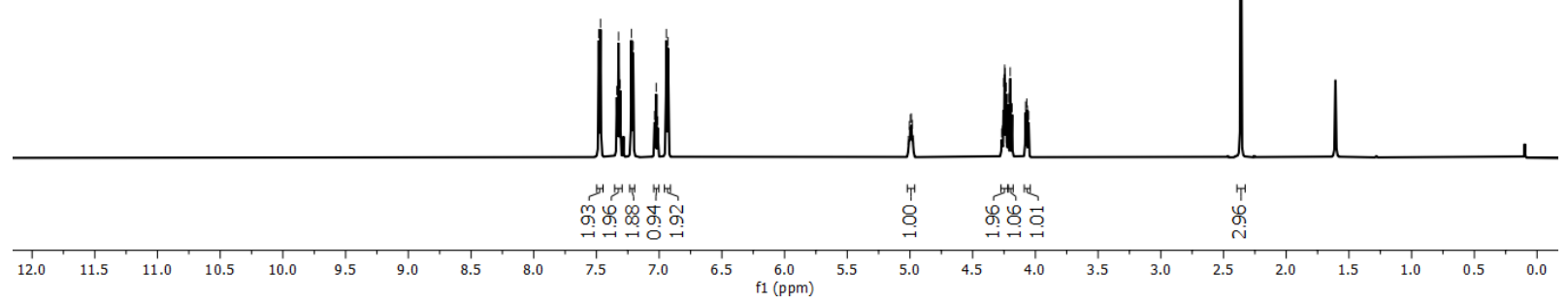



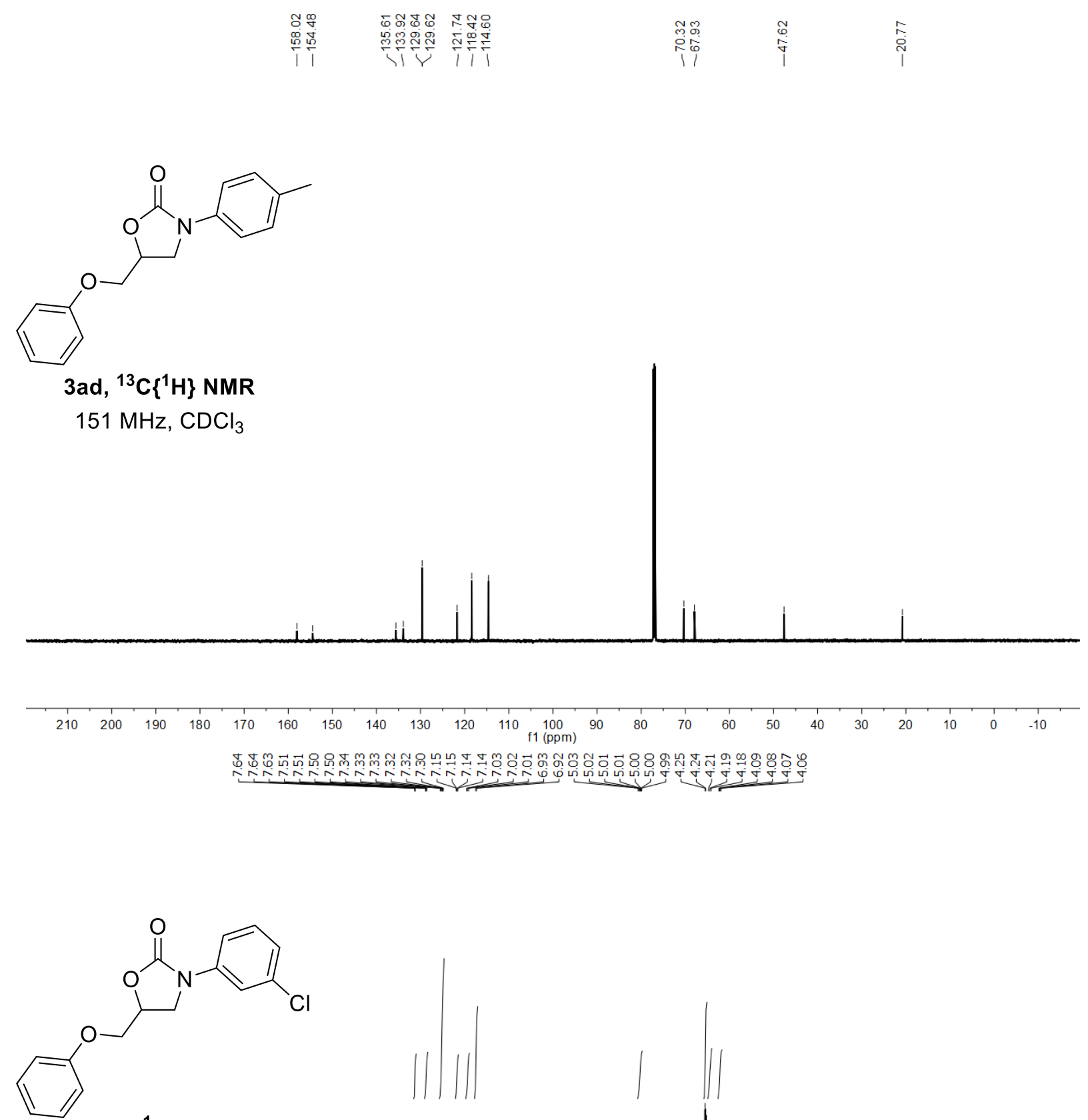

3ae, ${ }^{\mathbf{1}} \mathrm{H}$ NMR

$600 \mathrm{MHz}, \mathrm{CDCl}_{3}$

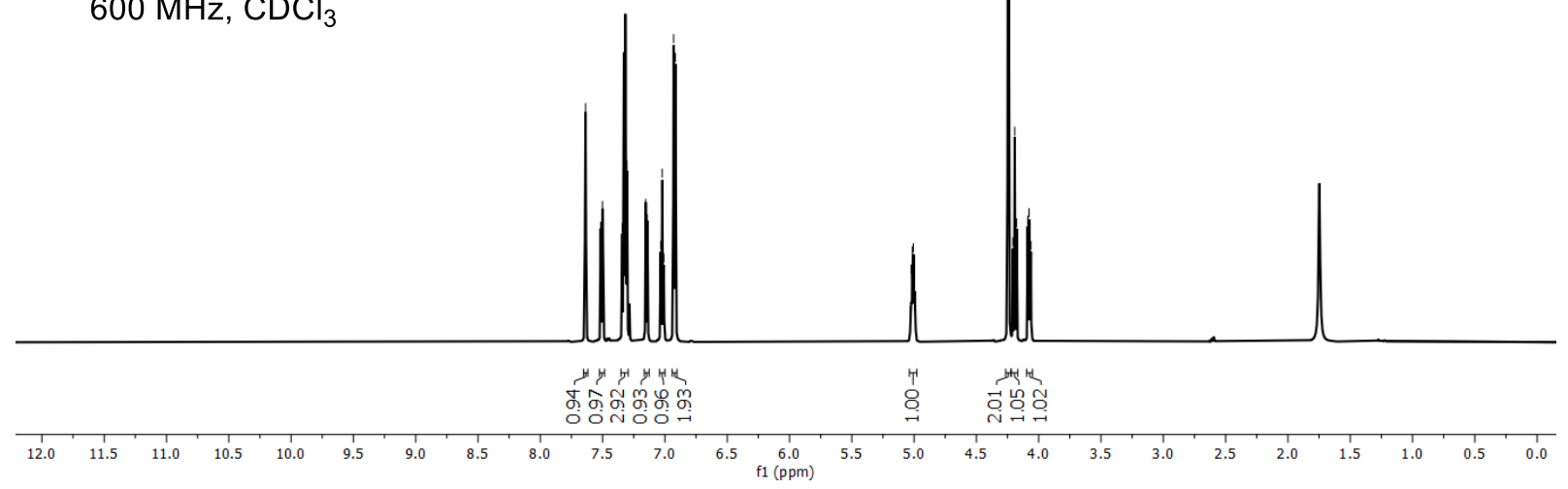



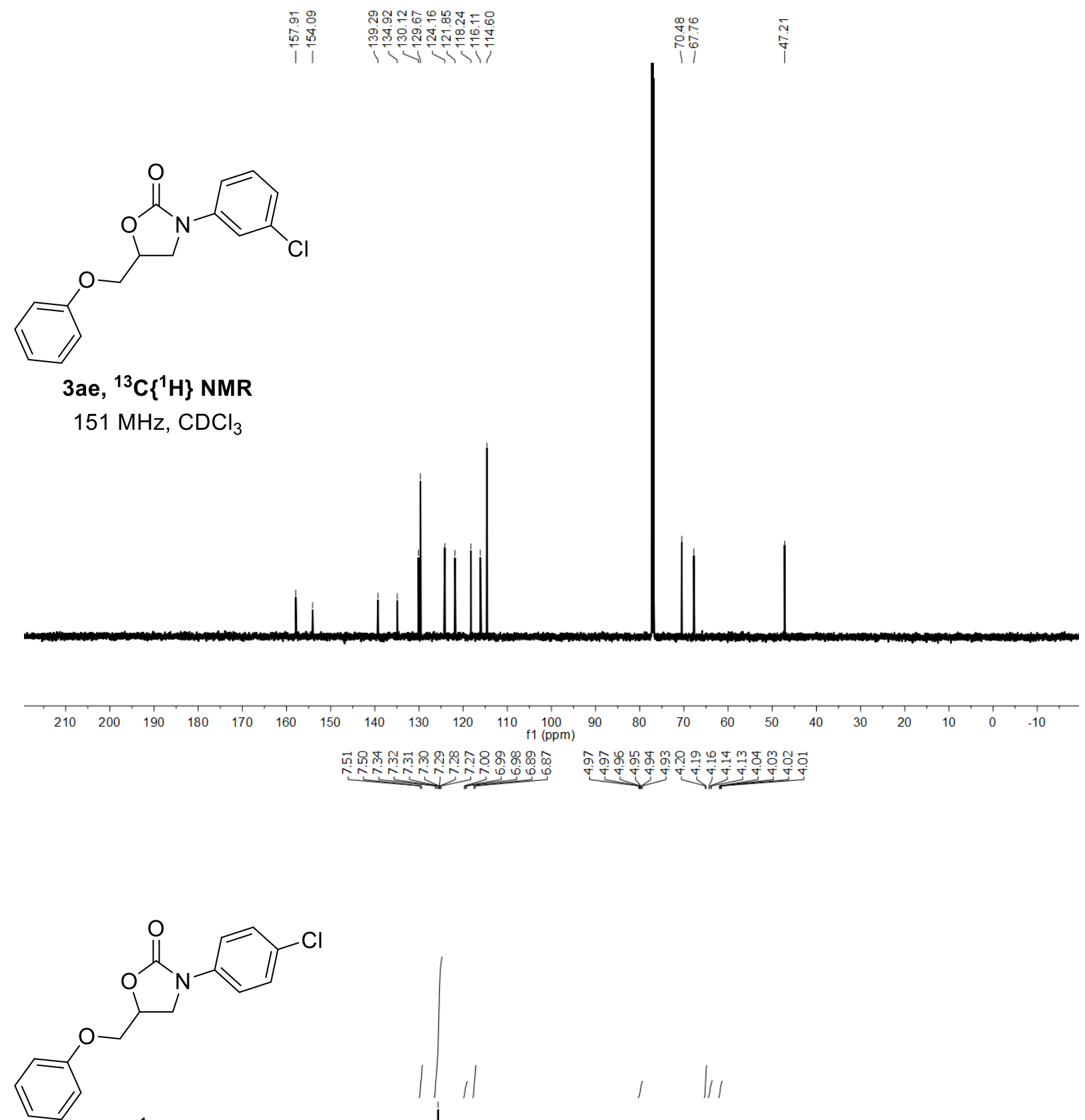

3af, ${ }^{\mathbf{1}} \mathrm{H}$ NMR

$600 \mathrm{MHz}, \mathrm{CDCl}_{3}$

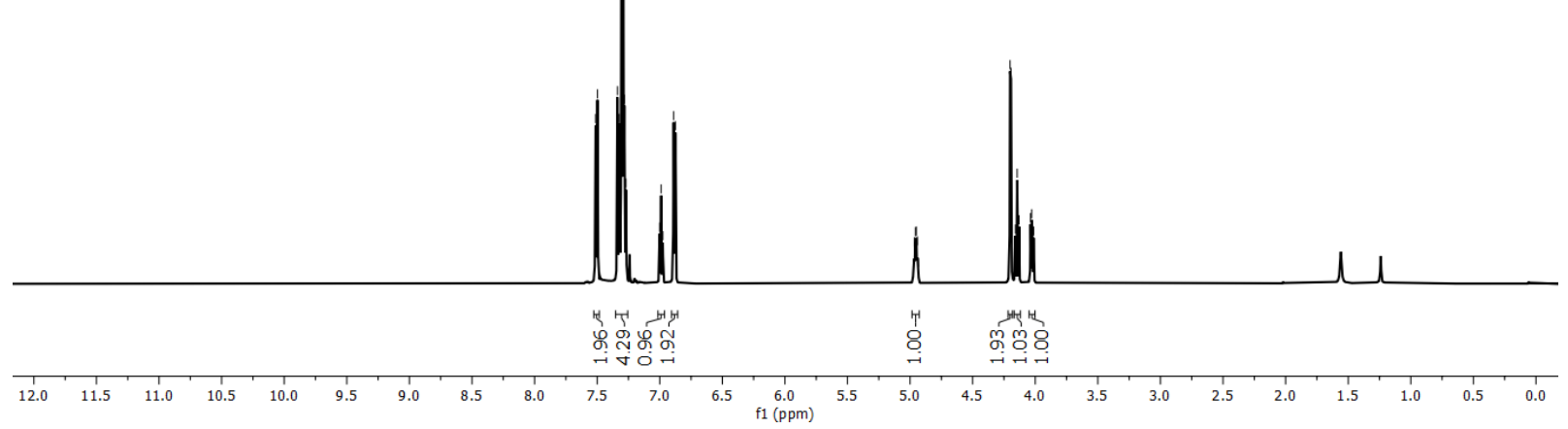



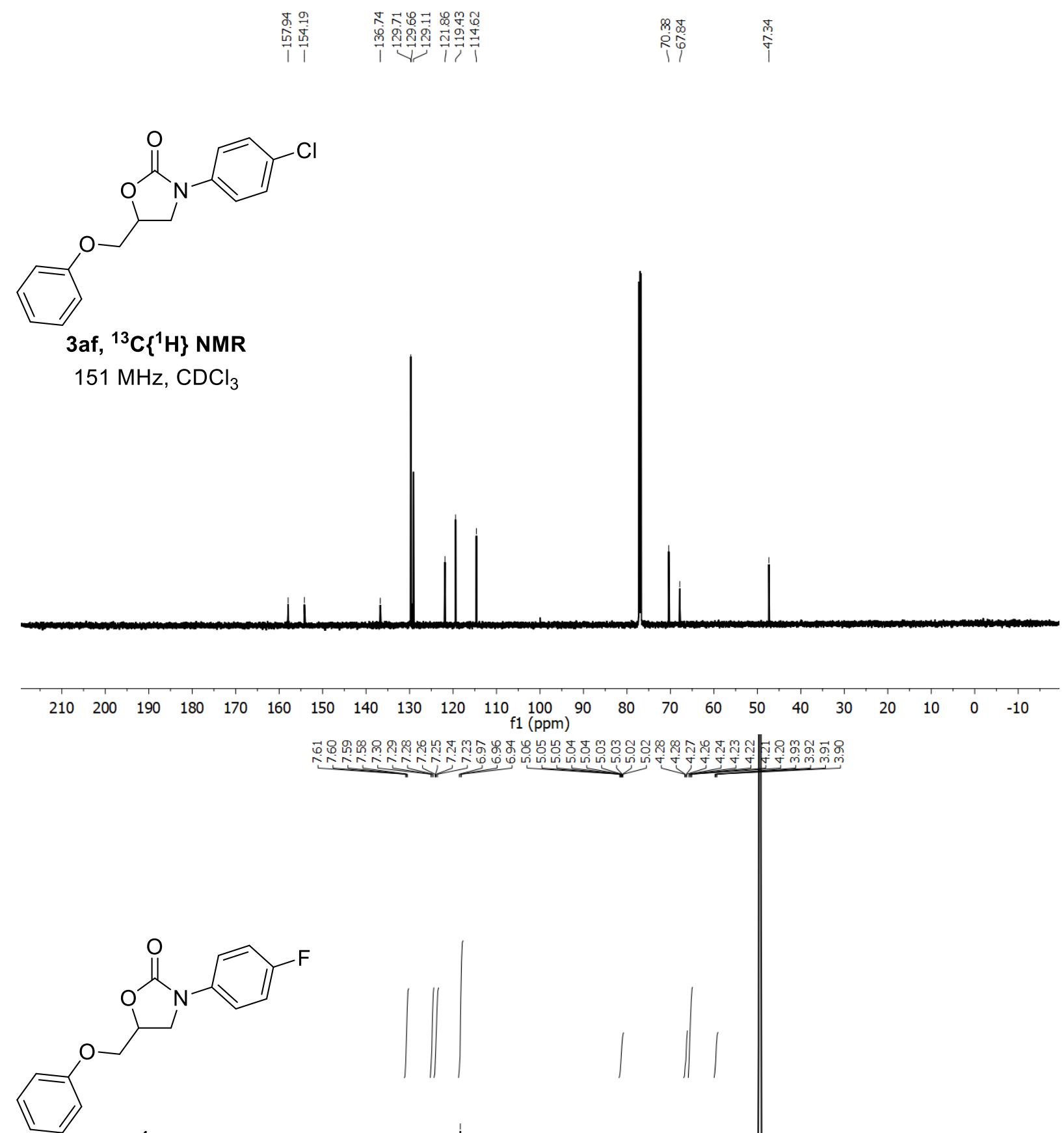

3ag, ${ }^{1} \mathrm{H}$ NMR

$600 \mathrm{MHz}, \mathrm{DMSO}-d_{6}$

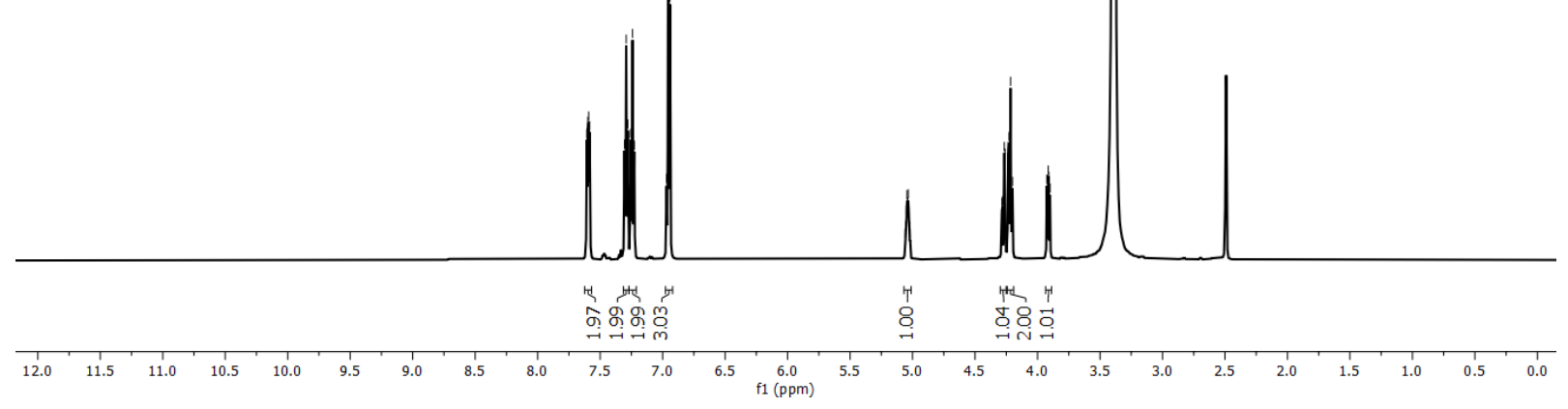



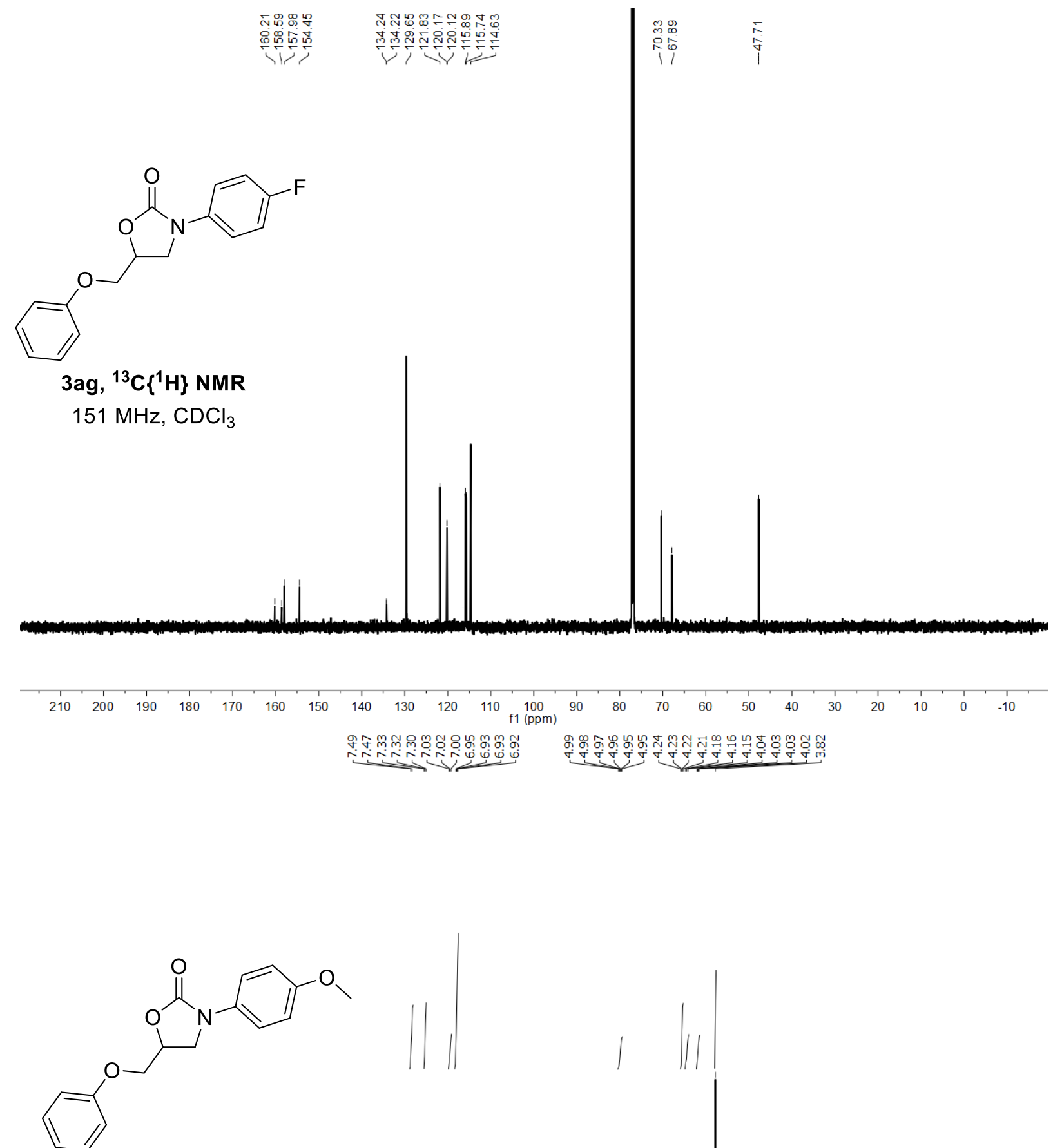

3ah, ${ }^{1} \mathrm{H}$ NMR

$600 \mathrm{MHz} \mathrm{CDCl}_{3}$

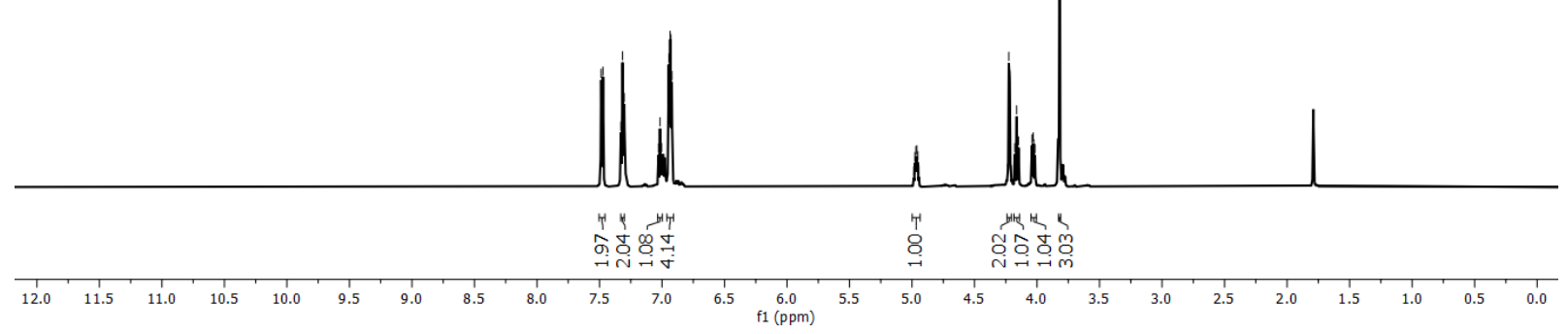



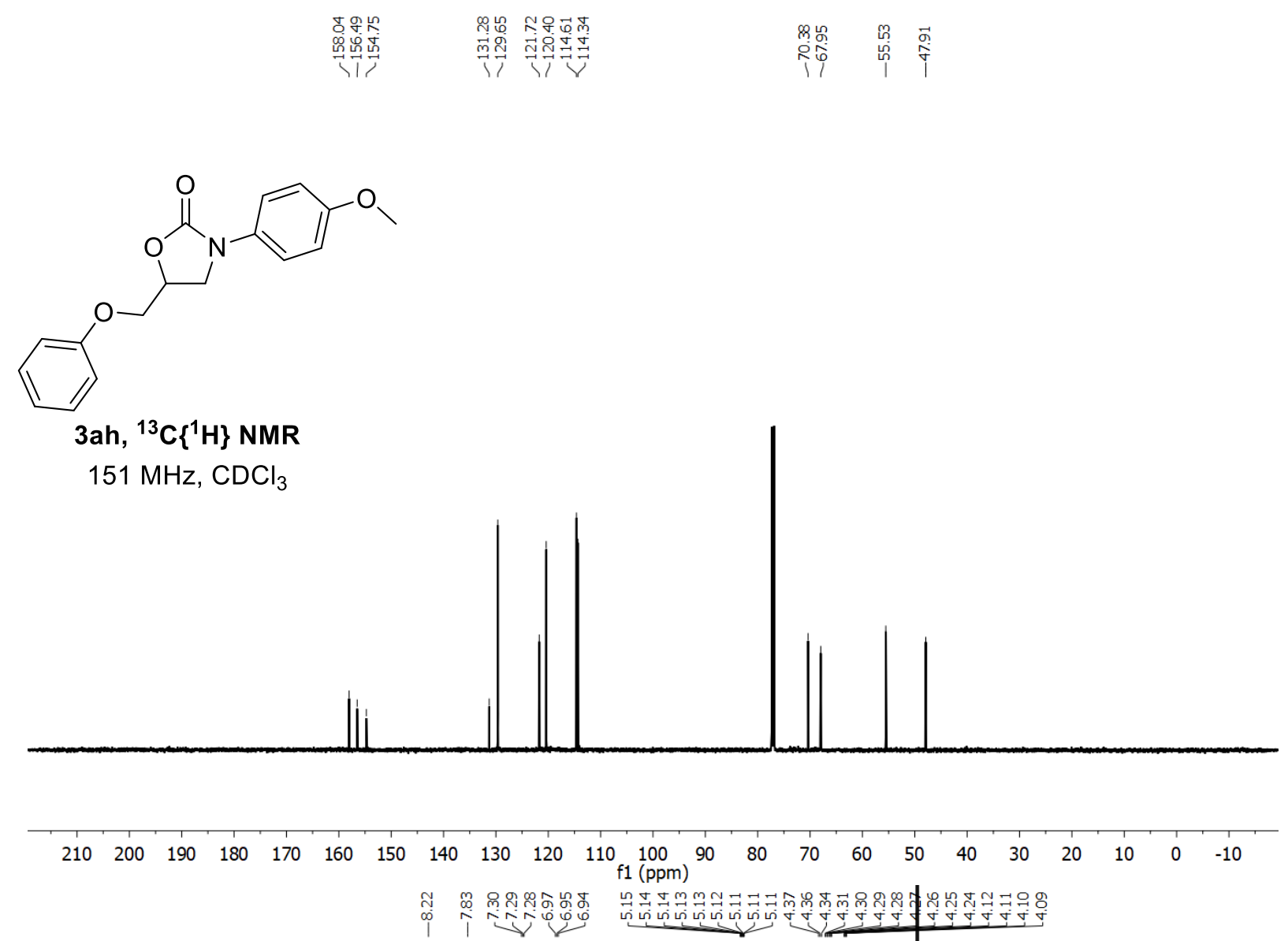<smiles>O=C1OC(COc2ccccc2)CN1c1cc(C(F)(F)F)cc(C(F)(F)F)c1</smiles>

3ai, ${ }^{1} \mathrm{H}$ NMR $600 \mathrm{MHz}$, DMSO- $d_{6}$

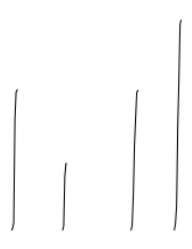

$$
\mid
$$
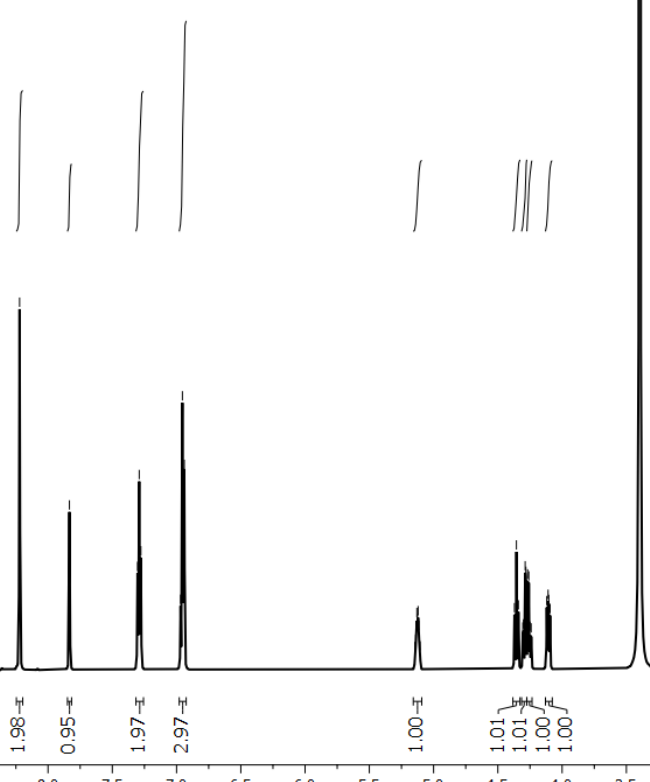

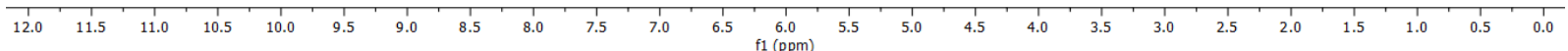



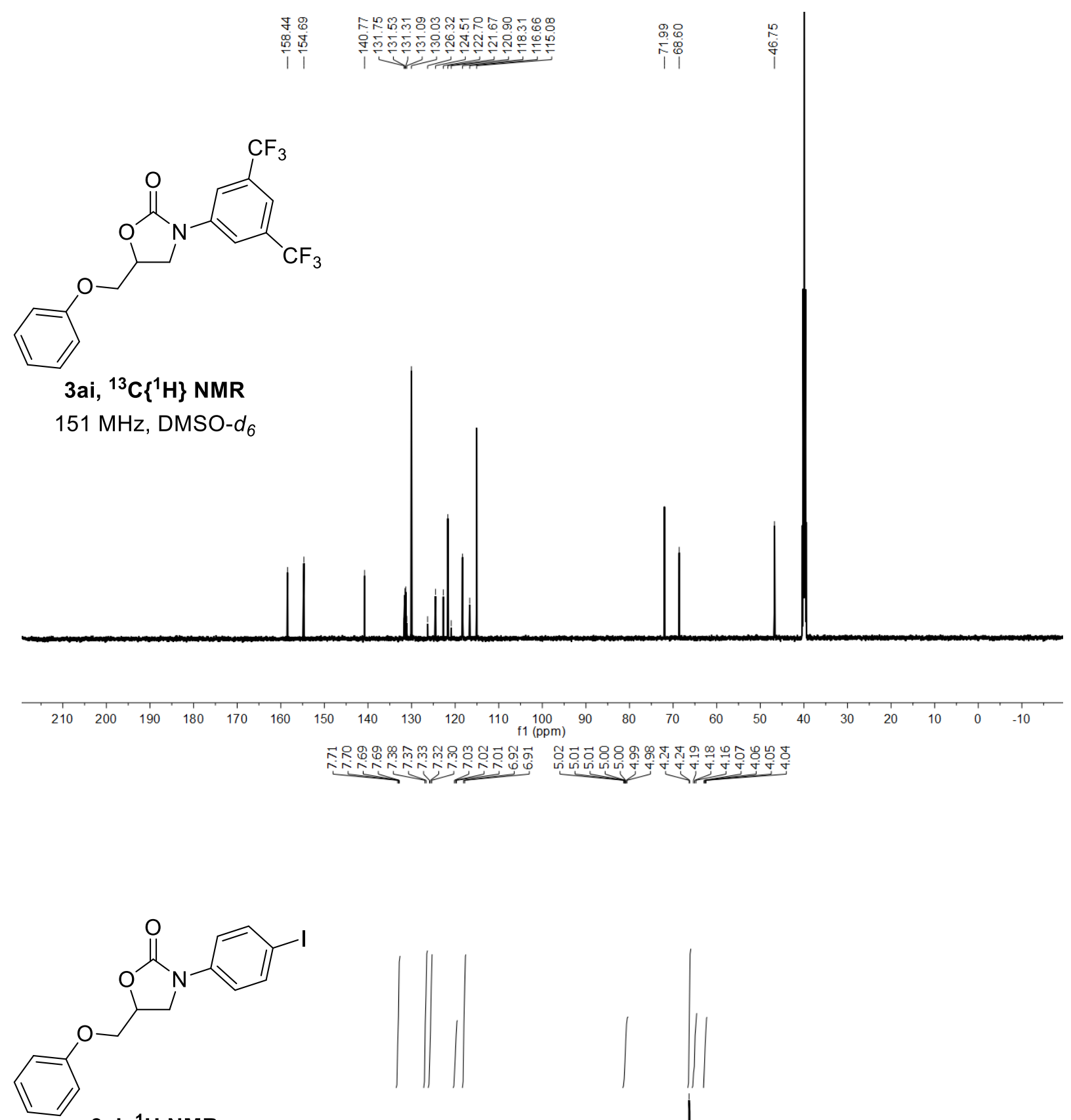

3aj, ${ }^{1} \mathrm{H}$ NMR

$600 \mathrm{MHz} \mathrm{CDCl}_{3}$

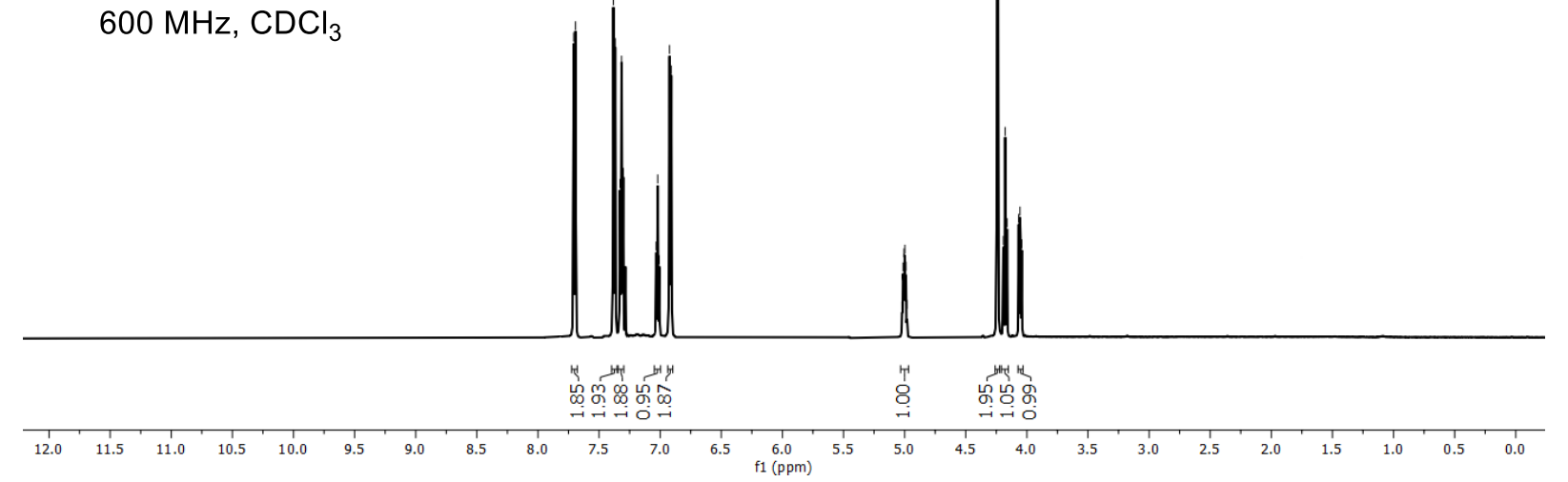



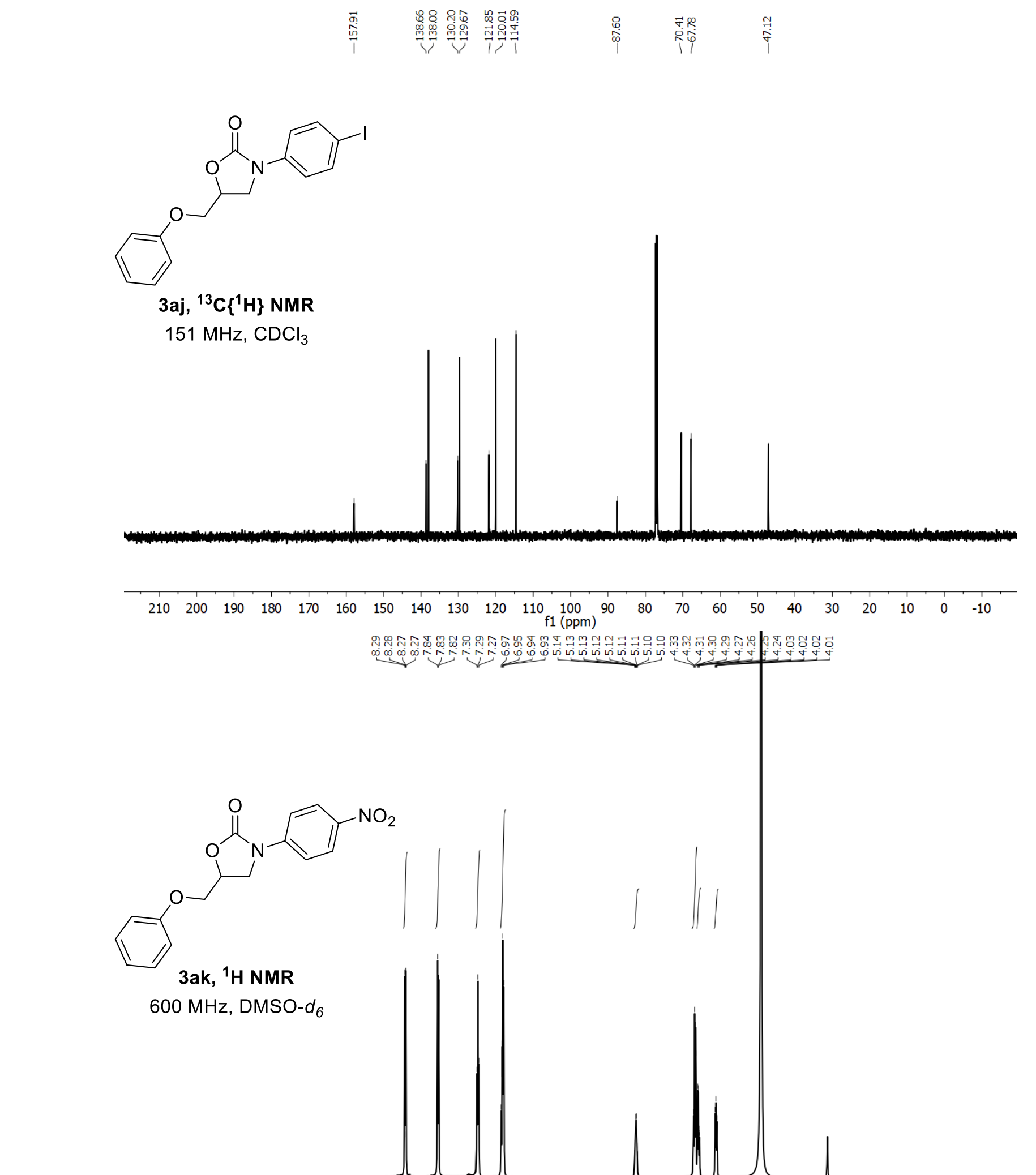

3ak, ${ }^{1} \mathrm{H}$ NMR

$600 \mathrm{MHz}, \mathrm{DMSO}-d_{6}$
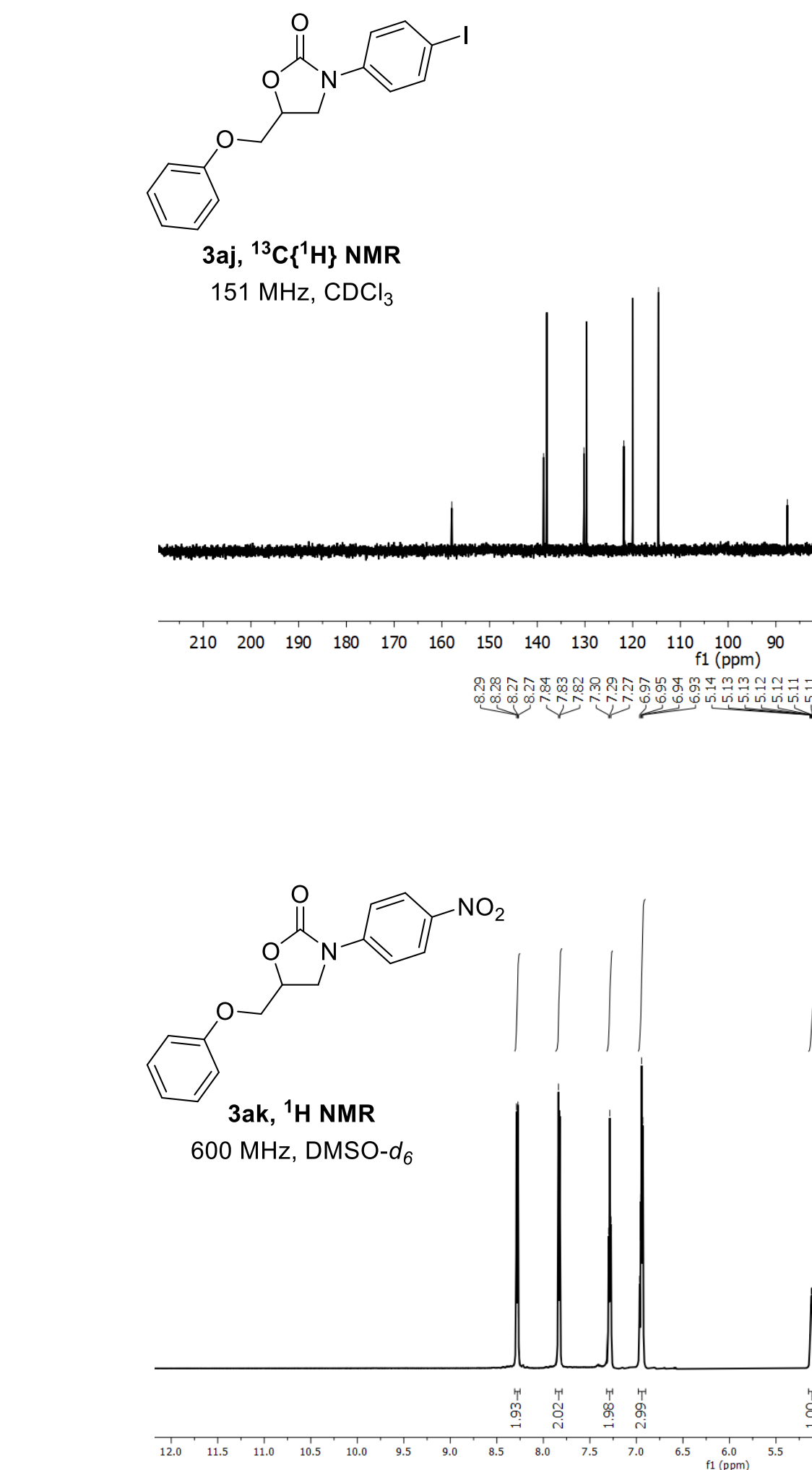

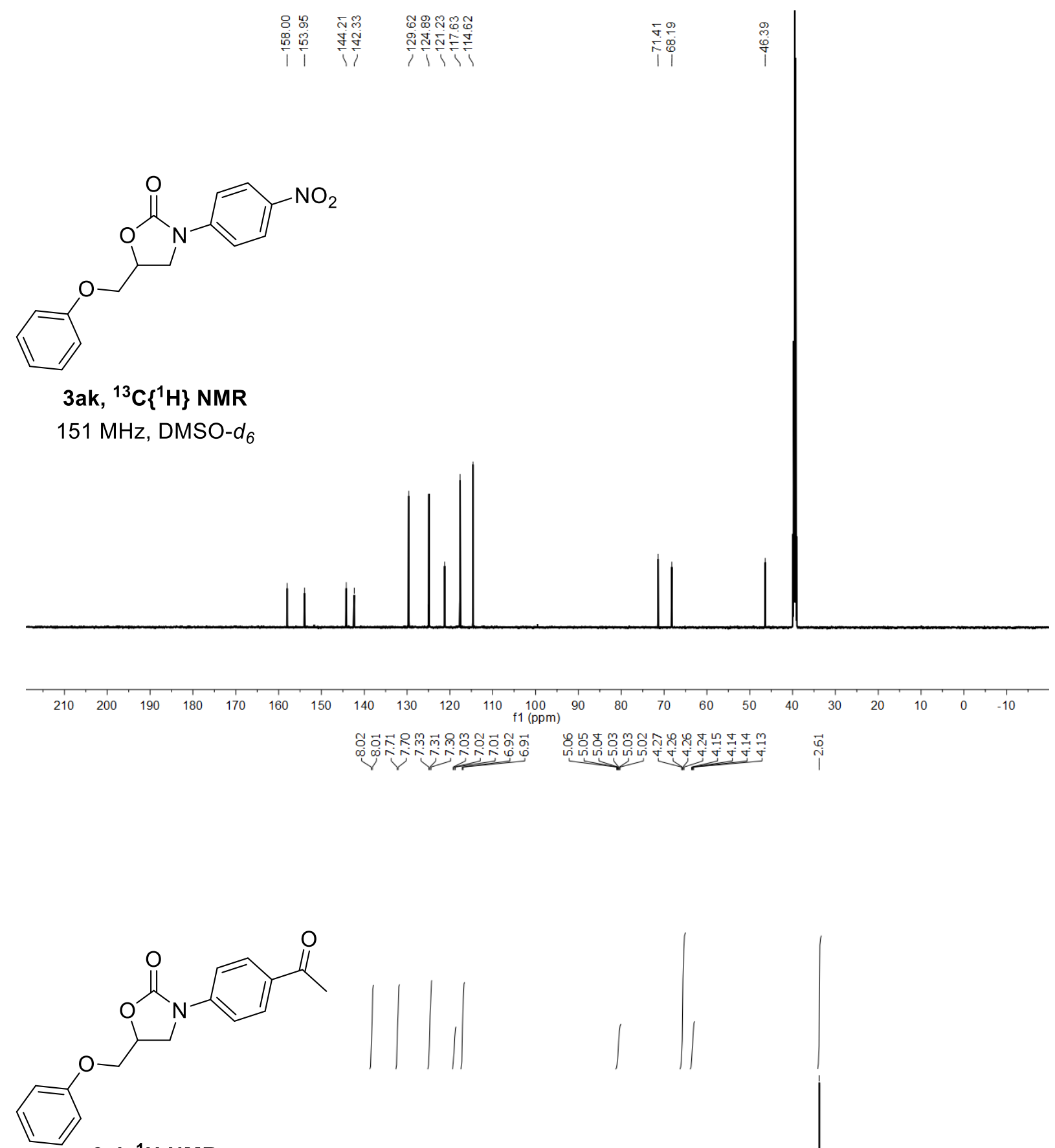

3al, ${ }^{1} \mathrm{H}$ NMR

$600 \mathrm{MHz} \mathrm{CDCl}_{3}$

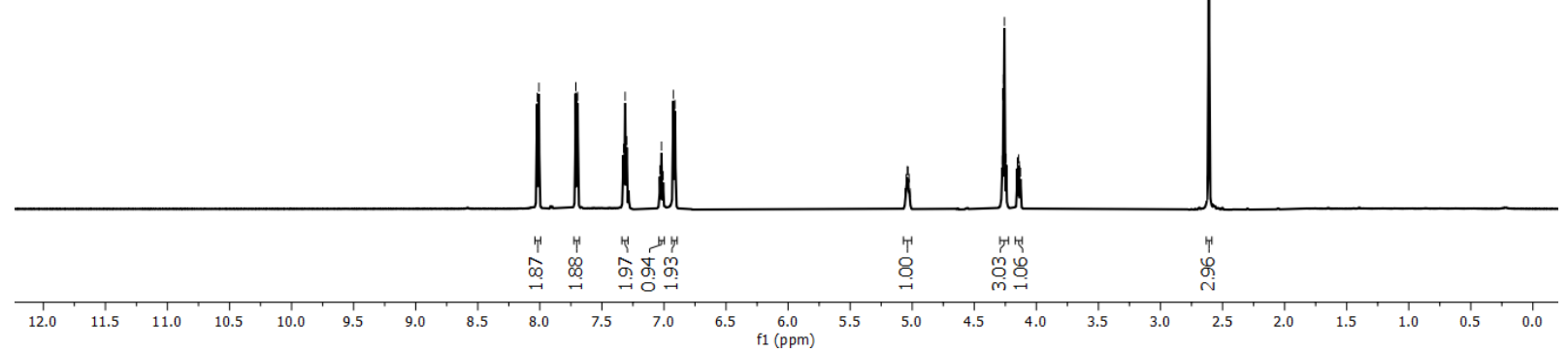



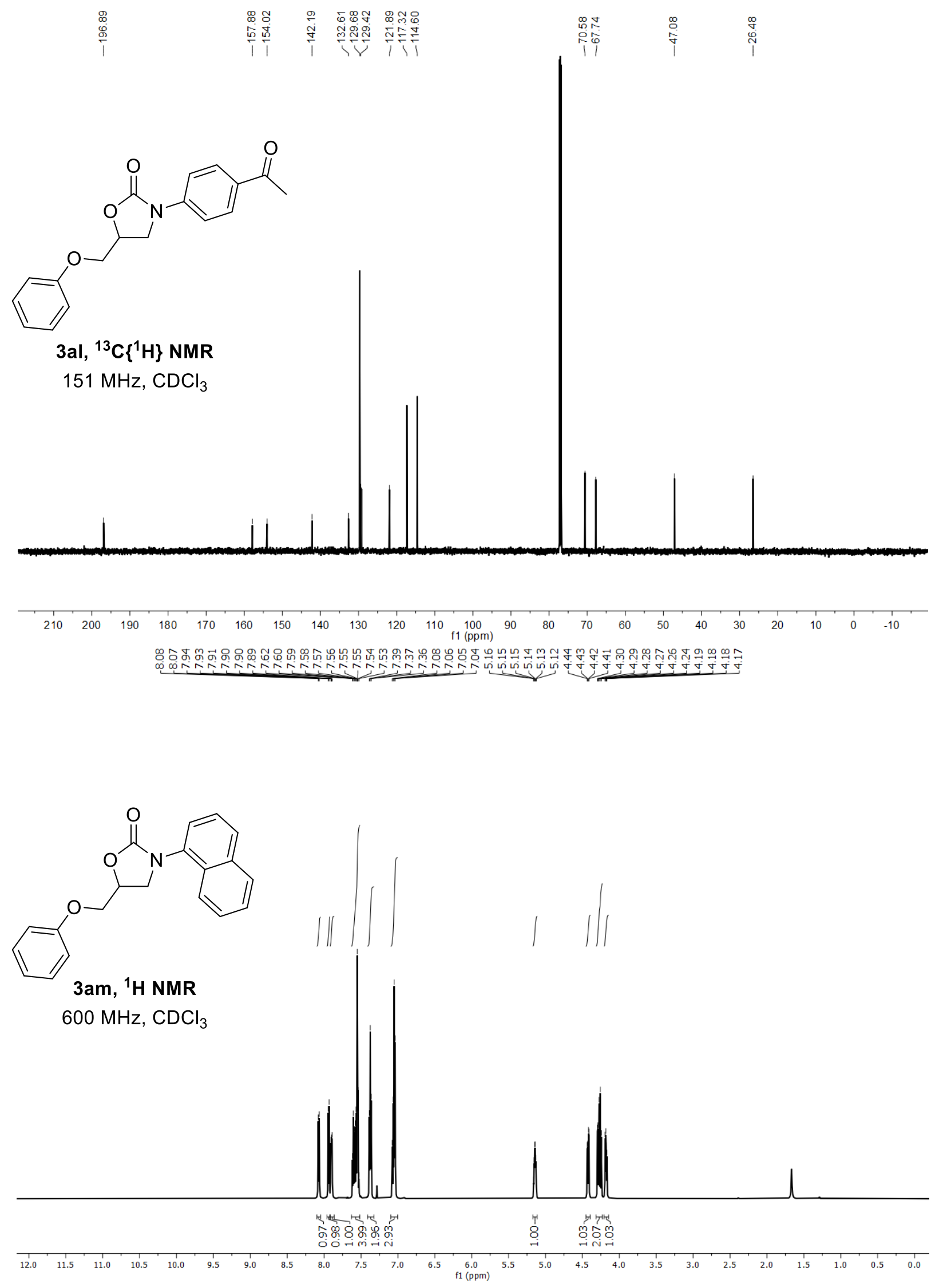


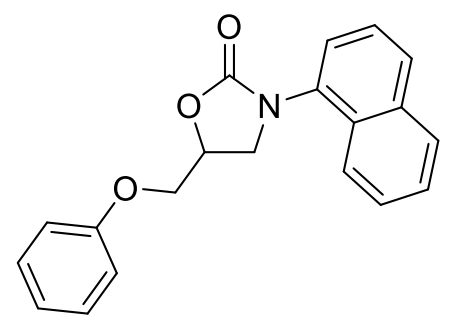

3am, ${ }^{13} \mathrm{C}\left\{{ }^{1} \mathrm{H}\right\}$ NMR

$151 \mathrm{MHz}, \mathrm{CDCl}_{3}$
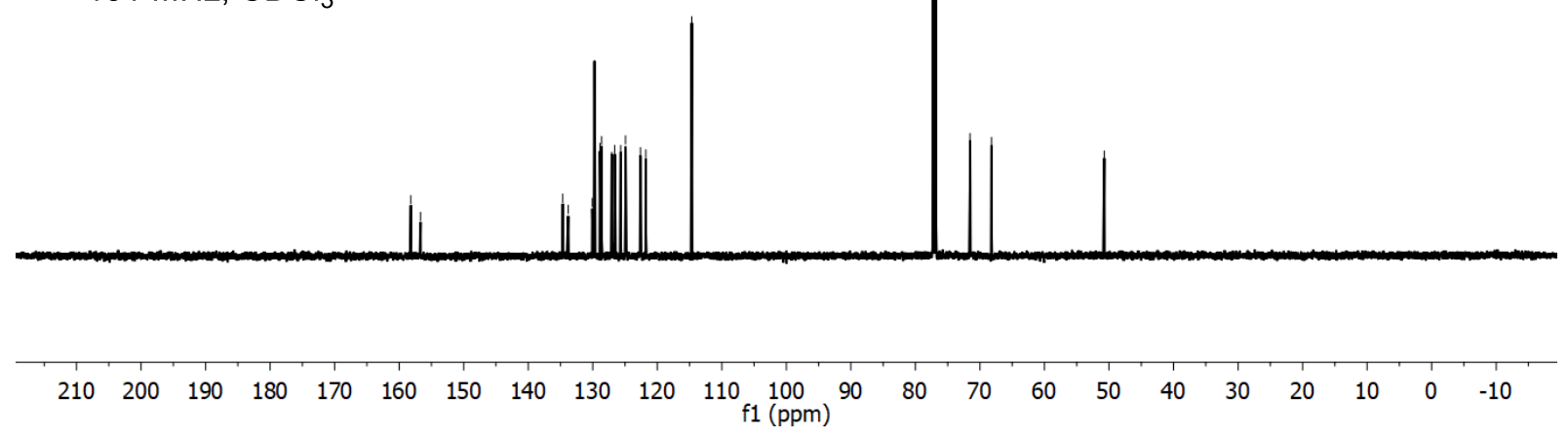

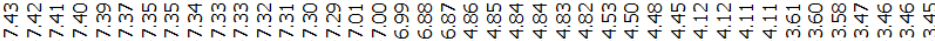<smiles>O=C1OC(COc2ccccc2)CN1Cc1ccccc1</smiles>

3an, ${ }^{1} \mathrm{H}$ NMR

$600 \mathrm{MHz}, \mathrm{CDCl}_{3}$

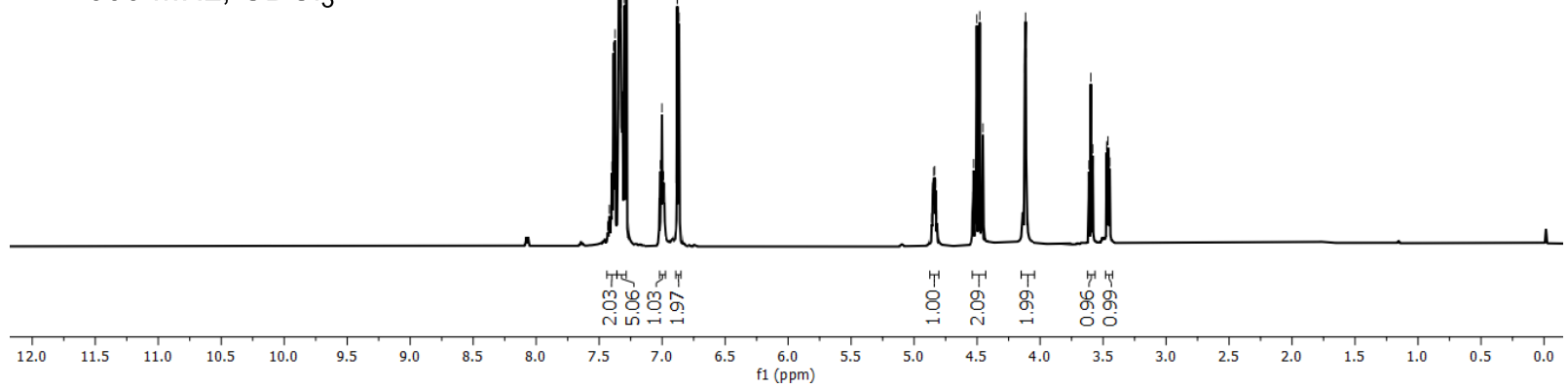



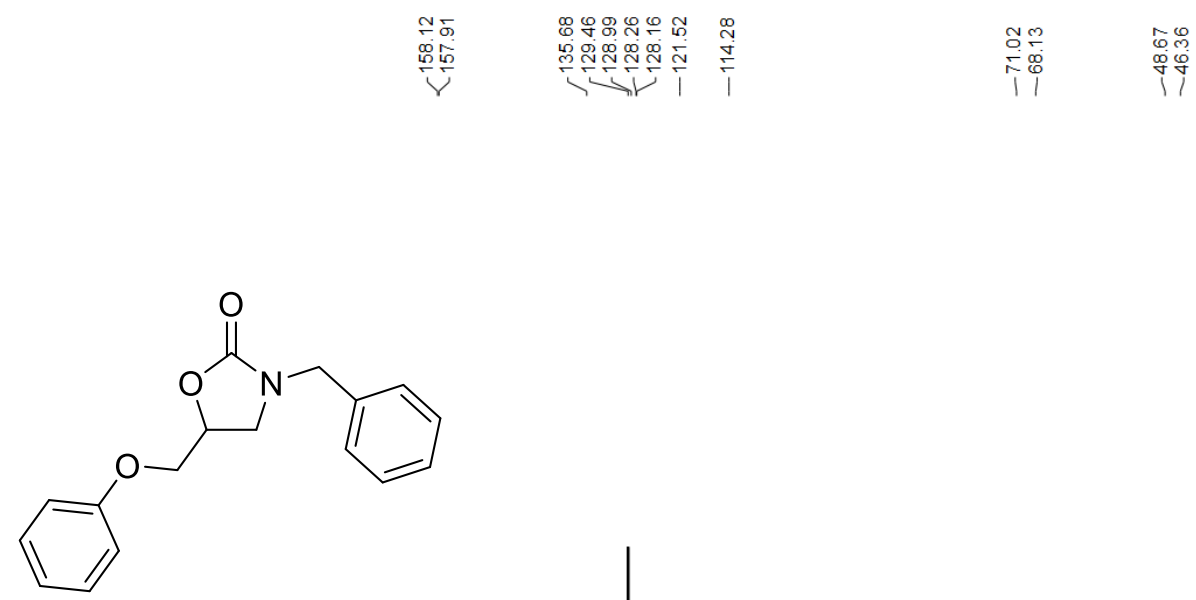

3an, ${ }^{13} \mathrm{C}\left\{{ }^{1} \mathrm{H}\right\}$ NMR

$151 \mathrm{MHz}, \mathrm{CDCl}_{3}$
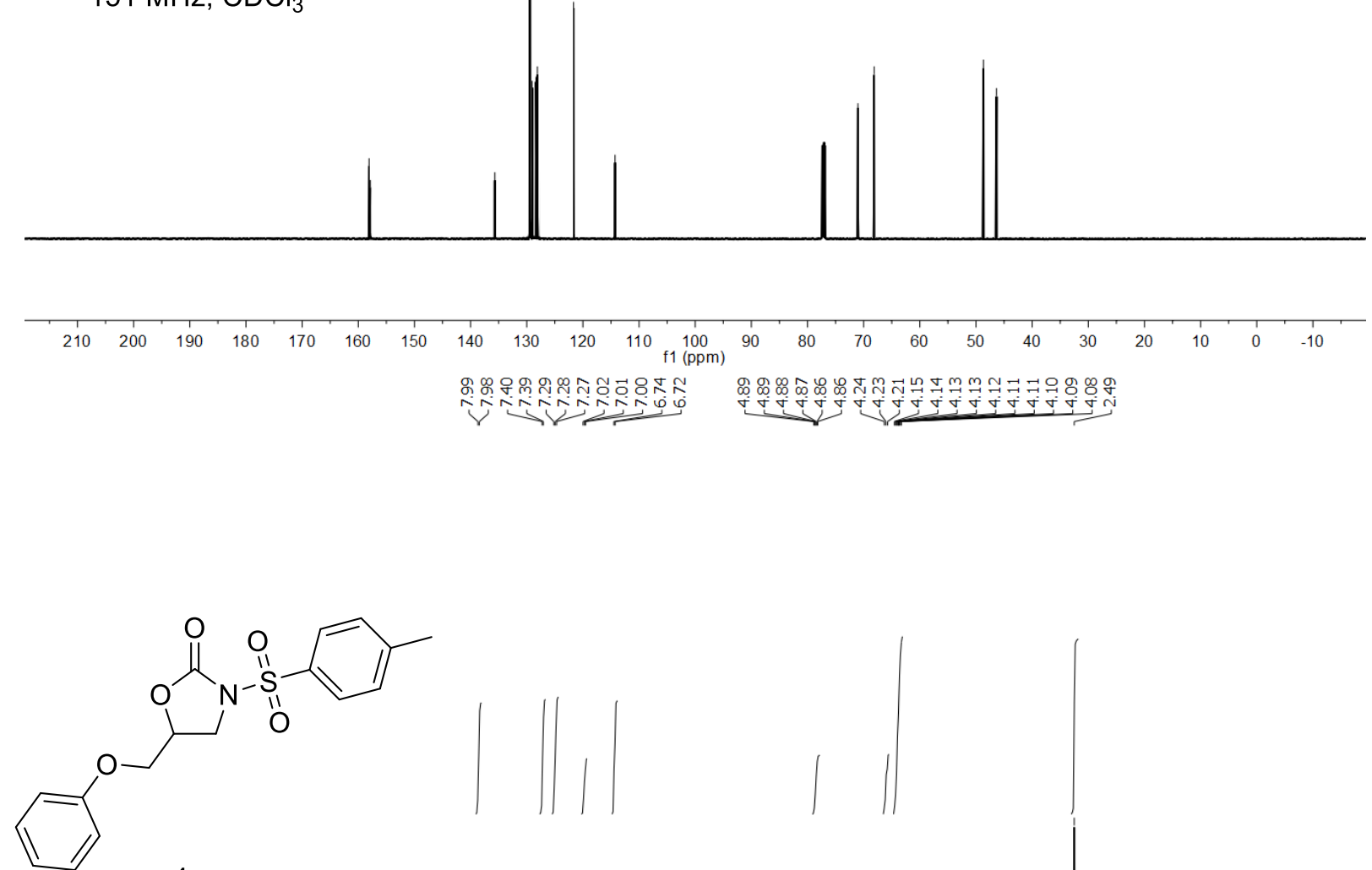

3ao, ${ }^{1} \mathrm{H}$ NMR

$600 \mathrm{MHz}, \mathrm{CDCl}_{3}$

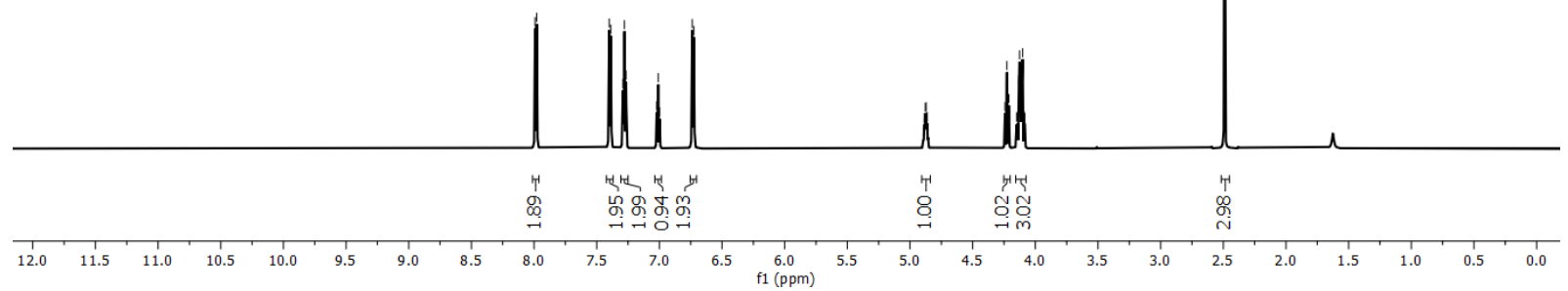




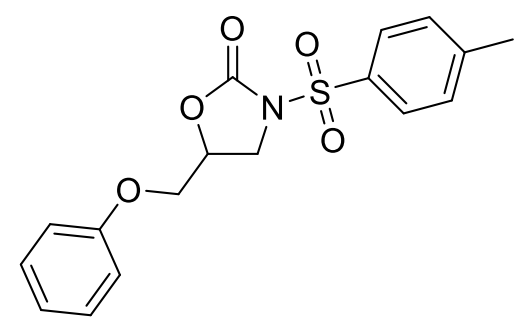

3ao, ${ }^{13} \mathrm{C}\left\{{ }^{1} \mathrm{H}\right\}$ NMR

$151 \mathrm{MHz}, \mathrm{CDCl}_{3}$

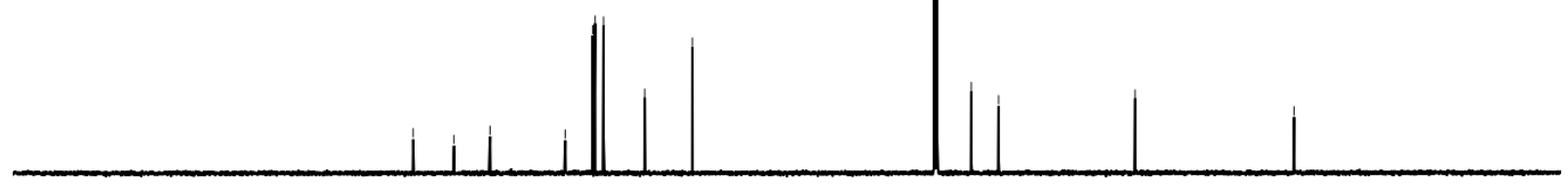

$\begin{array}{lllllllllllllllllllllllllll}210 & 200 & 190 & 180 & 170 & 160 & 150 & 140 & 130 & 120 & 110 & \begin{array}{c}100 \\ \mathrm{f} 1(\mathrm{ppm})\end{array} & 90 & 80 & 70 & 60 & 50 & 40 & 30 & 20 & 10 & 0 & -10 & \end{array}$

мैmp

作

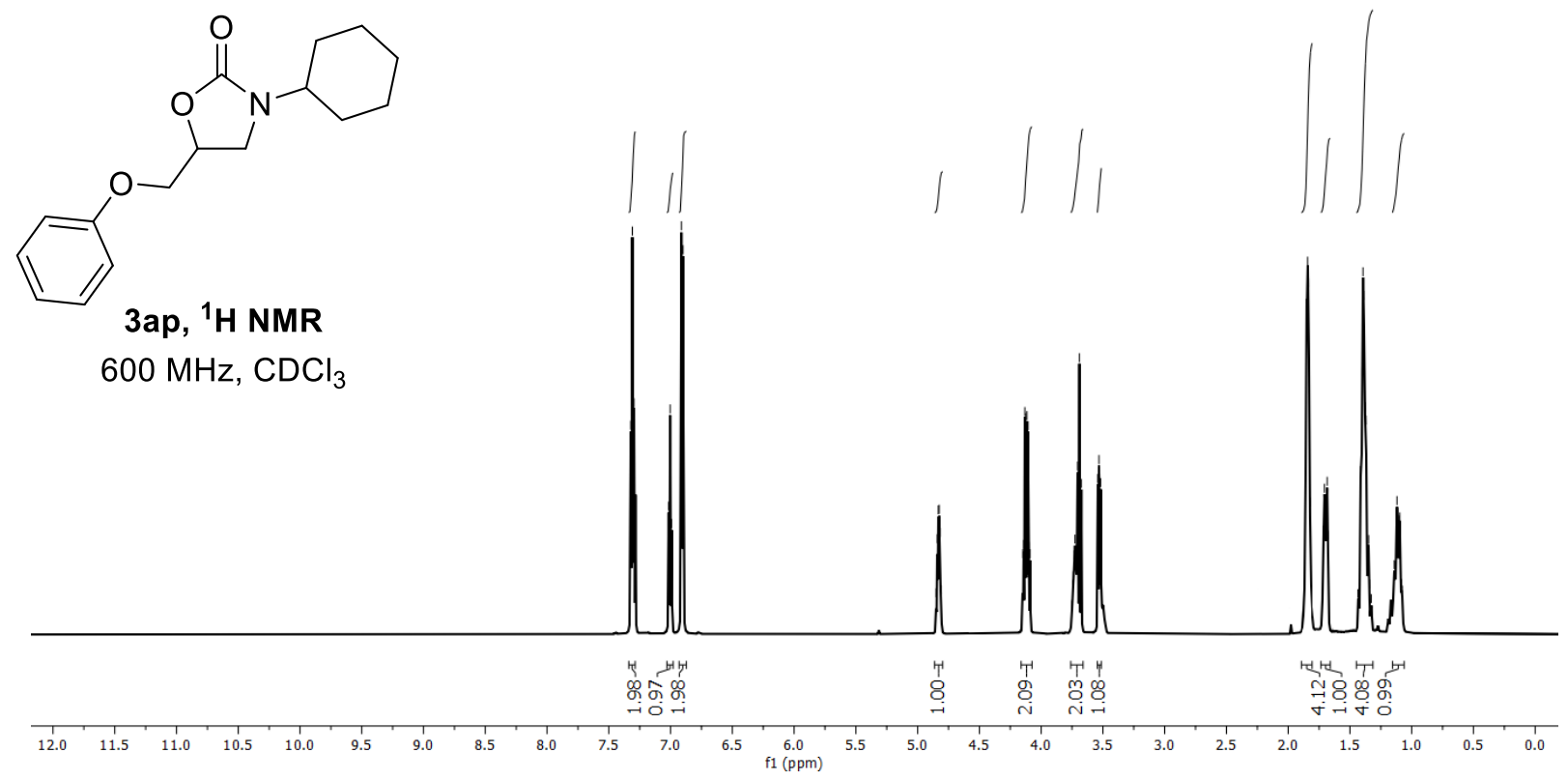



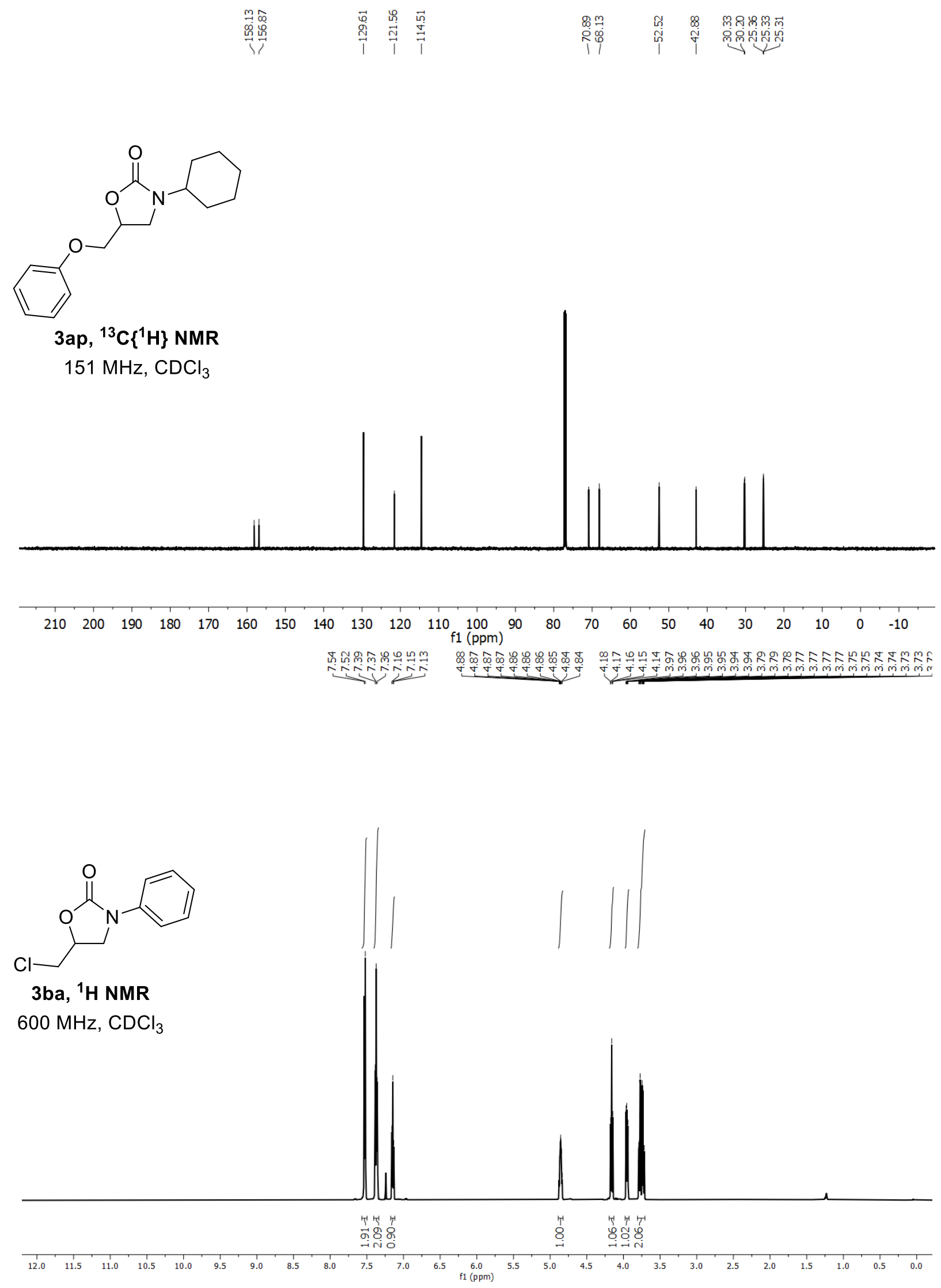
<smiles>O=C1OC(CCl)CN1c1ccccc1</smiles>

3ba, ${ }^{13} \mathrm{C}\left\{{ }^{1} \mathrm{H}\right\}$ NMR

$151 \mathrm{MHz}, \mathrm{CDCl}_{3}$
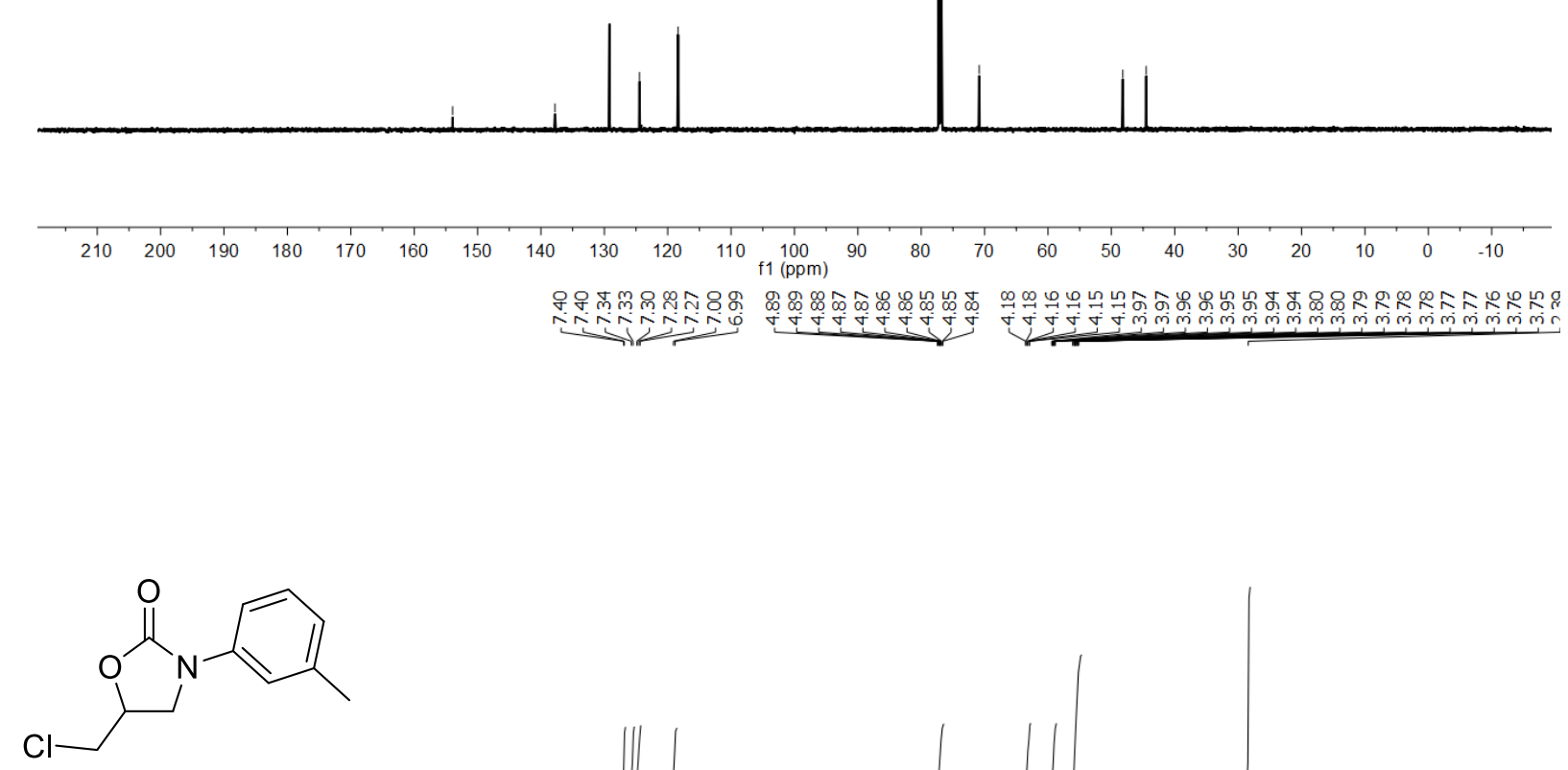

3bc, ${ }^{1} \mathrm{H}$ NMR

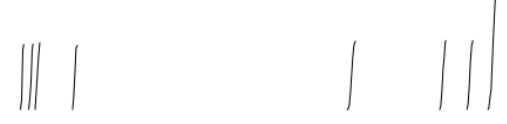

$600 \mathrm{MHz}, \mathrm{CDCl}_{3}$

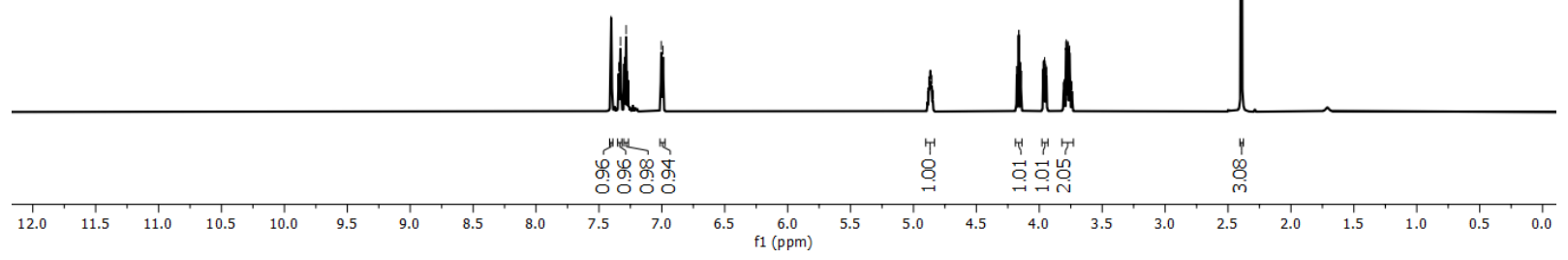



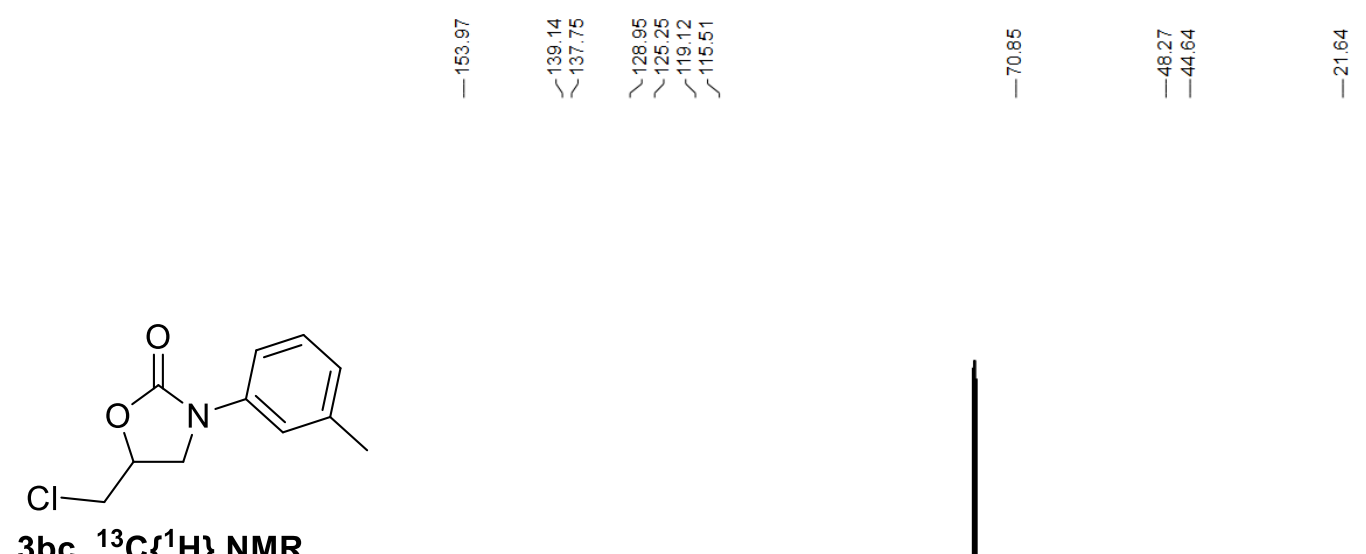

3bc, ${ }^{13} \mathrm{C}\left\{{ }^{1} \mathrm{H}\right\}$ NMR

$151 \mathrm{MHz}, \mathrm{CDCl}_{3}$
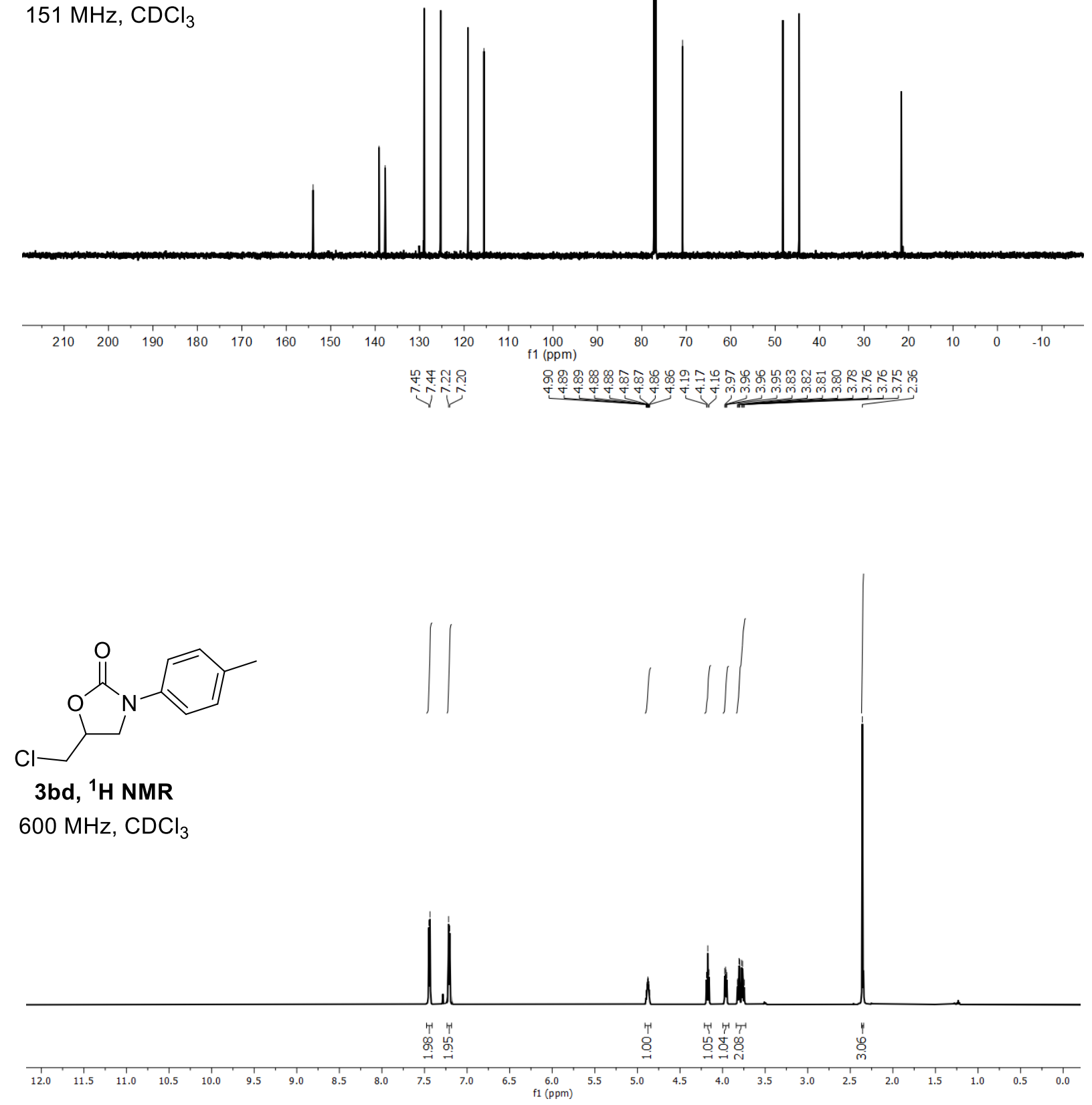


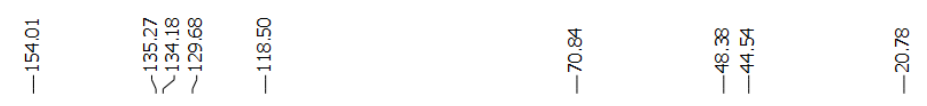

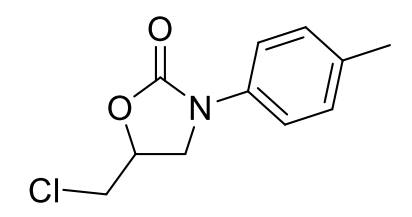

3bd, ${ }^{13} \mathrm{C}\left\{{ }^{1} \mathrm{H}\right\}$ NMR

$151 \mathrm{MHz}, \mathrm{CDCl}_{3}$
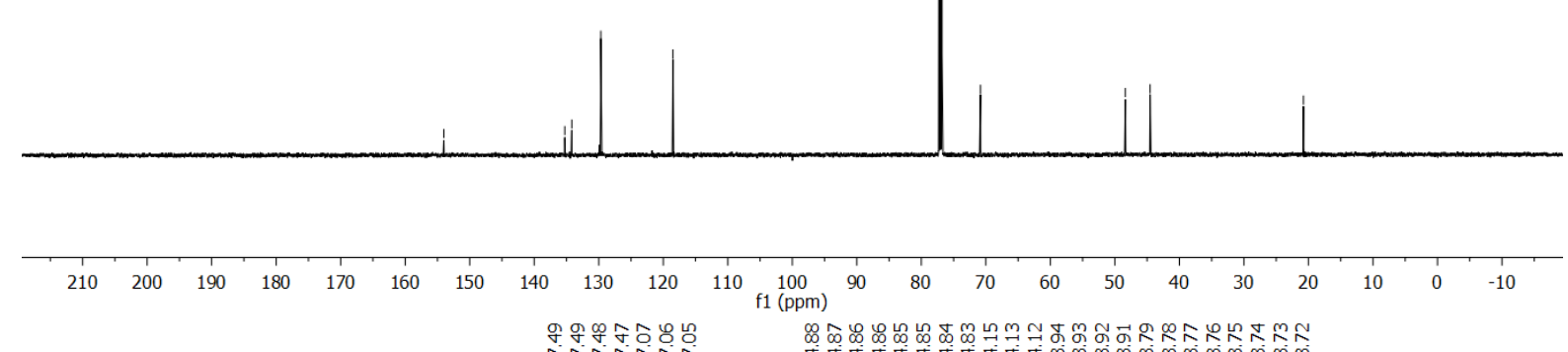<smiles>O=C1OC(CCl)CN1c1ccc(F)cc1</smiles>

3bg, ${ }^{1} \mathrm{H}$ NMR

$600 \mathrm{MHz}^{\mathrm{CDCl}_{3}}$

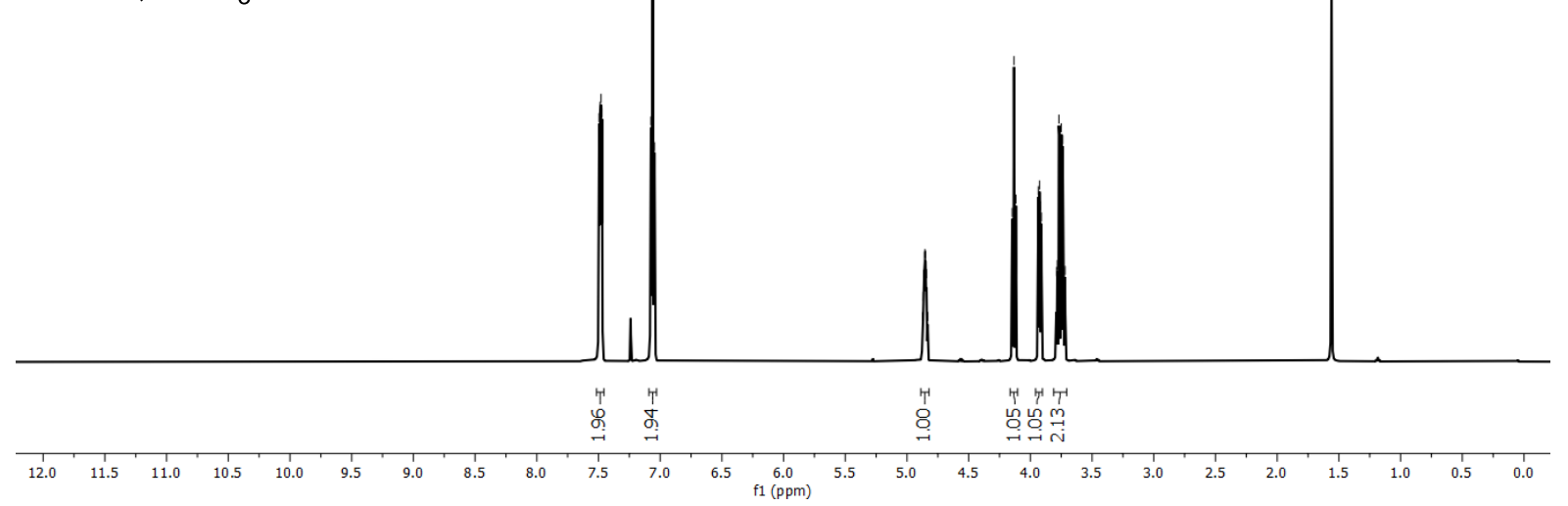



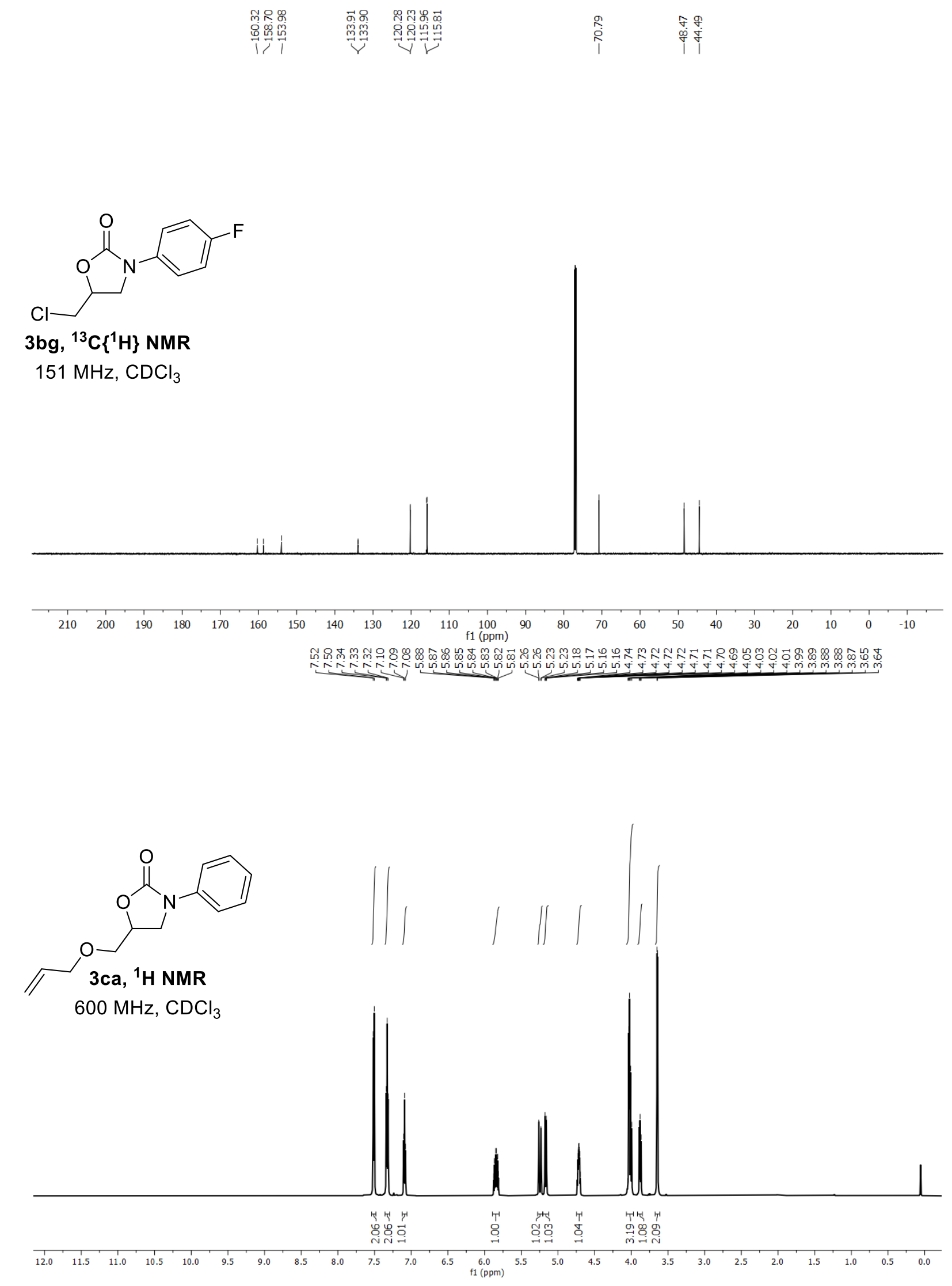

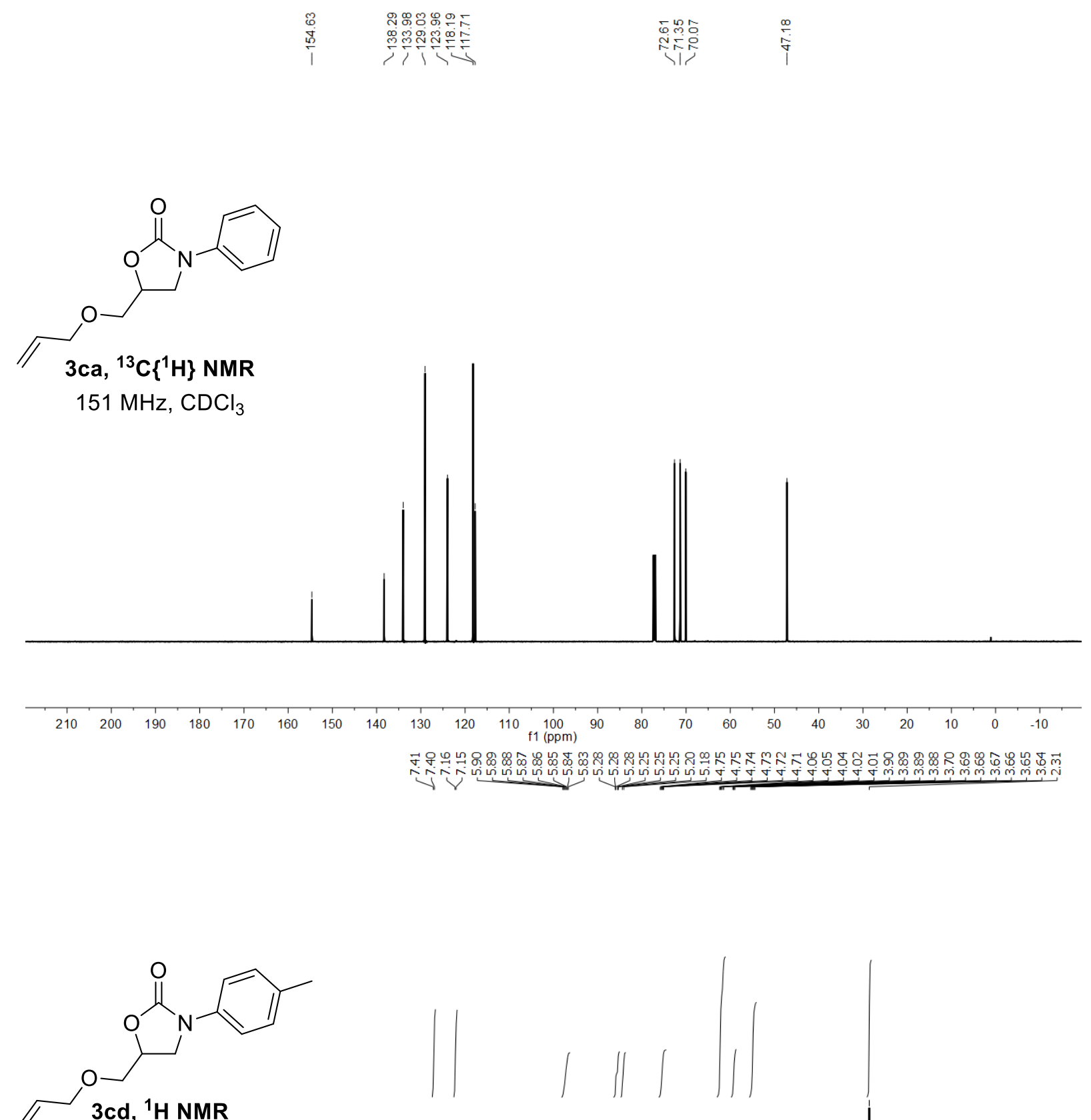

$600 \mathrm{MHz}, \mathrm{CDCl}_{3}$

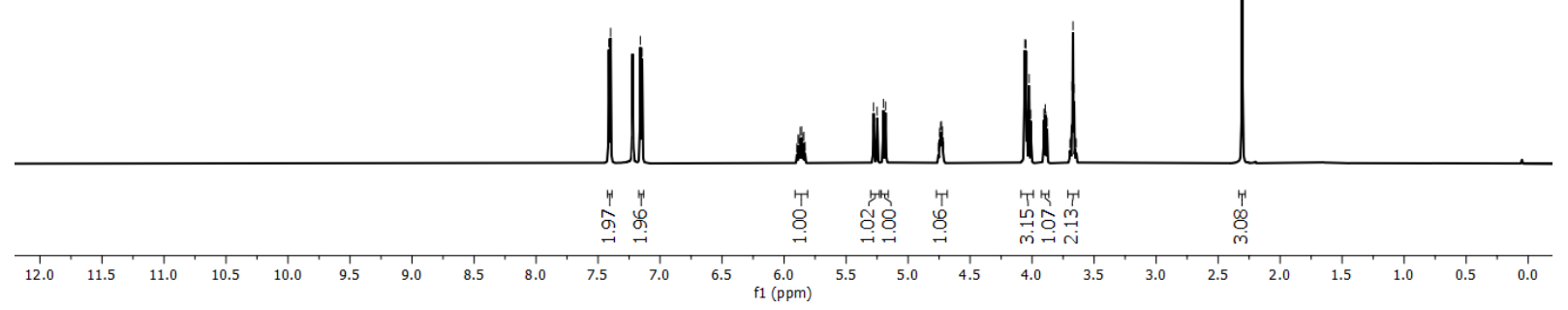



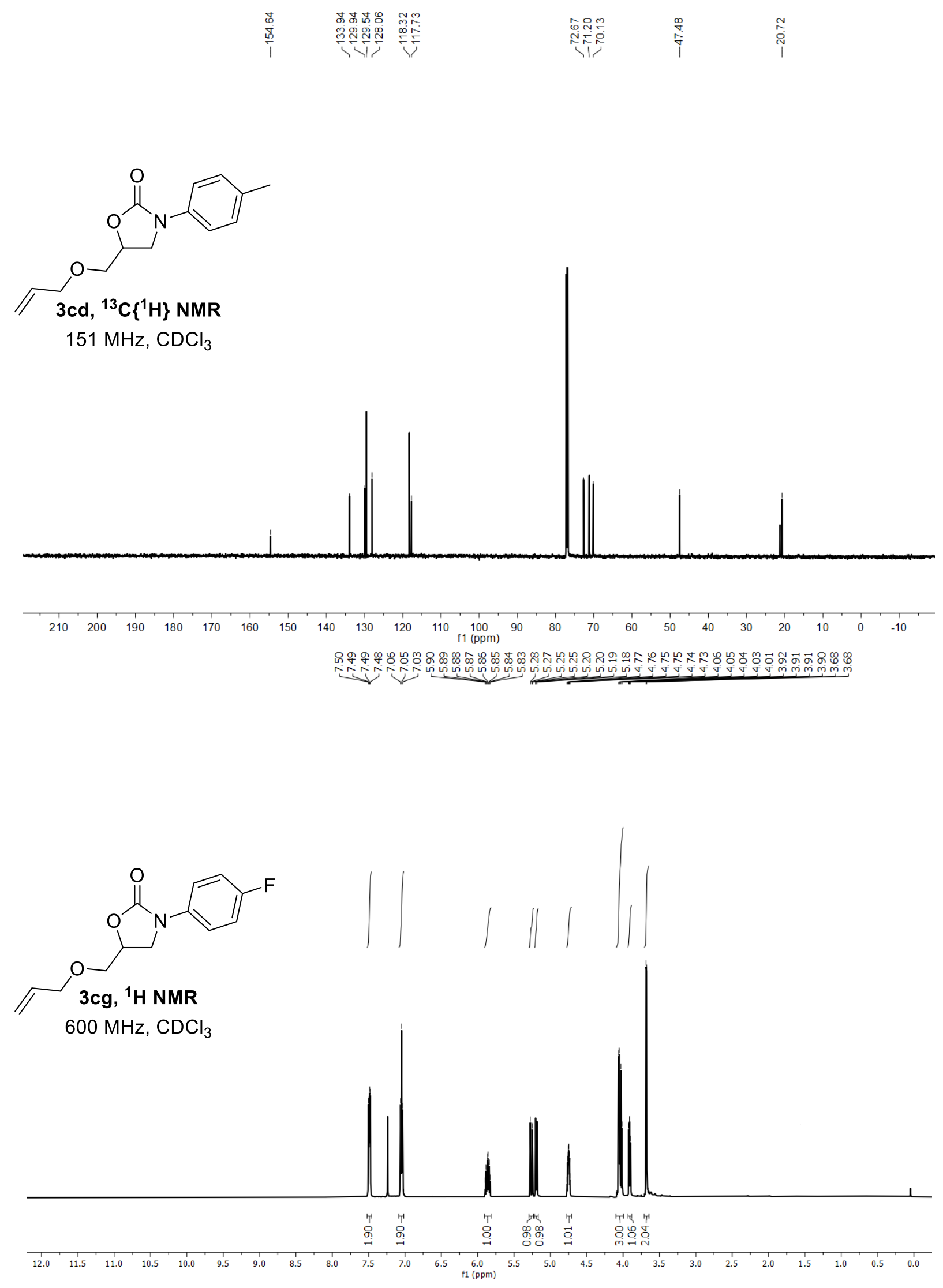

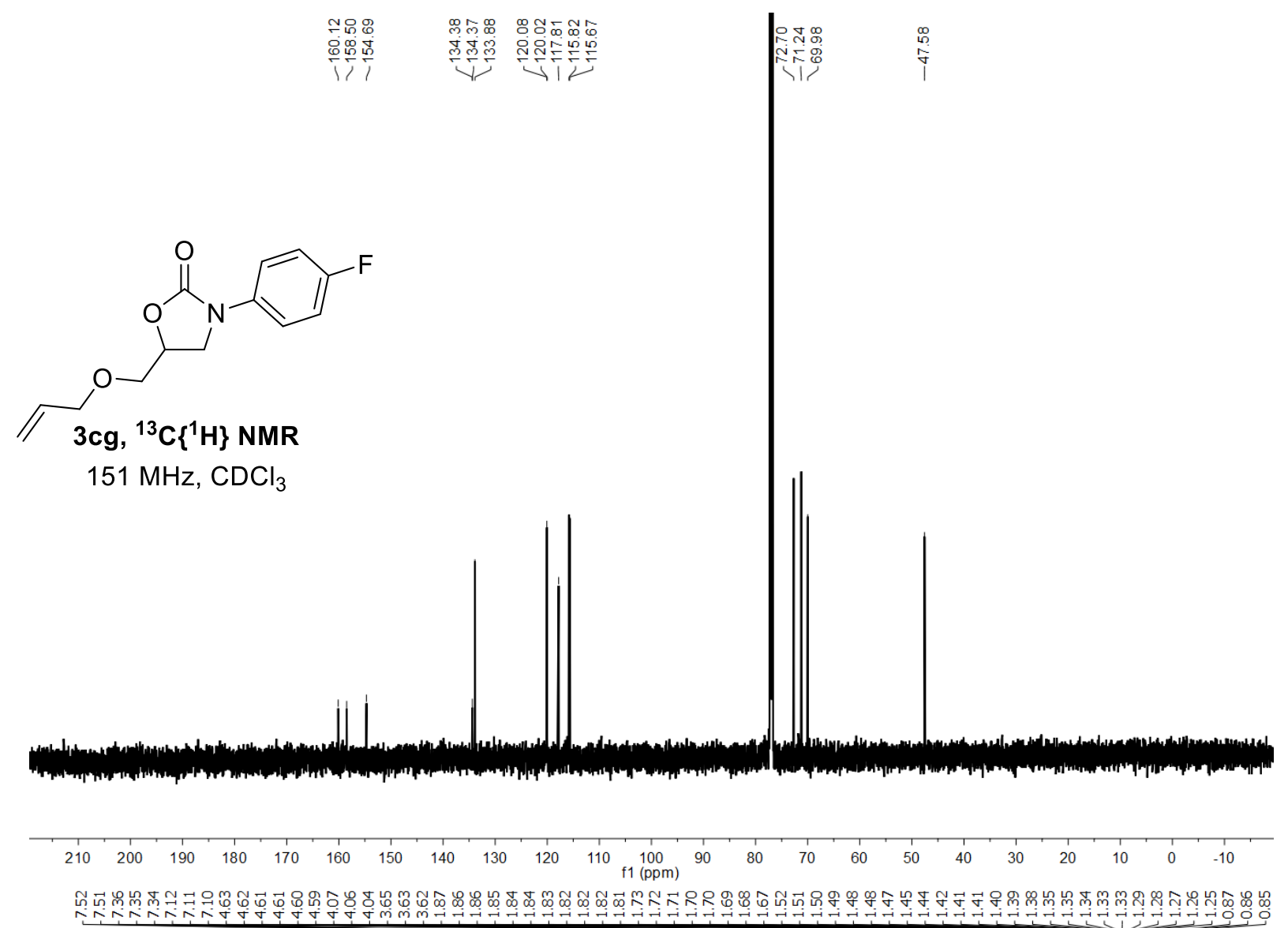<smiles>[15CH2]CC1CN(c2ccccc2)C(=O)O1</smiles>

3da, ${ }^{1} \mathrm{H}$ NMR

$600 \mathrm{MHz}, \mathrm{CDCl}_{3}$

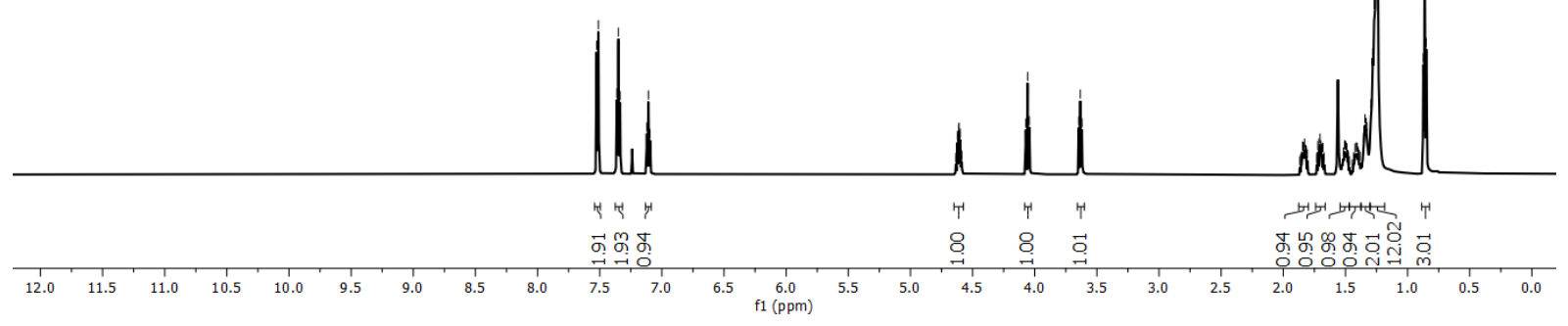



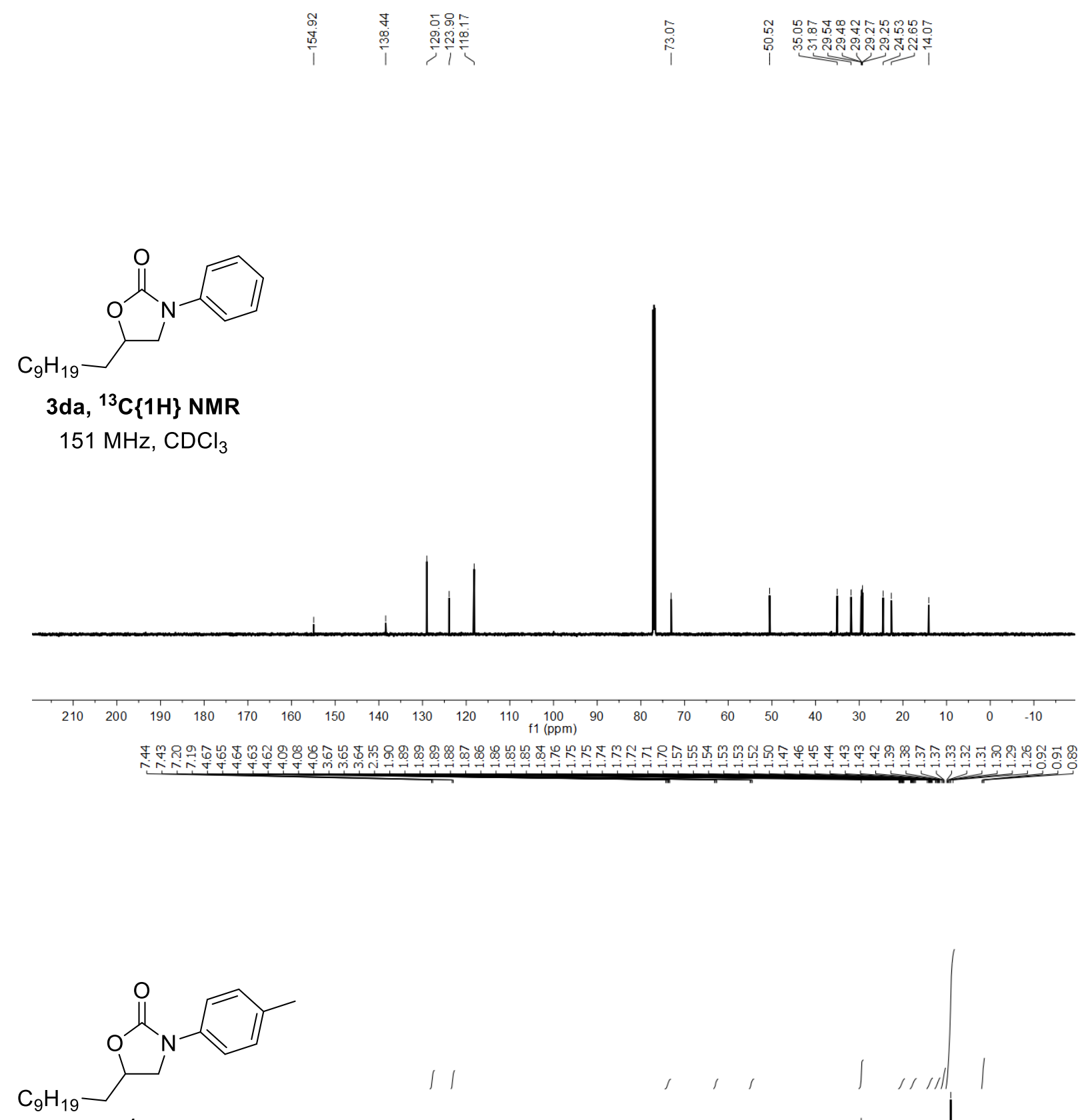

3dd, ${ }^{1} \mathrm{H}$ NMR

$600 \mathrm{MHz}, \mathrm{CDCl}_{3}$

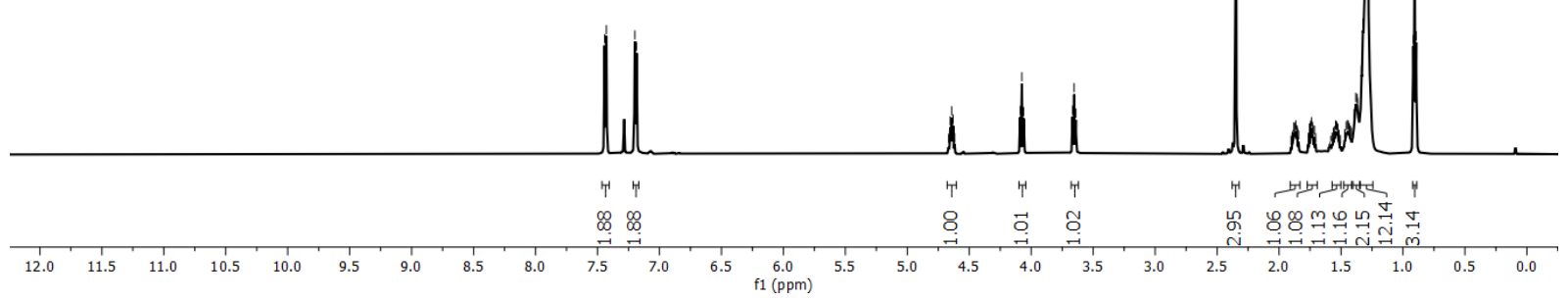



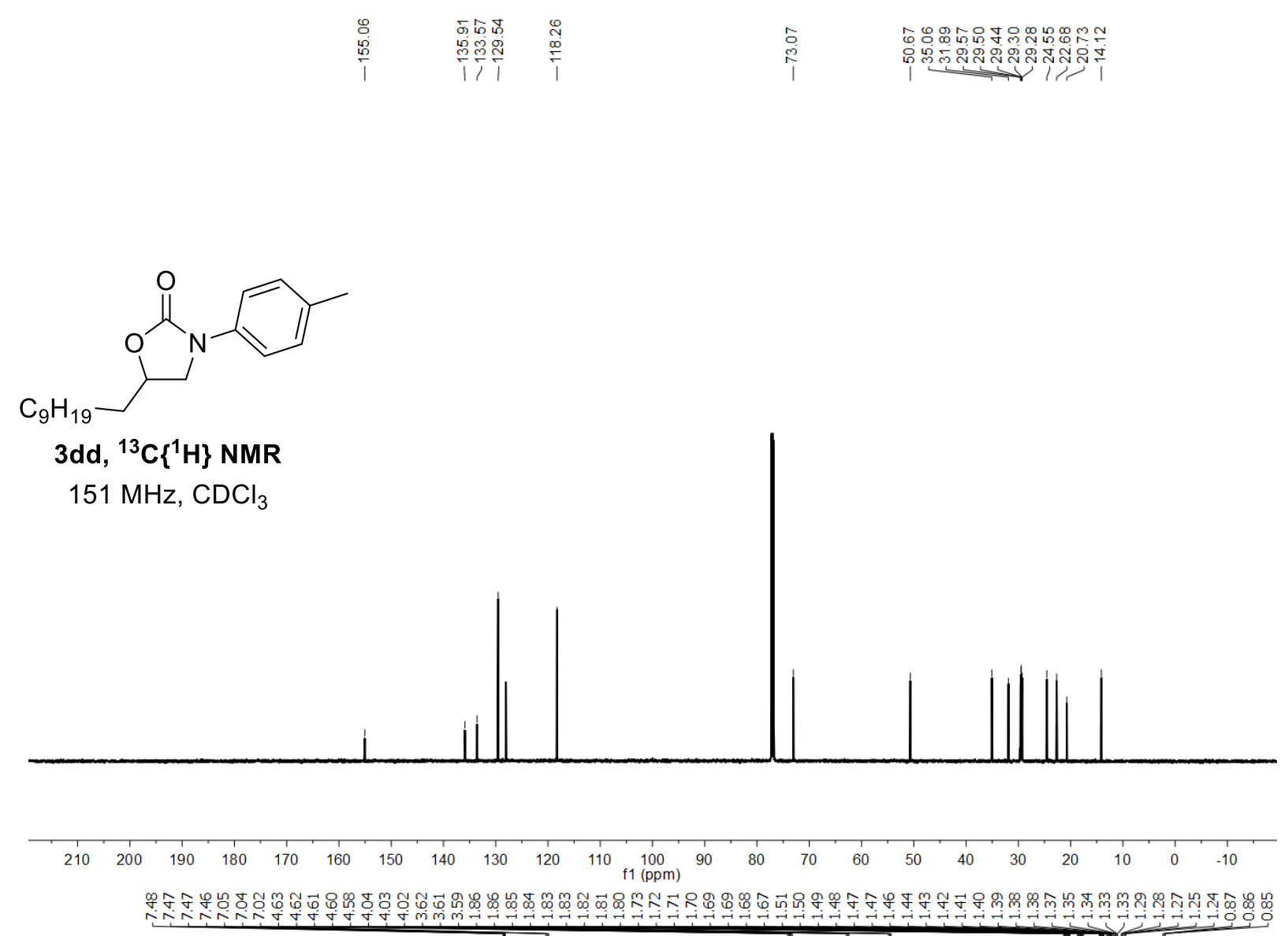<smiles>O=C1OC(C[125I])CN1c1ccc(F)cc1</smiles>

$3 \mathrm{dg},{ }^{1} \mathrm{H}$ NMR

$600 \mathrm{MHz}, \mathrm{CDCl}_{3}$

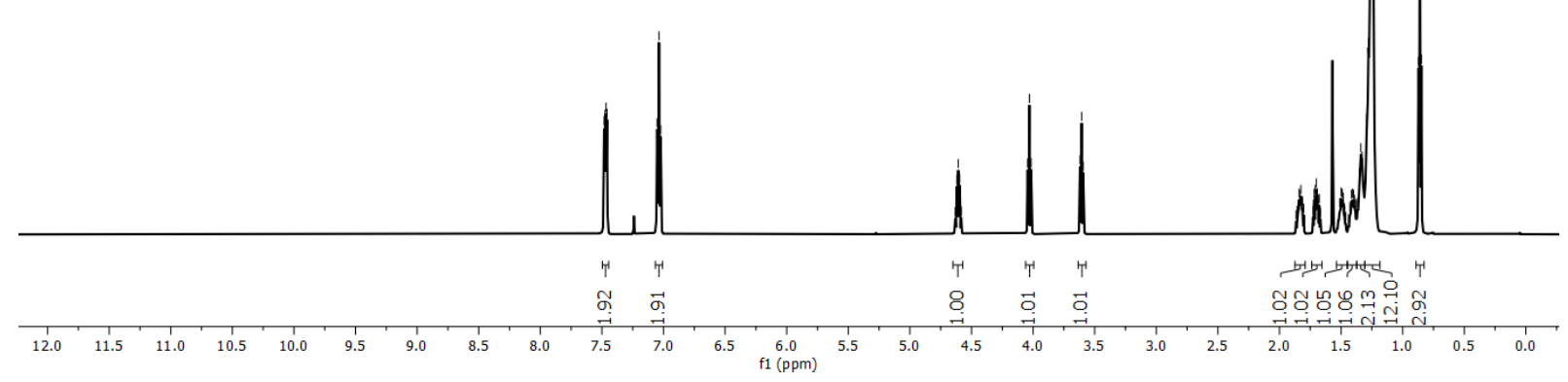



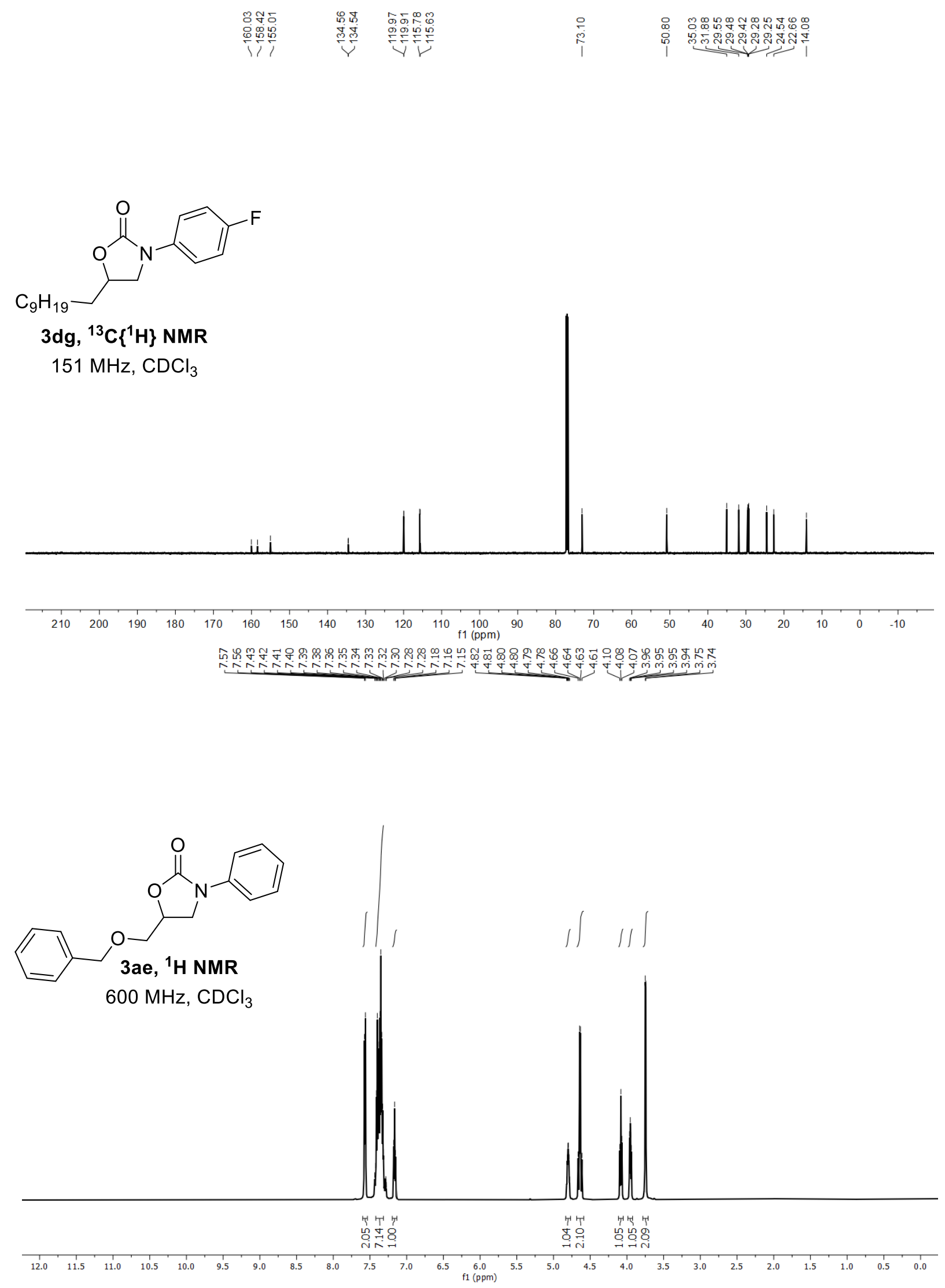

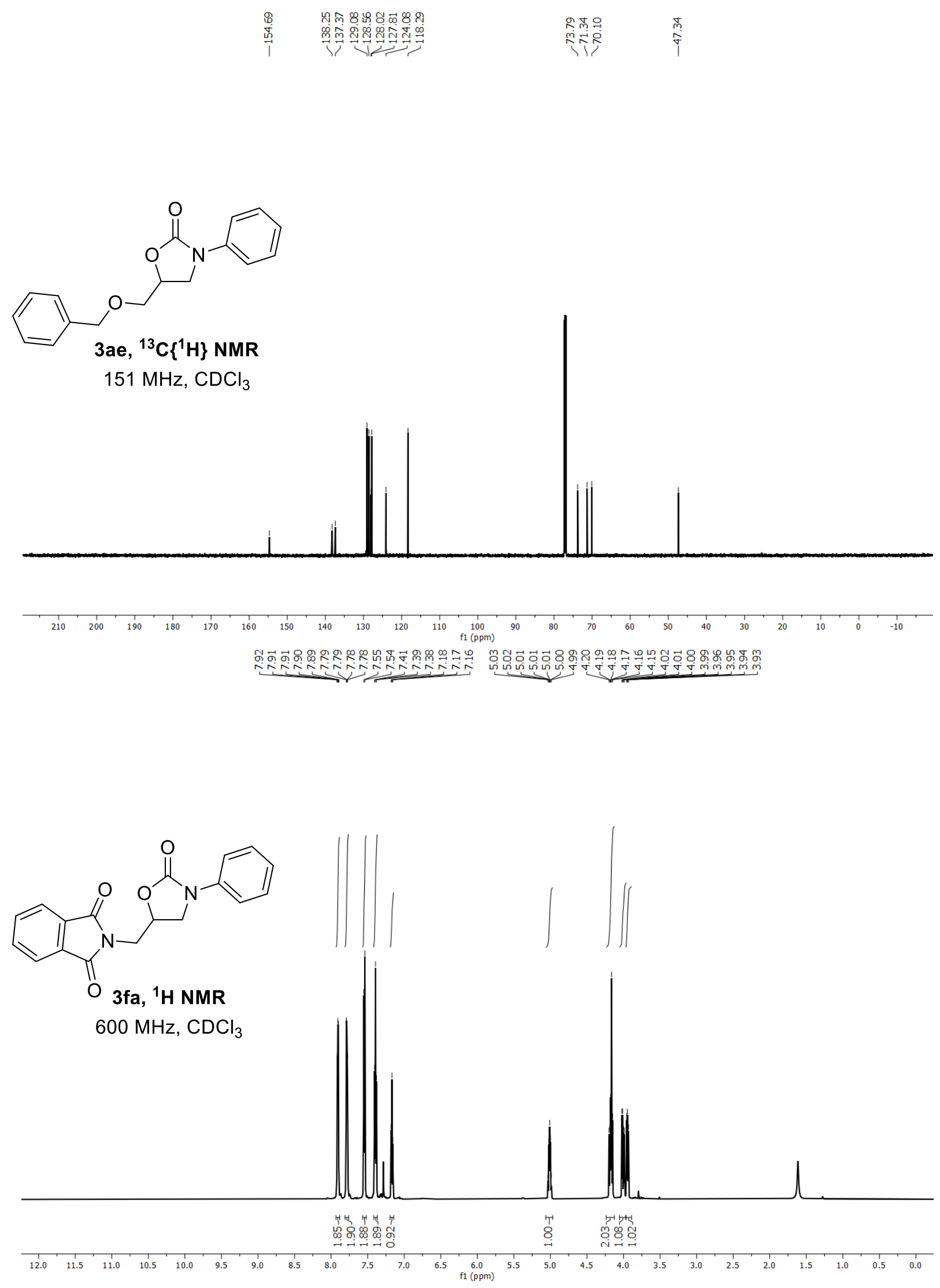

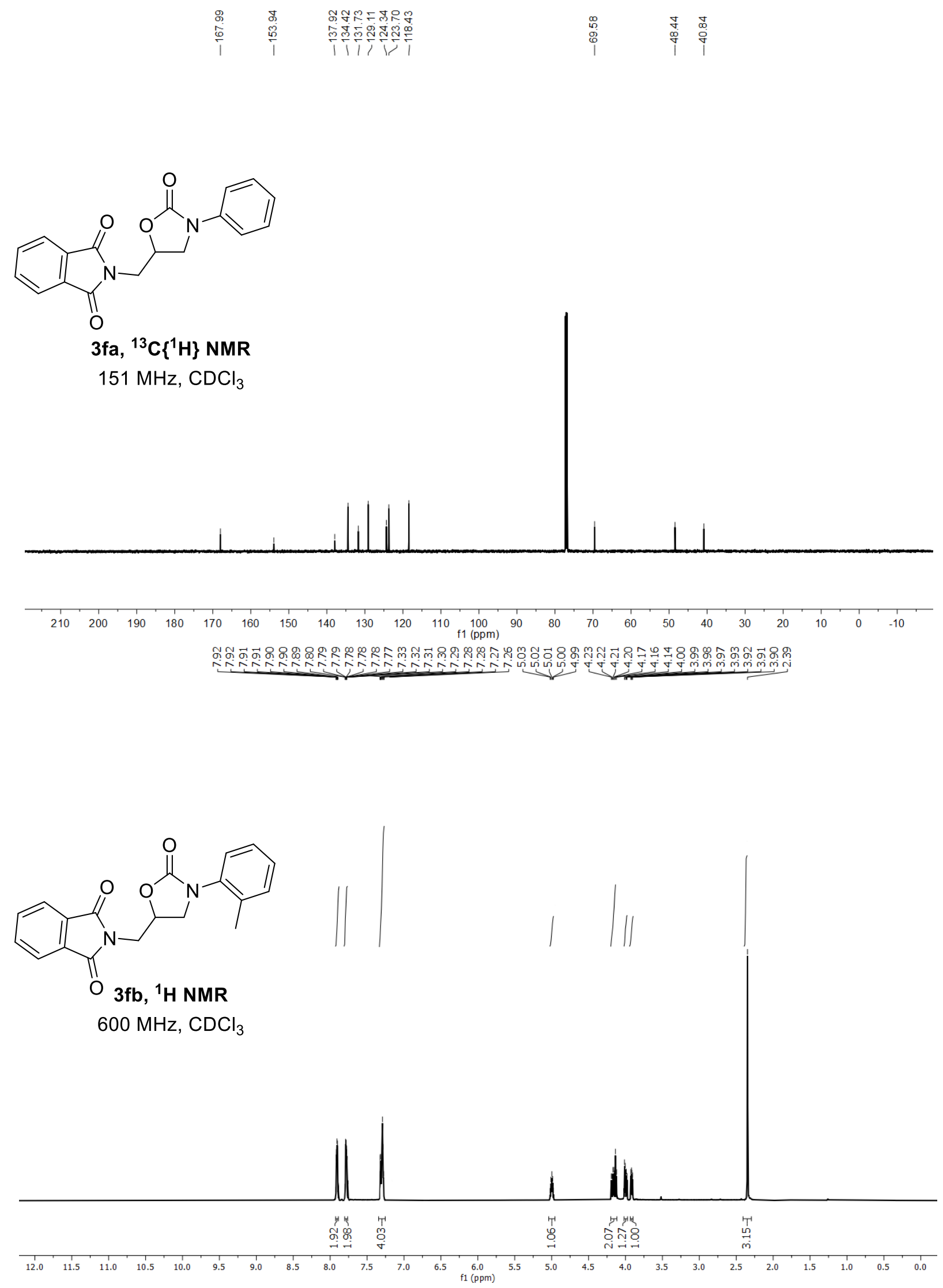


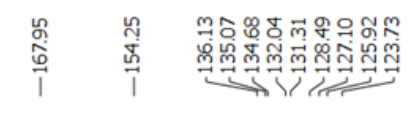

早 景早<smiles>Cc1ccccc1N1CC(CN2C(=O)c3ccccc3C2=O)OC1=O</smiles>
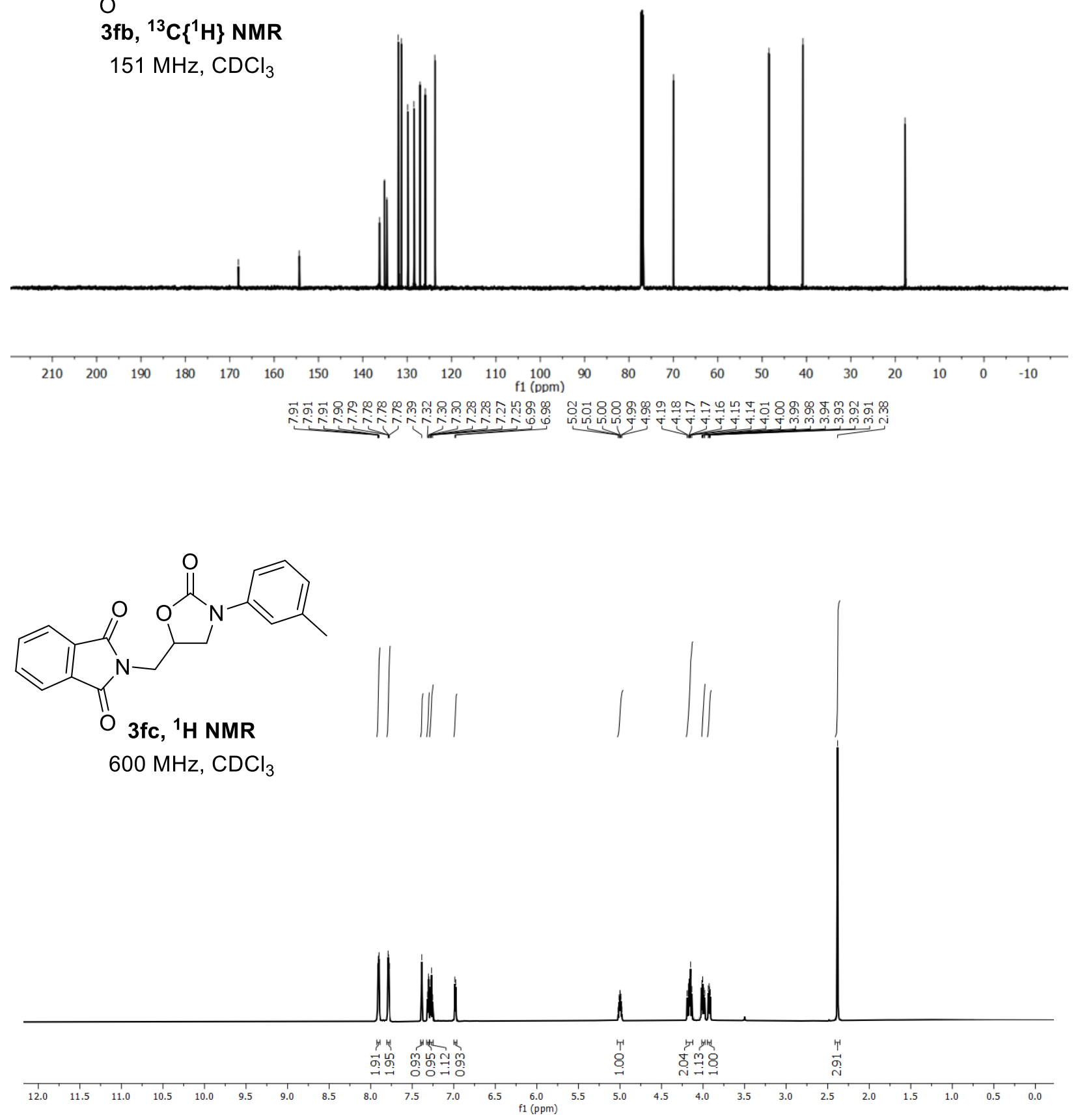

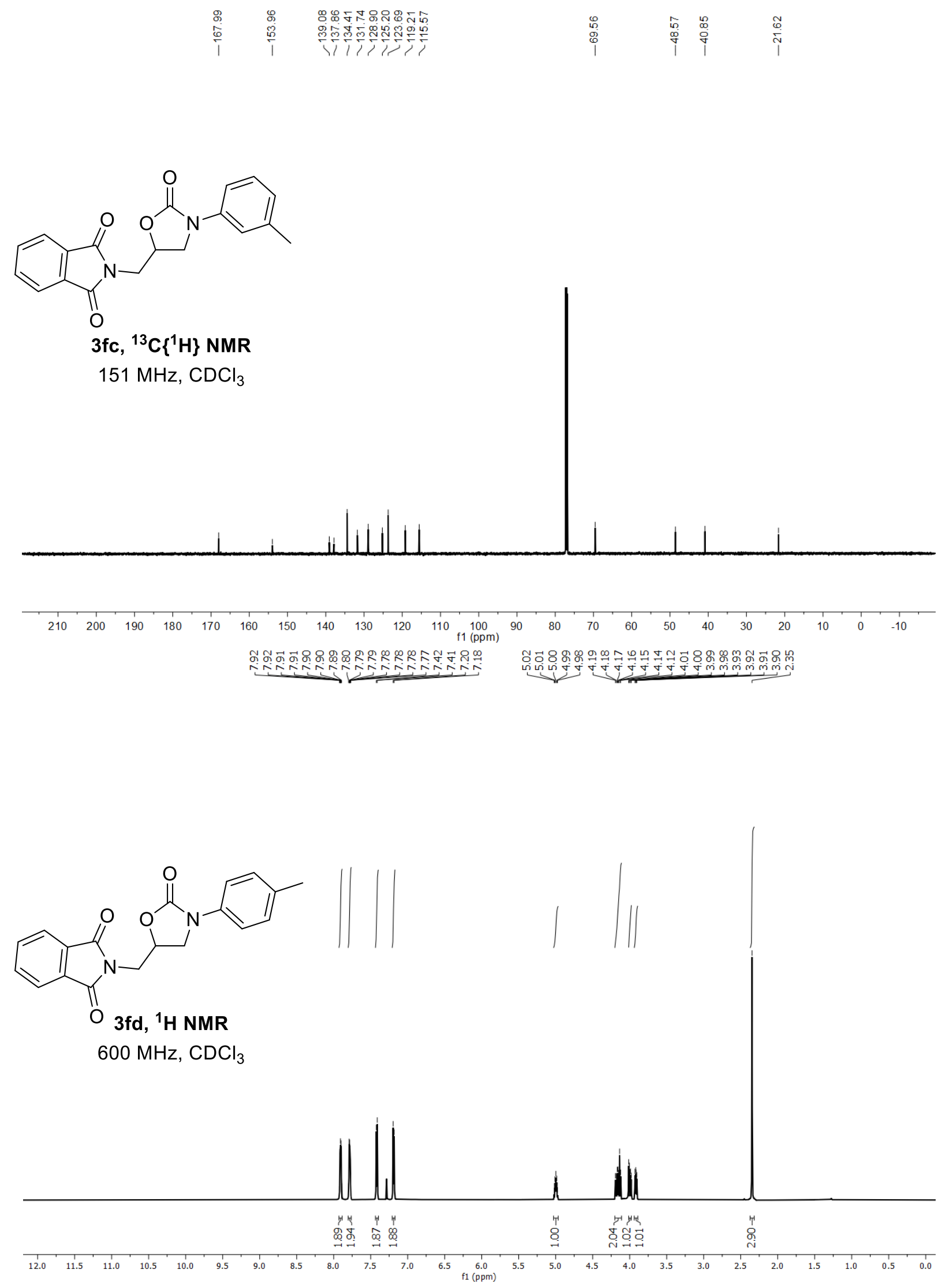

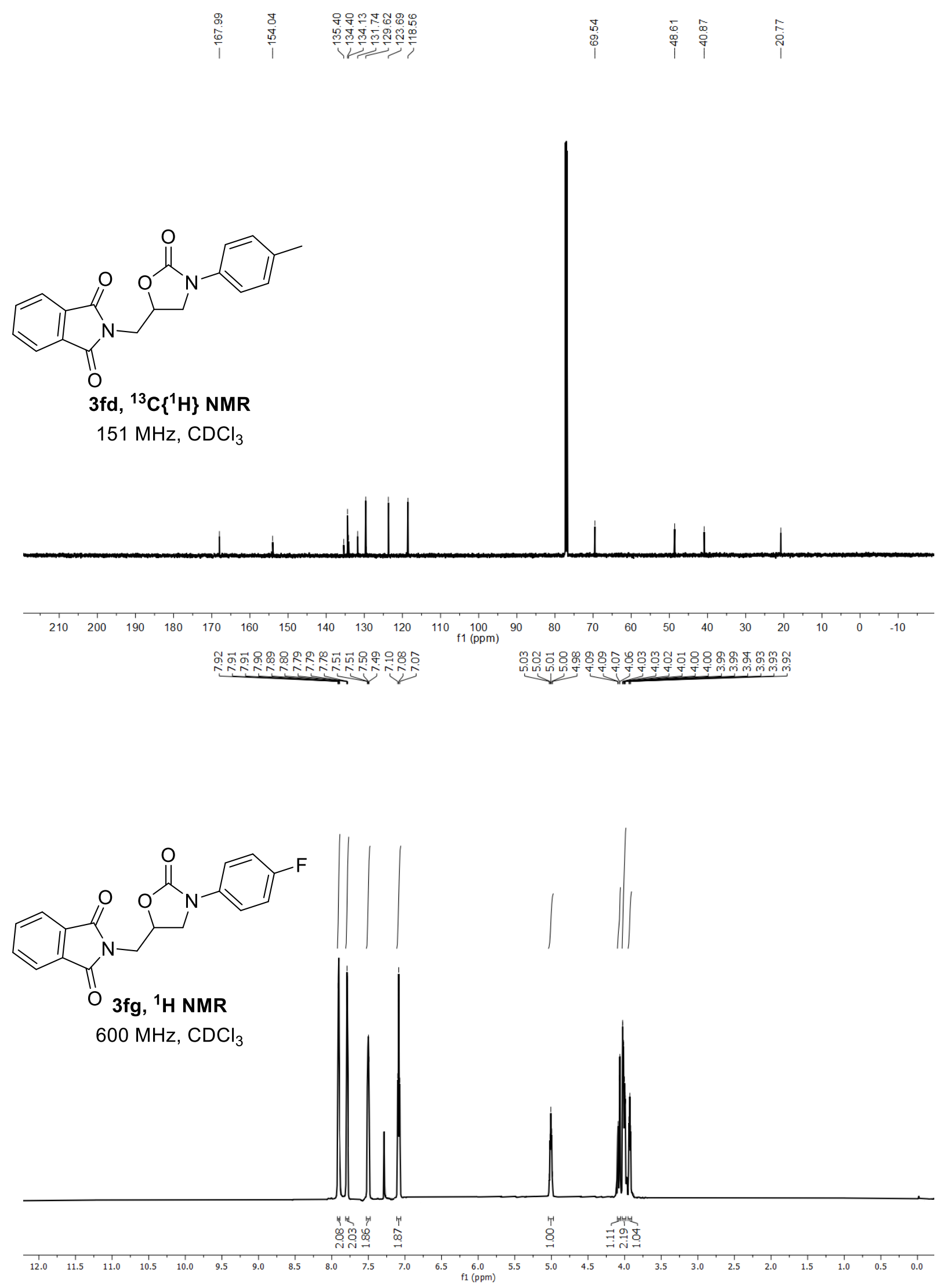

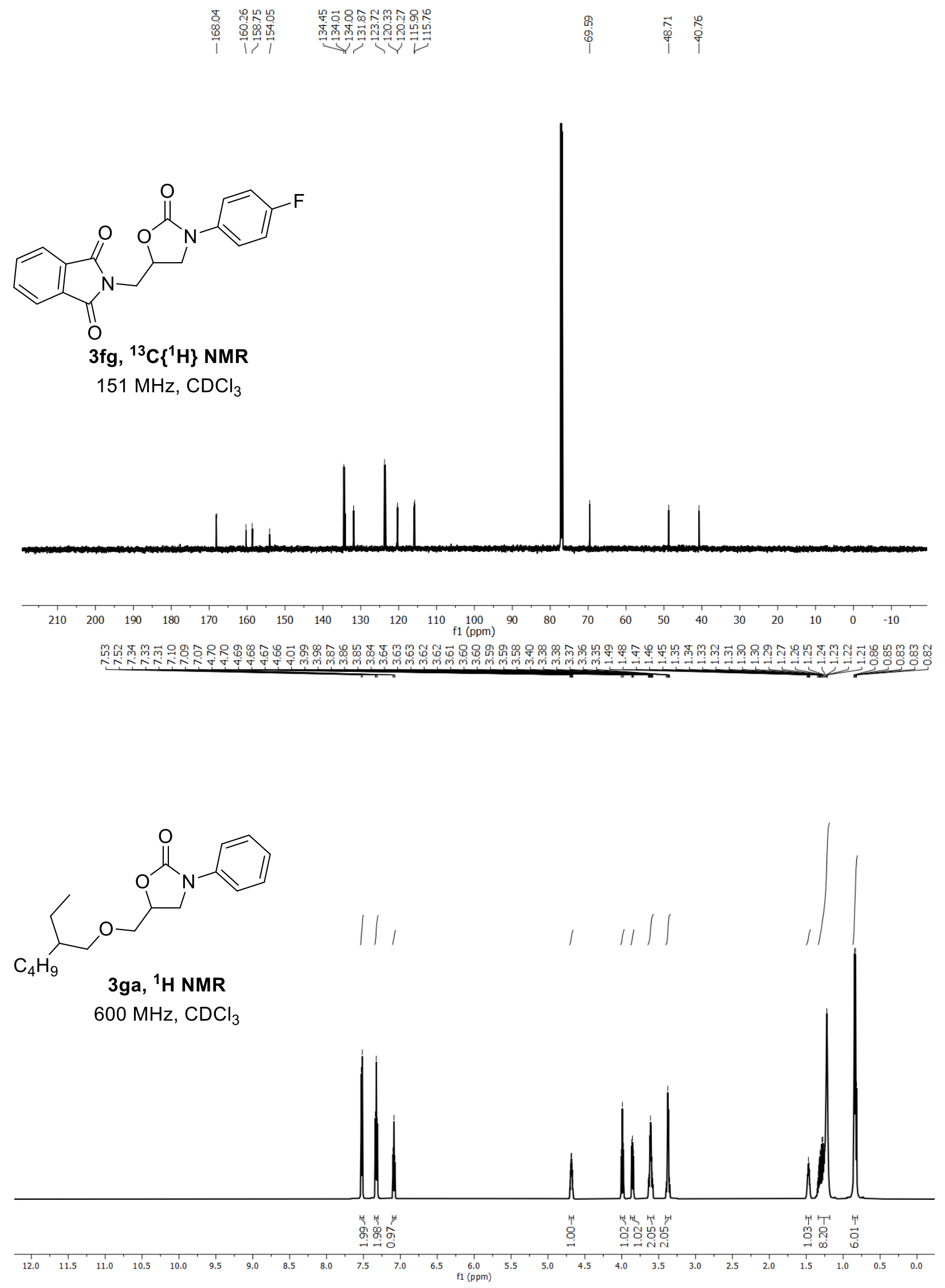

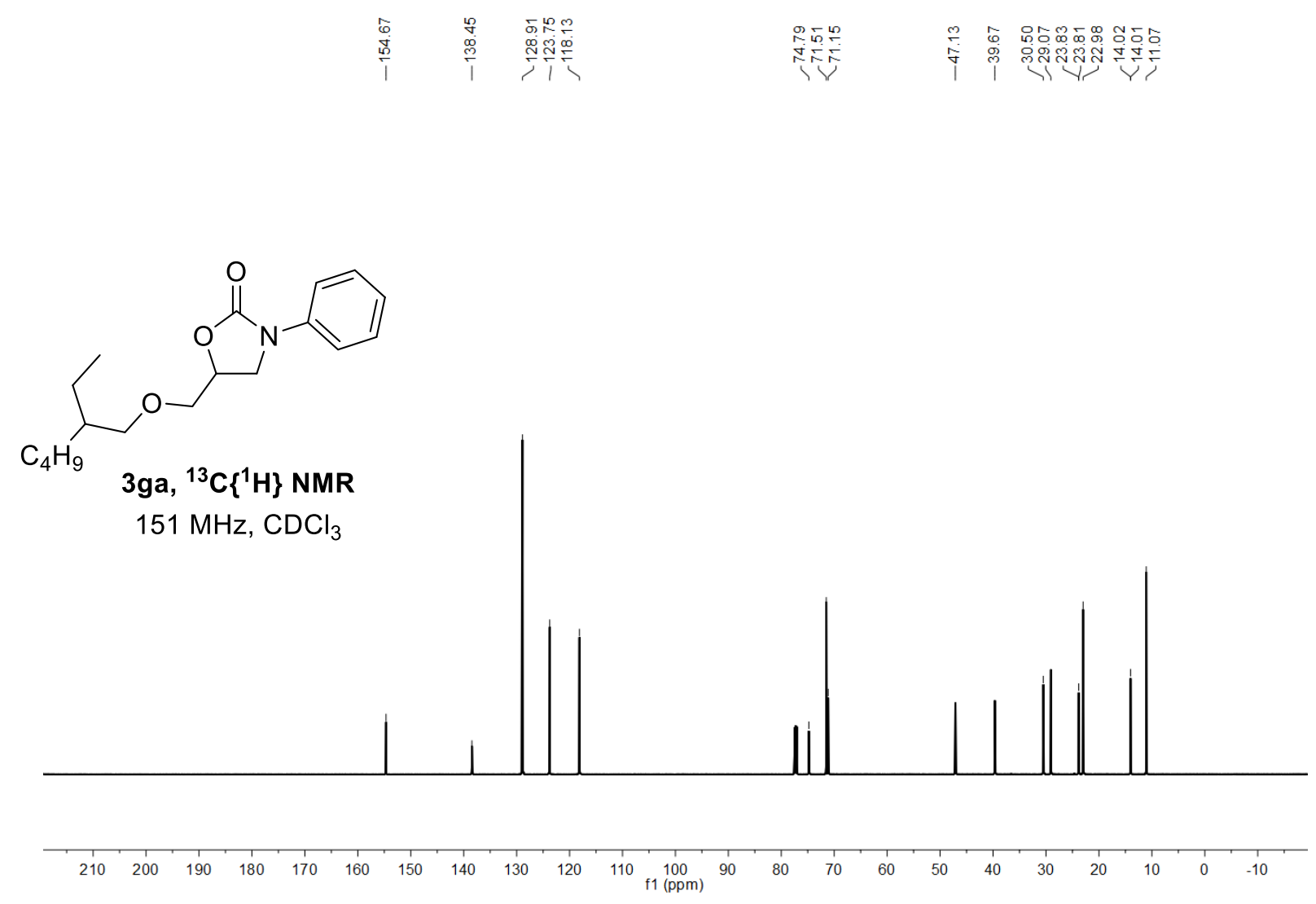

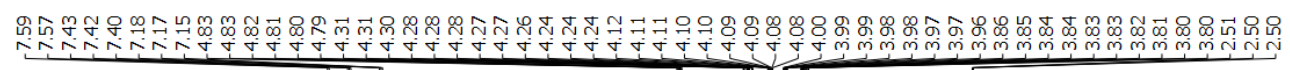

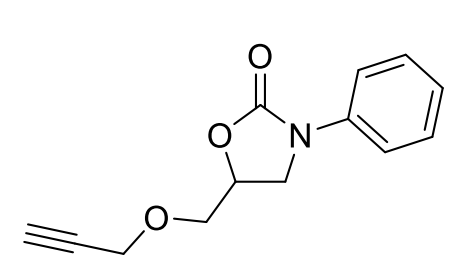

3ha, ${ }^{1} \mathrm{H}$ NMR $600 \mathrm{MHz}, \mathrm{CDCl}_{3}$

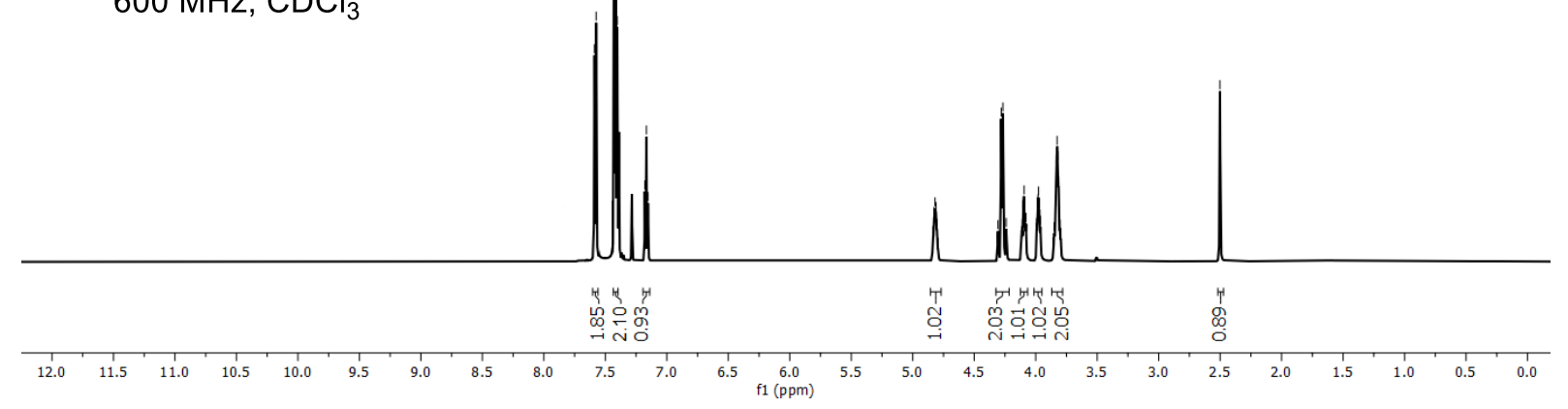



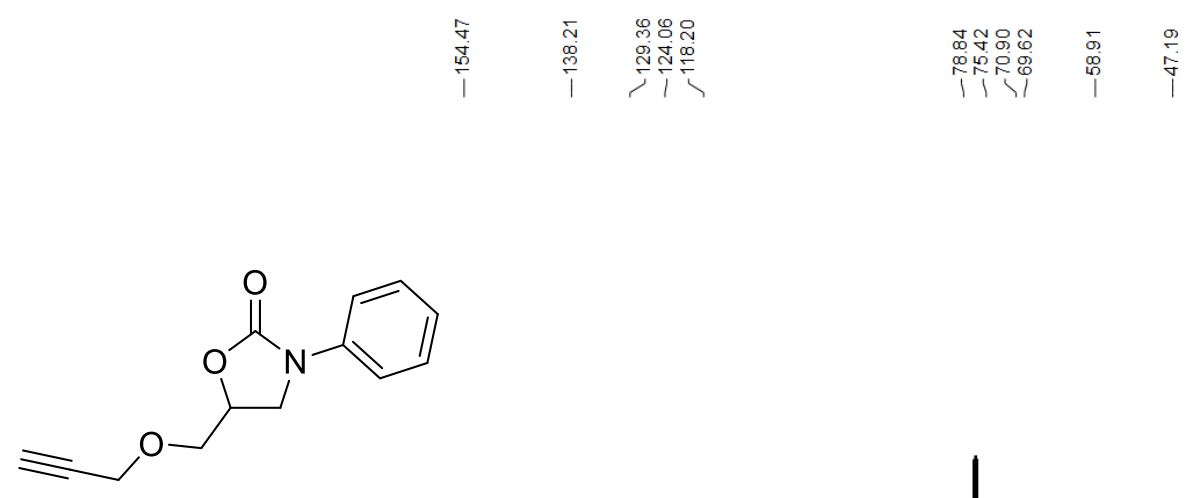

3ha, ${ }^{13} \mathrm{C}\left\{{ }^{1} \mathrm{H}\right\}$ NMR

$151 \mathrm{MHz}, \mathrm{CDCl}_{3}$
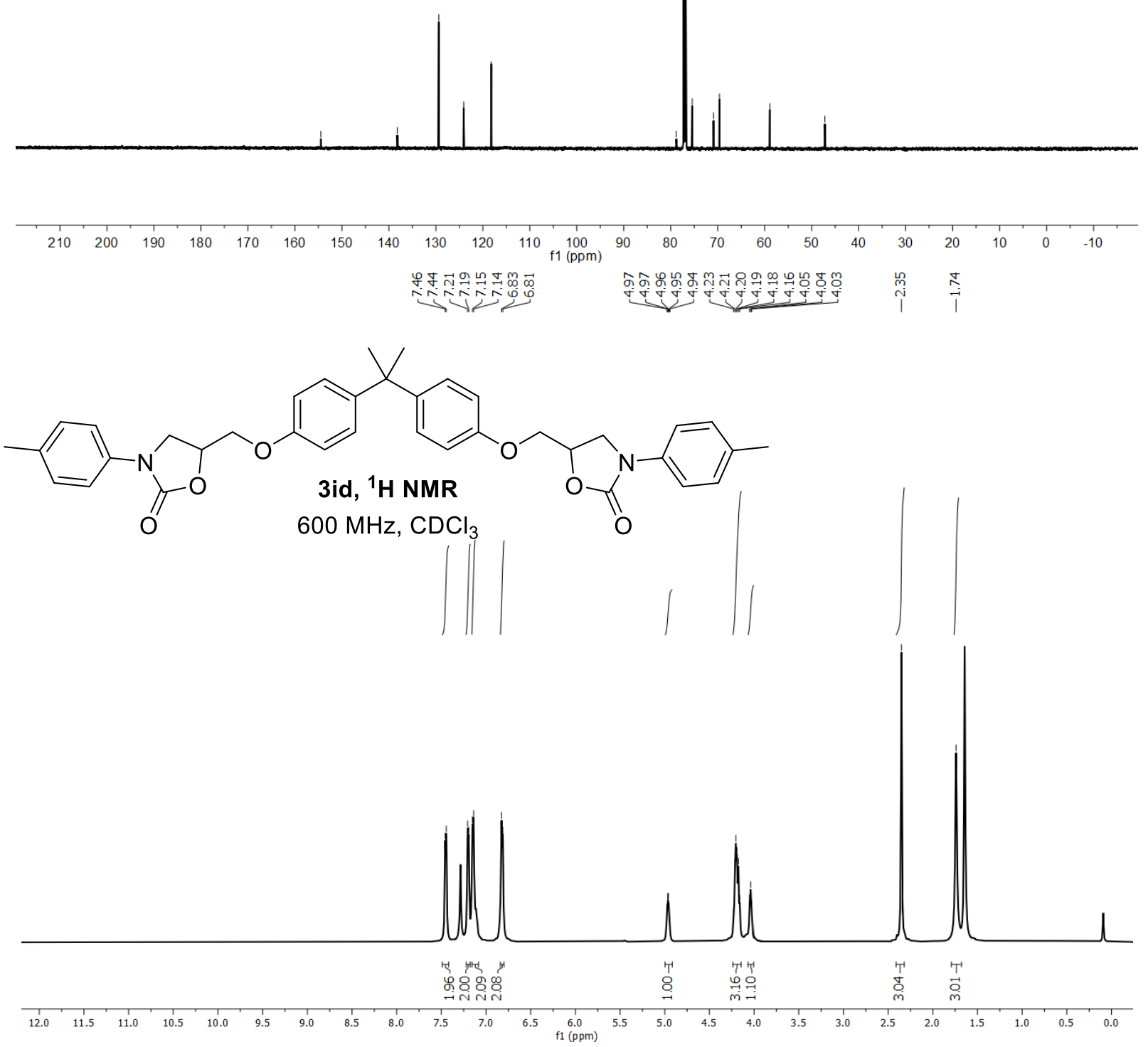

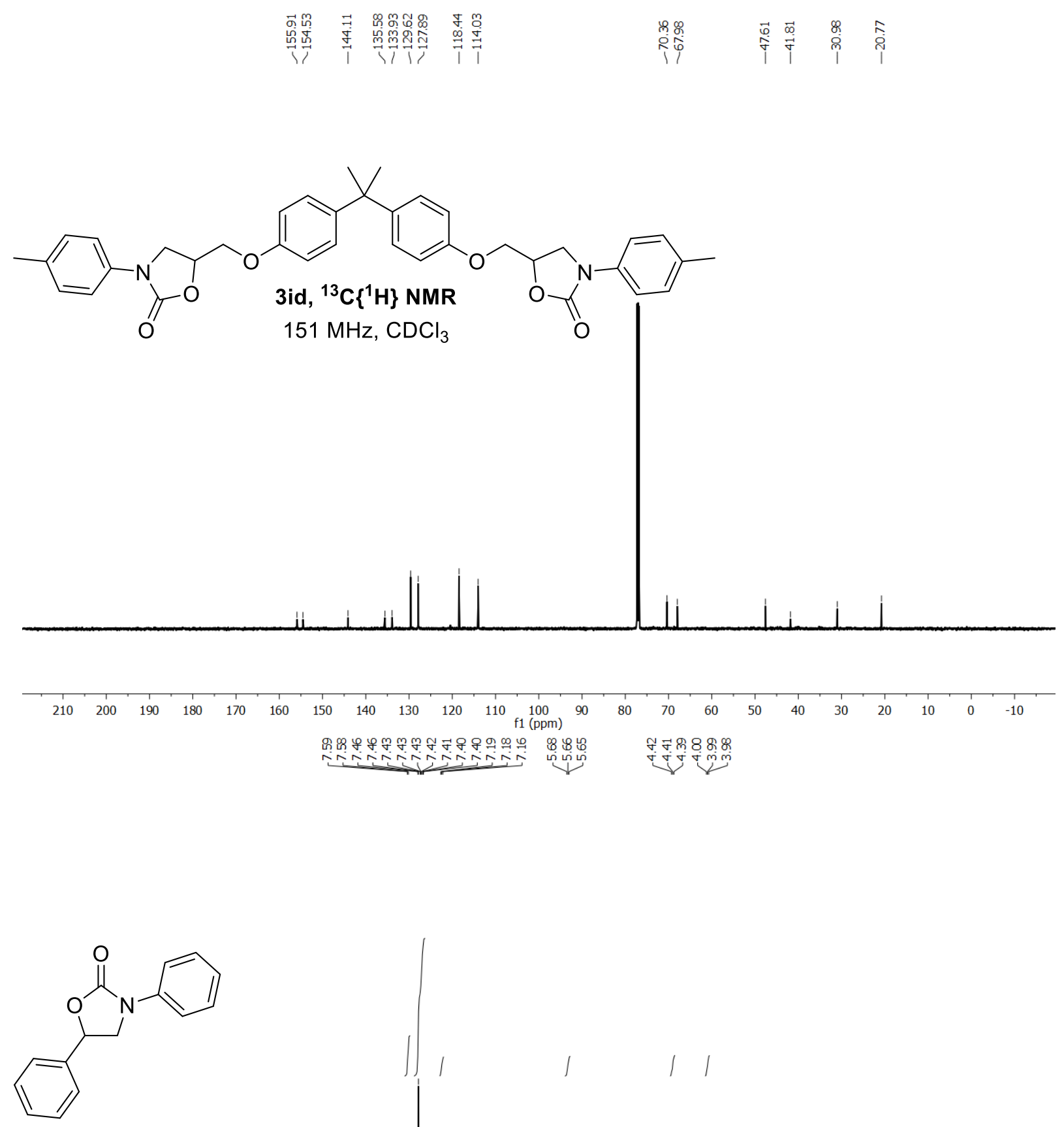

3ja, ${ }^{1} \mathrm{H}$ NMR

$600 \mathrm{MHz} \mathrm{CDCl}_{3}$

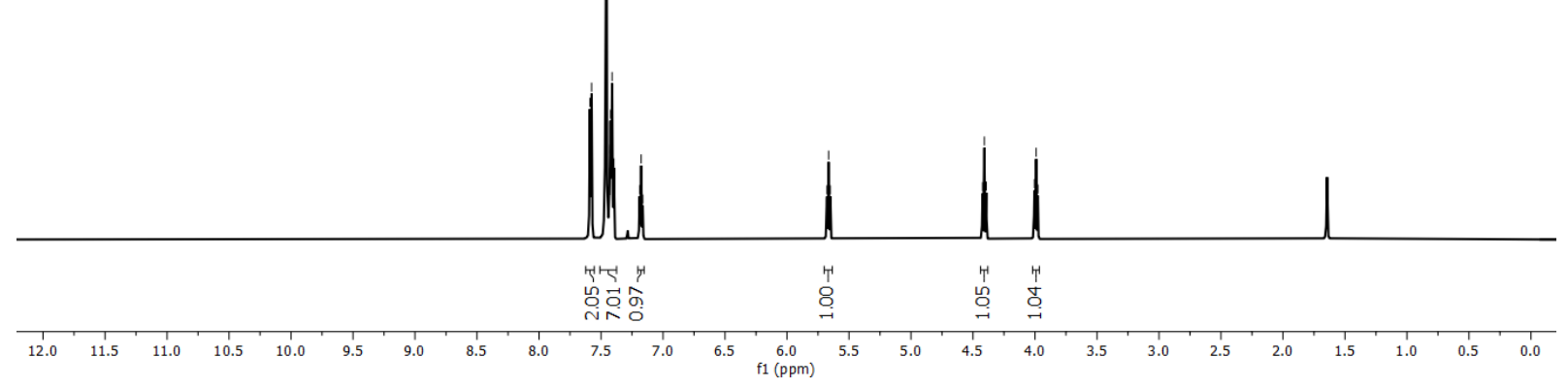




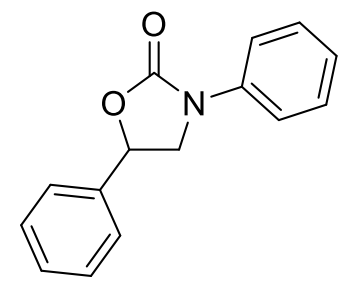

3ja, ${ }^{13} \mathrm{C}\left\{{ }^{1} \mathrm{H}\right\}$ NMR

$151 \mathrm{MHz}, \mathrm{CDCl}_{3}$
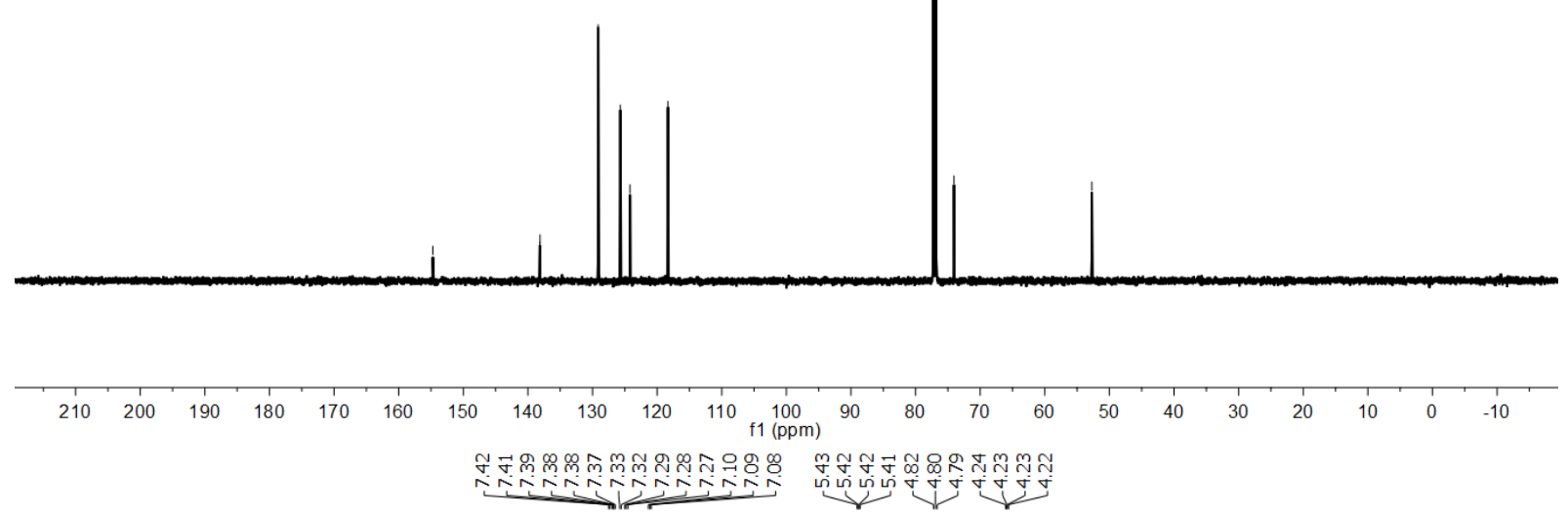<smiles>O=C1OCC(c2ccccc2)N1c1ccccc1</smiles>

4ja, ${ }^{1} \mathrm{H}$ NMR $600 \mathrm{MHz}, \mathrm{CDCl}_{3}$

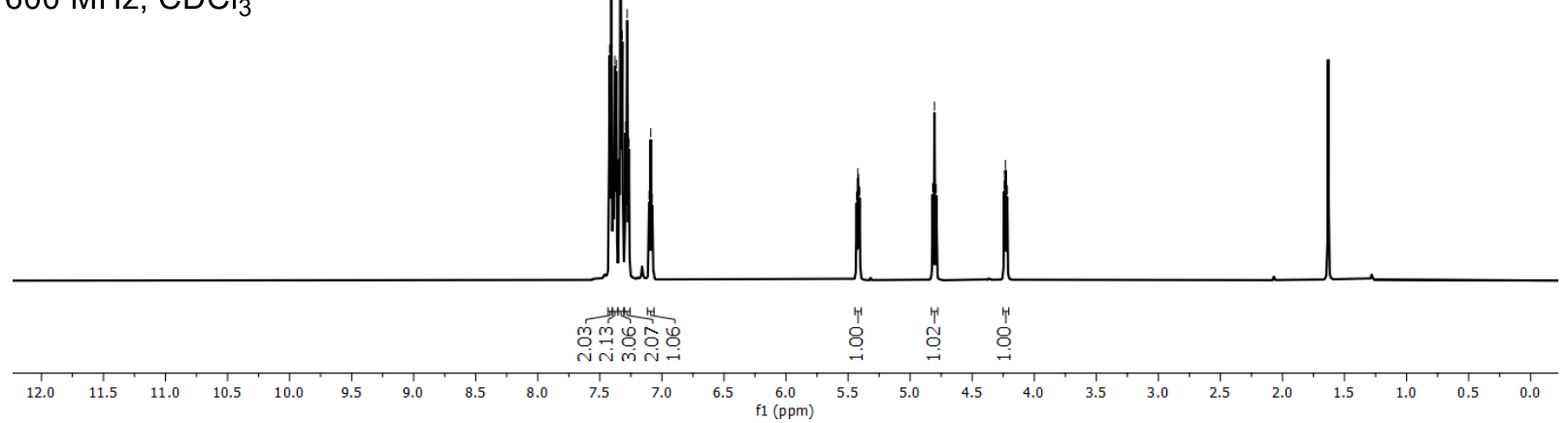




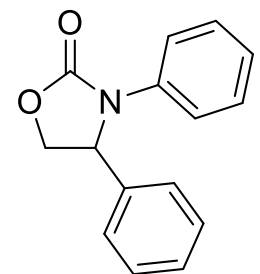

4ja, ${ }^{13} \mathrm{C}\left\{{ }^{1} \mathrm{H}\right\}$ NMR

$151 \mathrm{MHz}, \mathrm{CDCl}_{3}$
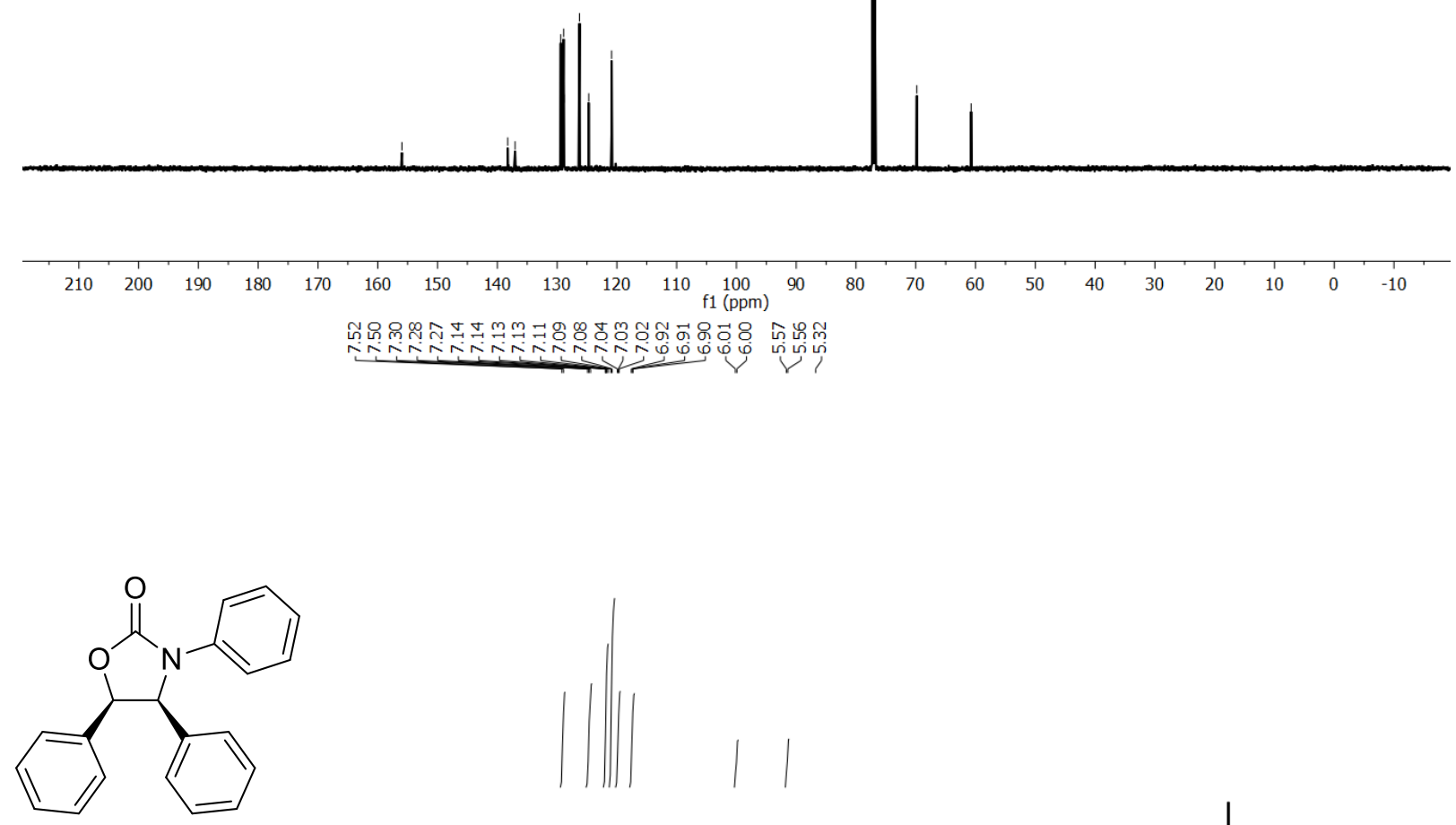

cis-3ka, ${ }^{1} \mathrm{H}$ NMR

$600 \mathrm{MHz}, \mathrm{CDCl}_{3}$

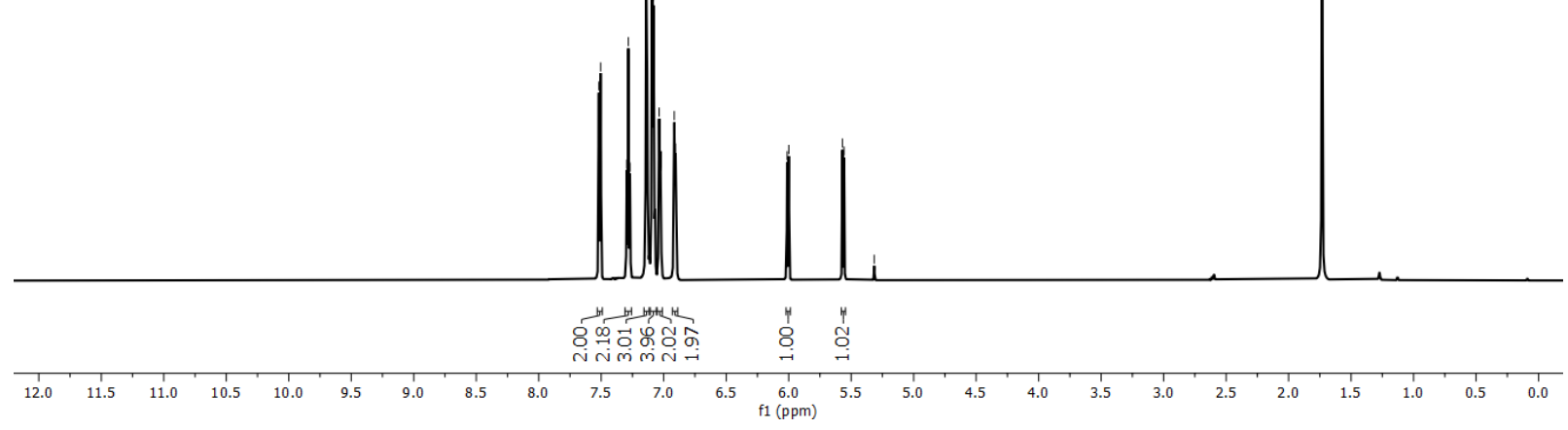



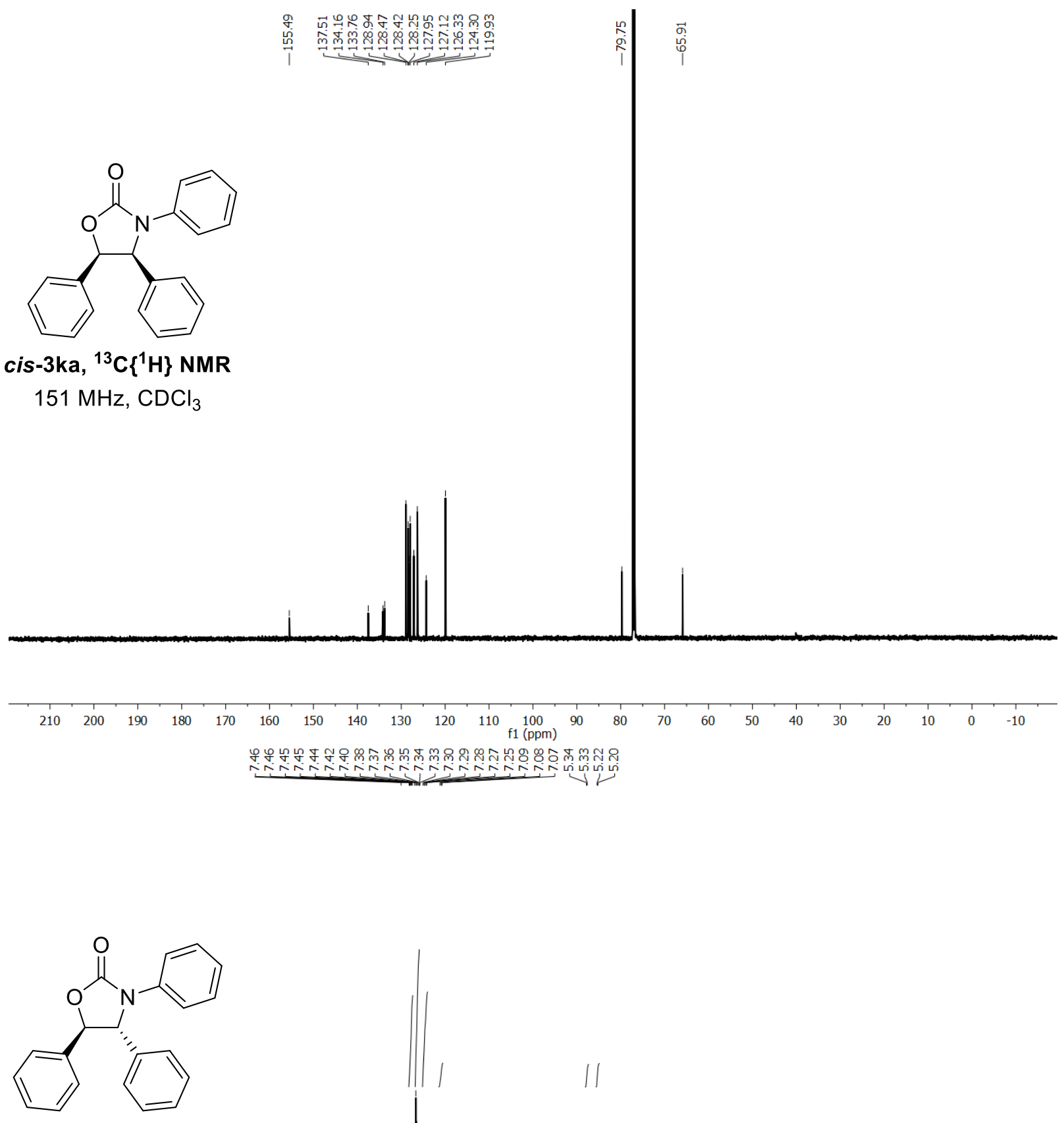

trans-3ka, ${ }^{1} \mathrm{H}$ NMR

$600 \mathrm{MHz}, \mathrm{CDCl}_{3}$

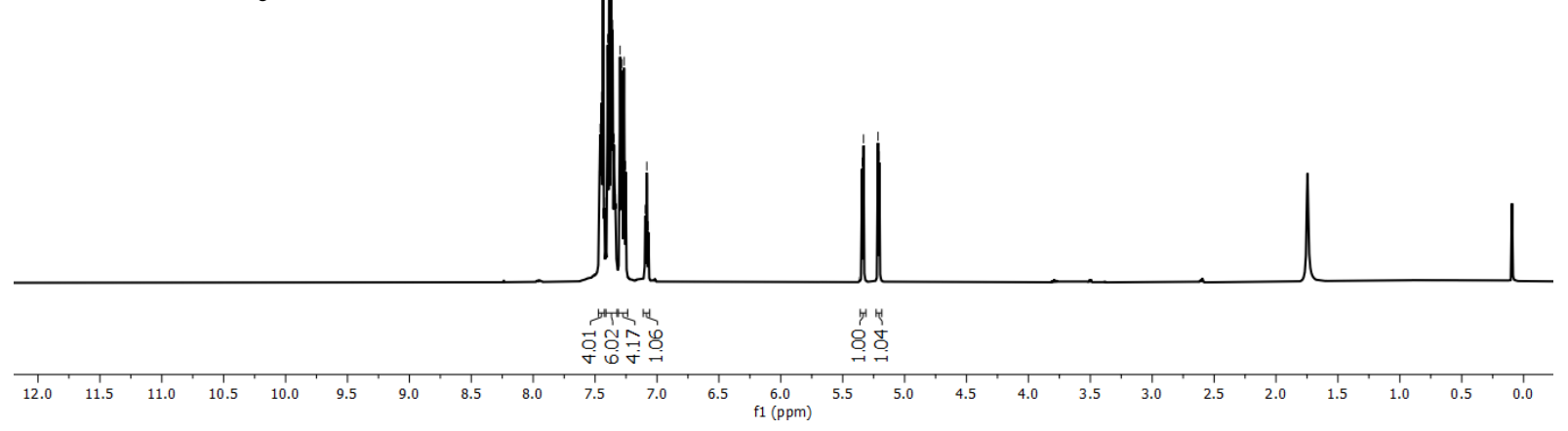




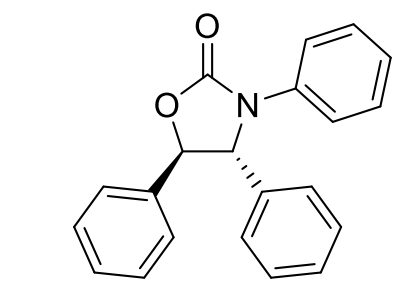

trans-3ka, ${ }^{13} \mathrm{C}\left\{{ }^{1} \mathrm{H}\right\}$ NMR

$151 \mathrm{MHz}, \mathrm{CDCl}_{3}$
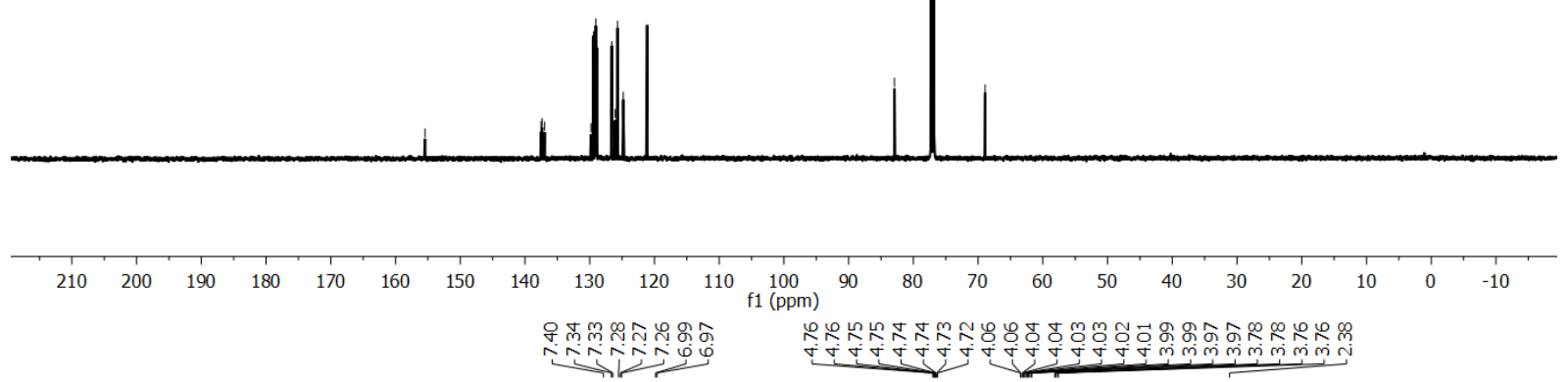<smiles>Cc1cccc(N2CC(CO)OC2=O)c1</smiles><smiles>C1=CC=C1</smiles>

3Ic, ${ }^{1} \mathrm{H}$ NMR

$600 \mathrm{MHz} \mathrm{CDCl}_{3}$

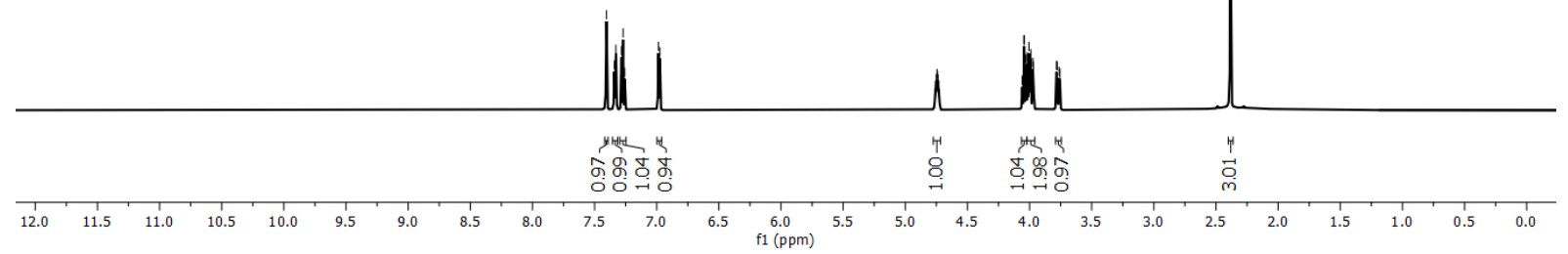




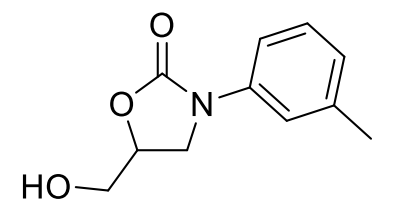

3lc, ${ }^{13} \mathrm{C}\left\{{ }^{1} \mathrm{H}\right\}$ NMR

$151 \mathrm{MHz}, \mathrm{CDCl}_{3}$
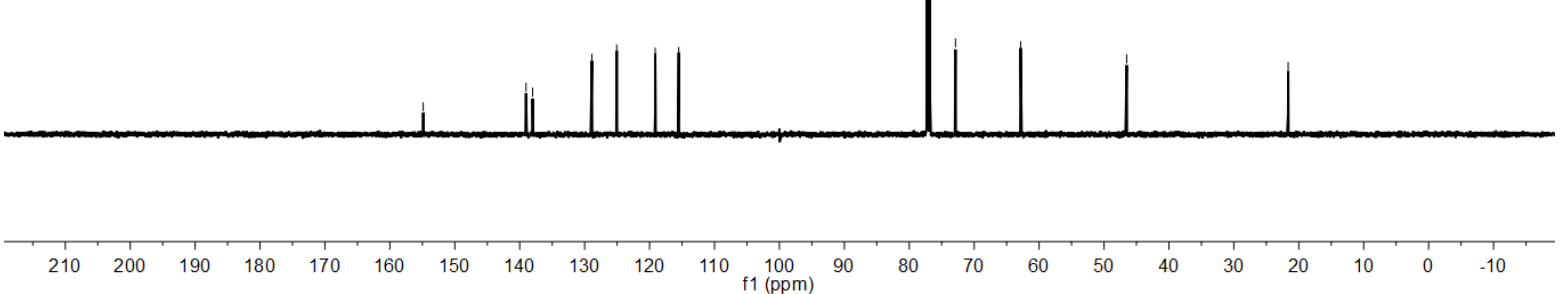

HPLC spectrum and data for $3 \mathrm{ea}$

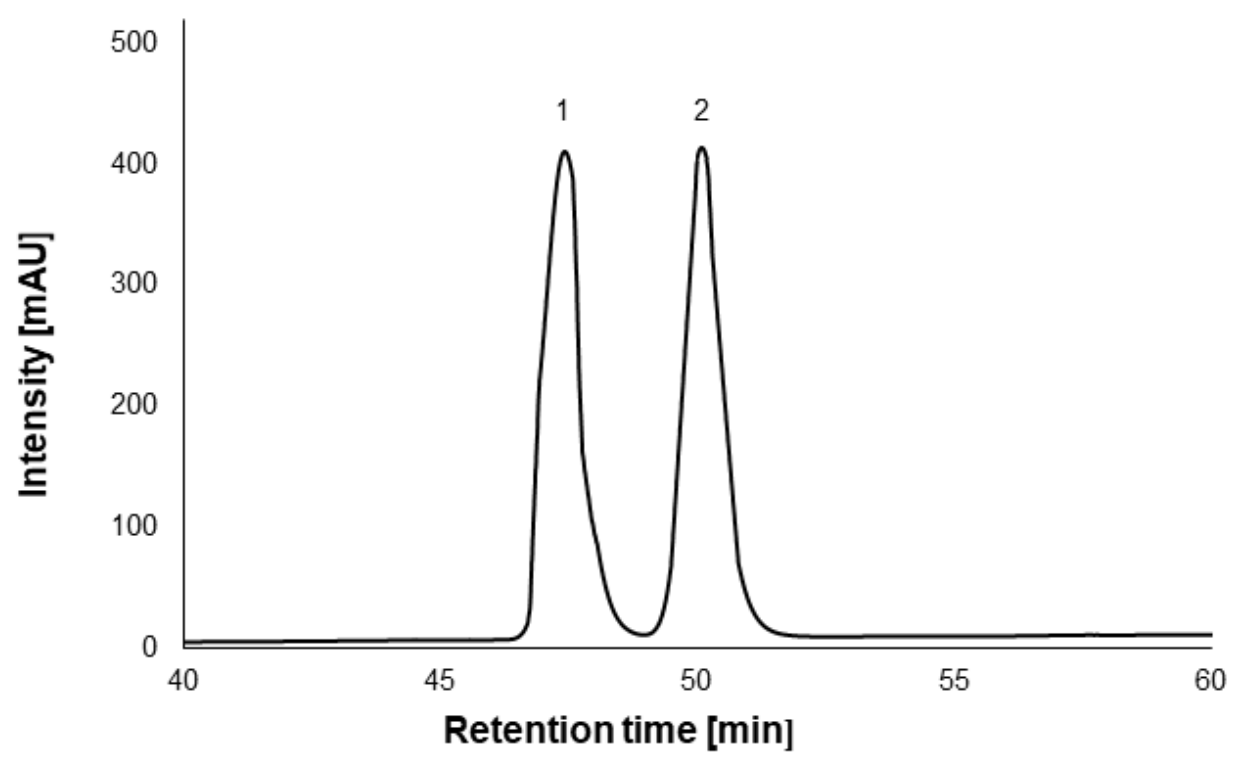

\begin{tabular}{|c|c|c|c|c|}
\hline Peak number & Retention time (min) & Area & height & Area\% \\
\hline 1 & 47.81 & 16952.1266 & 421.35698 & 49.92 \\
\hline 2 & 50.09 & 17008.8657 & 423.16598 & 50.08 \\
\hline
\end{tabular}


HPLC spectrum and data for (S)-3ea

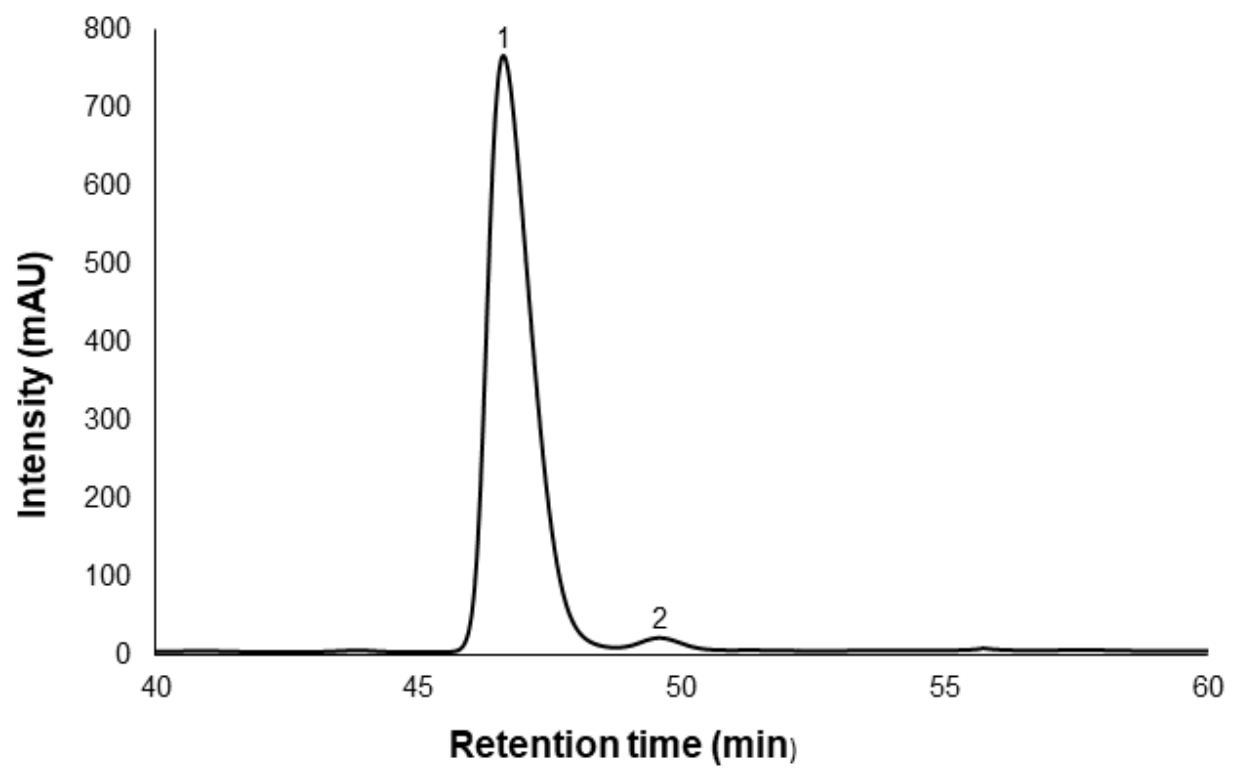

\begin{tabular}{|c|c|c|c|c|}
\hline Peak number & Retention time (min) & Area & height & Area\% \\
\hline 1 & 47.11 & 31873.1896 & 786.12382 & 99.2 \\
\hline 2 & 49.94 & 254.98552 & 53.87935 & 0.08 \\
\hline
\end{tabular}

\section{HPLC spectrum and data for $(R)$-3ea}

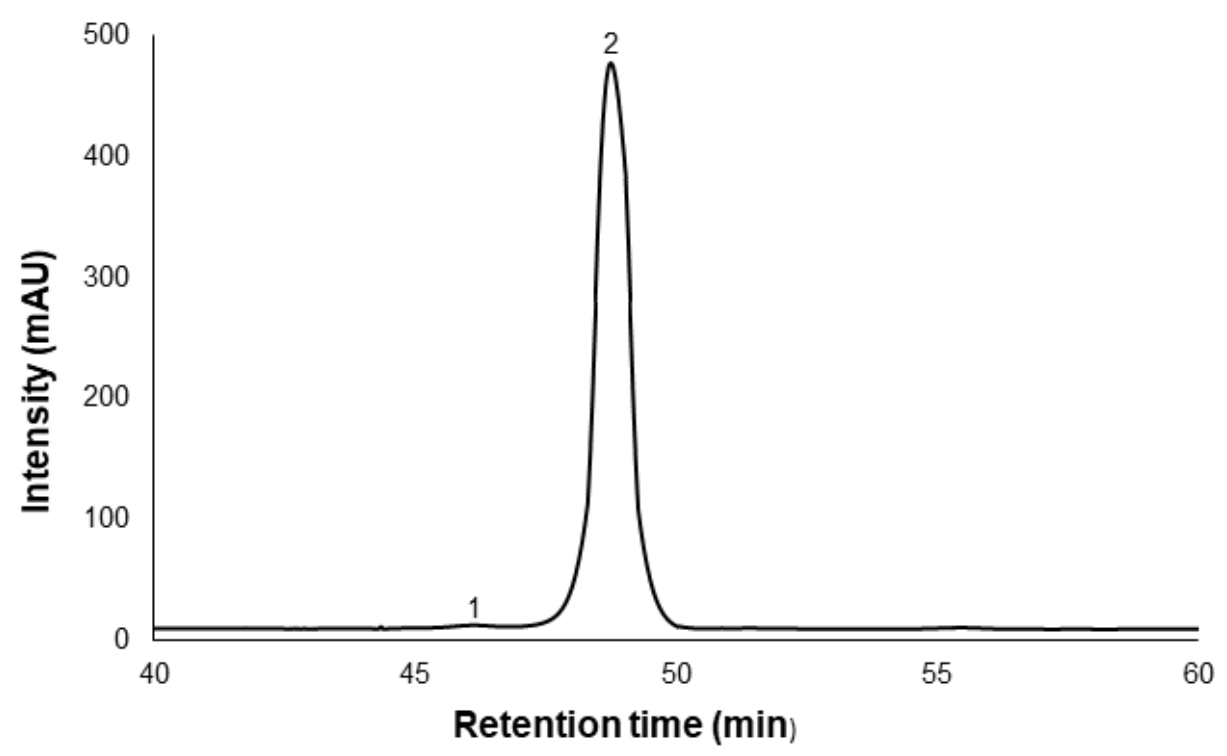

\begin{tabular}{|c|c|c|c|c|}
\hline Peak number & Retention time (min) & Area & height & Area\% \\
\hline 1 & 46.91 & 192.20814 & 4.56834 & 0.9 \\
\hline 2 & 49.03 & 20673.3257 & 491.35682 & 99.1 \\
\hline
\end{tabular}

